Review

\title{
Functionalization Strategies of PEDOT and PEDOT:PSS Films for Organic Bioelectronics Applications
}

\author{
Gonzalo E. Fenoy ${ }^{1}(0)$, Omar Azzaroni ${ }^{1,2, *}$, Wolfgang Knoll ${ }^{3,4}\left(\right.$ and Waldemar A. Marmisollé ${ }^{1, *}$ \\ 1 Instituto de Investigaciones Fisicoquímicas Teóricas y Aplicadas (INIFTA), Departamento de Química, \\ Facultad de Ciencias Exactas, Universidad Nacional de La Plata (UNLP), CONICET. 64 and 113, \\ La Plata 1900, Argentina; fenoygonzalo@gmail.com \\ 2 CEST-UNLP Partner Lab for Bioelectronics (INIFTA), Diagonal 64 y 113, La Plata 1900, Argentina \\ 3 AIT Austrian Institute of Technology-Biosensor Technologies, 3430 Tulln, Austria; Wolfgang.Knoll@ait.ac.at \\ 4 Department of Scientific Coordination and Management, Danube Private University, 3500 Krems, Austria \\ * Correspondence: azzaroni@inifta.unlp.edu.ar (O.A.); wmarmi@inifta.unlp.edu.ar (W.A.M.)
}

Citation: Fenoy, G.E.; Azzaroni, O.; Knoll, W.; Marmisollé, W.A.

Functionalization Strategies of PEDOT and PEDOT:PSS Films for Organic Bioelectronics Applications. Chemosensors 2021, 9, 212.

https://doi.org/10.3390/

chemosensors 9080212

Academic Editor: Boris Lakard

Received: 29 June 2021

Accepted: 4 August 2021

Published: 6 August 2021

Publisher's Note: MDPI stays neutral with regard to jurisdictional claims in published maps and institutional affiliations.

Copyright: (c) 2021 by the authors. Licensee MDPI, Basel, Switzerland. This article is an open access article distributed under the terms and conditions of the Creative Commons Attribution (CC BY) license (https:// creativecommons.org/licenses/by/ $4.0 /)$.

\begin{abstract}
Organic bioelectronics involves the connection of organic semiconductors with living organisms, organs, tissues, cells, membranes, proteins, and even small molecules. In recent years, this field has received great interest due to the development of all kinds of devices architectures, enabling the detection of several relevant biomarkers, the stimulation and sensing of cells and tissues, and the recording of electrophysiological signals, among others. In this review, we discuss recent functionalization approaches for PEDOT and PEDOT:PSS films with the aim of integrating biomolecules for the fabrication of bioelectronics platforms. As the choice of the strategy is determined by the conducting polymer synthesis method, initially PEDOT and PEDOT:PSS films preparation methods are presented. Later, a wide variety of PEDOT functionalization approaches are discussed, together with bioconjugation techniques to develop efficient organic-biological interfaces. Finally, and by making use of these approaches, the fabrication of different platforms towards organic bioelectronics devices is reviewed.
\end{abstract}

Keywords: functionalization; organic bioelectronics; organic electrochemical transistors; PEDOT; PEDOT:PSS; bioconjugation; biosensing

\section{Introduction}

Organic bioelectronics comprises the association of organic semiconductors with biological entities of all orders of organization i.e., living organisms, organs, tissues, cells, membranes, proteins, and even small molecules [1]. In the course of the last 20 years, this area has experienced great advances through the design and fabrication of original materials, innovation in fabrication technologies, and the better comprehension of the transport properties [2]. This has fueled the development of a wide range of interesting devices, such as soft actuators [3], biosensors [4], recording probes [5], and organic electrochemical transistors (OECTs) [6,7], enabling the detection of several relevant biomarkers, the stimulation and sensing of cells and tissues and the recording of electrophysiological signals [8,9].

In this regard, conducting polymers (CPs) are the quintessential material for the fabrication of organic bioelectronics devices due to their organic nature and semiconducting features [10,11]. Moreover, CPs can be seen as organic mixed ionic-electronic conductors, since changes in CPs conductivity through redox commutation are accompanied by ion transport to maintain charge balance [12]. This ionic-electronic coupling together with the soft nature of these materials have propelled its use in organic bioelectronics, yielding the development of flexible organic conducting devices which can interface very efficiently with biological entities $[10,13]$. 
However, some CPs can present some drawbacks when considering their interfacing with biomolecules, and during their interaction with living cells or tissues. This is the case of polyaniline (PANI), which presents poor conductivity in physiological $\mathrm{pH}$ ranges, limiting its use in organic bioelectronic devices [14,15]. In this regard, poly(3,4ethylenedioxythiophene) (PEDOT) presents record-high conductivity in neutral media, as well as excellent electrochemical, thermal and moisture stability, and it can be easily prepared on a wide range of substrates [16-18]. These features have converted this $\mathrm{CP}$ in the most employed material, and several reports have been made since its appearance in this field [19].

On the other hand, when dealing with device fabrication for organic bioelectronics, the coupling of the organic semiconductors with the biological entities is not trivial and involves the most critical step towards the development of efficient devices. This issue originates from the different nature and properties of these building blocks [19]. In this respect, the effective coupling of biological units with PEDOT films becomes challenging for several reasons. On one side, the lack of functional groups in pristine PEDOT films hinders the direct incorporation of biomolecules on their surface [20]. Moreover, the integration of the biological entities in the polymerization solution involves an aggressive environment for biomolecules and denaturing for proteins, as usually organic solvents and high curing temperatures are used [21]. On the other hand, when employing PEDOT:PSS films, the acidity/toxicity [22], and the overall negative charge of the conducting polymerpolyelectrolyte complex restricts its interaction and integration with negatively charged entities, such as cells, DNA/RNA and most proteins at physiological $\mathrm{pH}$ conditions, requiring the deposited films to be further modified [23]. Therefore, the development of strategies to perform the functionalization of PEDOT and PEDOT:PSS-based surfaces with the aim of improving their biocompatibility as well as the interfacing with biomolecules is key for the developing of efficient organic bioelectronics devices.

In this review, we will focus on PEDOT and PEDOT-PSS functionalization methods towards the application of these materials in organic bioelectronics. We will first present a brief discussion of PEDOT and PEDOT:PSS films production methods, and how the features of the films can be tuned by selecting appropriate fabrication condition. Then, based on these production approaches, several strategies for the functionalization of the films will be reviewed. Finally, illustrative examples of organic bioelectronics devices based on these functionalized materials will be presented (Scheme 1).

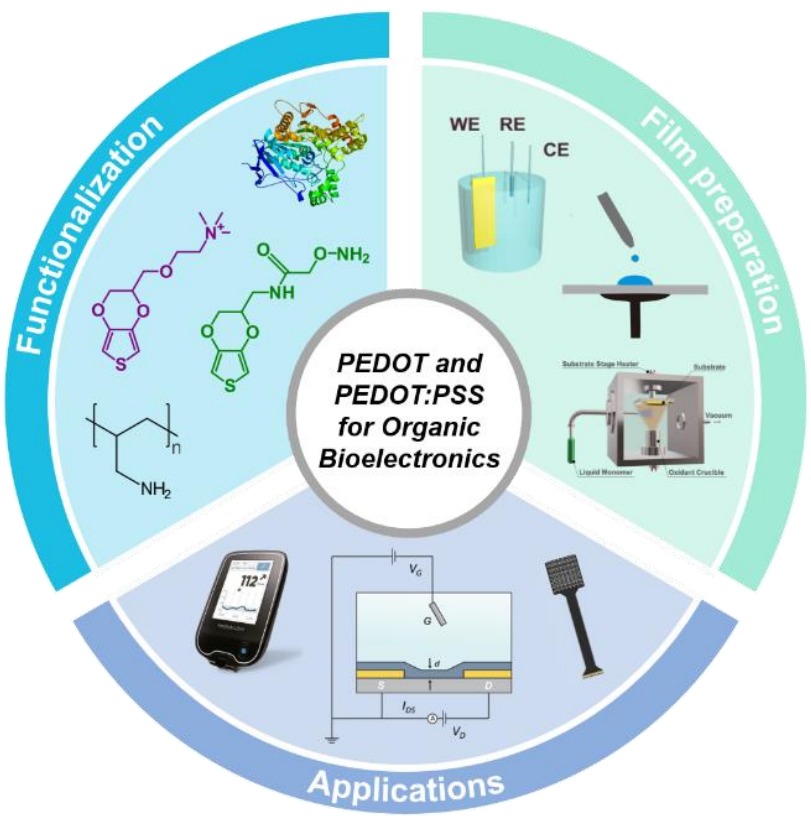

Scheme 1. Scheme of the different subjects covered in the review. 


\section{Preparation of PEDOT and PEDOT:PSS Films}

Since the functionalization approach to be performed will greatly depend on the synthesis strategy employed to obtain PEDOT-based materials, we will start this review discussing some strategies for the preparation of PEDOT and PEDOT:PSS films on different substrates. The techniques will be briefly explained, as well as their advantages and disadvantages and the effect that certain factors can have on the physicochemical features of the films. In this regard, the methods discussed can be easily classified in two broad groups: the first group involves in situ oxidation techniques, where the EDOT chemical or electrochemical polymerization is performed directly on the target substrate (Section 2.1), whereas the second group comprises the employing of a commercial solution of PEDOT:PSS, whose features are already dictated by the fabrication process (Section 2.2).

\subsection{Polymerization-Based Film Preparation}

\subsubsection{Solution-Cast Polymerization}

Solution-cast polymerization (sometimes also referred to as chemical in situ polymerization) is the simplest and probably the most employed method for the preparation of PEDOT films [16,24]. In a typical solution-cast polymerization procedure, the EDOT monomer and the oxidant agent are dissolved in an alcoholic solvent, and the mixture is subsequently casted on a substrate, which is finally annealed and washed. The technique is simple, does not requires any complex or expensive equipment, and it is suitable for large-scale production of films [25]. Moreover, films can be obtained on a wide variety of substrates.

It is known that the mechanism for the oxidative polymerization of PEDOT starts with the oxidation of EDOT by the oxidant (considered be the rate-limiting step of the reaction [26,27]), yielding a cation radical that dimerizes and is quickly stabilized by baseassisted deprotonation. Next, additional oxidant molecules oxidize the dimers, and the polymerization takes place as classic step polymerization. It is important to note here that since the growing PEDOT oligomeric (and polymeric) chains are also oxidized during the process (yielding a positively charged molecule), counter anions must be incorporated in order to maintain the charge balance $[28,29]$. Therefore, and based on this mechanism, a rational approach can be used to modulate the properties of the obtained films through the manipulation of the different synthesis parameters, such as monomer concentration, solvent, oxidizing agents/counterions, additives, and annealing temperature, among others.

For instance, this mechanism has encouraged the use of additives to diminish the rate of the polymerization reaction, as produced protons are known to increase the reaction rate and favors the production of dimers and trimers that cannot be further oxidized, limiting the conductivity of the films. Consequently, bases such as imidazole and pyridine are usually added to the polymerization solution, yielding films with higher conductivity [25]. Moreover, other additives such as the polymeric surfactant PEG-PPG-PEG and the high boiling point cosolvent $\mathrm{N}$-methyl-2-pyrrolidone have been reported to modify (and improve) the features of the obtained films [30,31].

When considering the oxidizing agent, its oxidation strength, pairing anion, and concentration are crucial factors that determine the properties of the obtained polymer film. In this sense, the most studied and employed oxidizing agent for PEDOT is Fe(III), paired either with chloride $\left(\mathrm{Cl}^{-}\right)$or tosylate (TOS $\left.{ }^{-}\right)$anions [24]. Between those anions, it has been reported that $\mathrm{Fe}(\mathrm{TOS})_{3}$ causes a reduction of the polymerization rate compared with $\mathrm{FeCl}_{3}$, which increases the conductivity of the obtained PEDOT films [17,29]. Moreover, other Fe salts such as iron(III) dodecylbenzenesulfonate $\left(\mathrm{FeDBSA}_{3}\right)$, iron(III) butylnaphthalenesulfonate $\left(\mathrm{FeBNSA}_{3}\right)$ and iron(III) camphorsulfonate $\left(\mathrm{FeCSA}_{3}\right)$ have recently been employed to obtain high-quality PEDOT films through the so-called self-inhibited polymerization (SIP) route (Figure 1) [32]. In this regard, the nature of the Fe(III) salt counterion influences the film mesostructure and in-plane conductivity of the prepared PEDOT films. It should be noted here that compared with electrochemical polymerization, the amount of counterions 
effectively in this technique is low, since the iron salt plays both roles as oxidizing agent as well as counterion supplier.
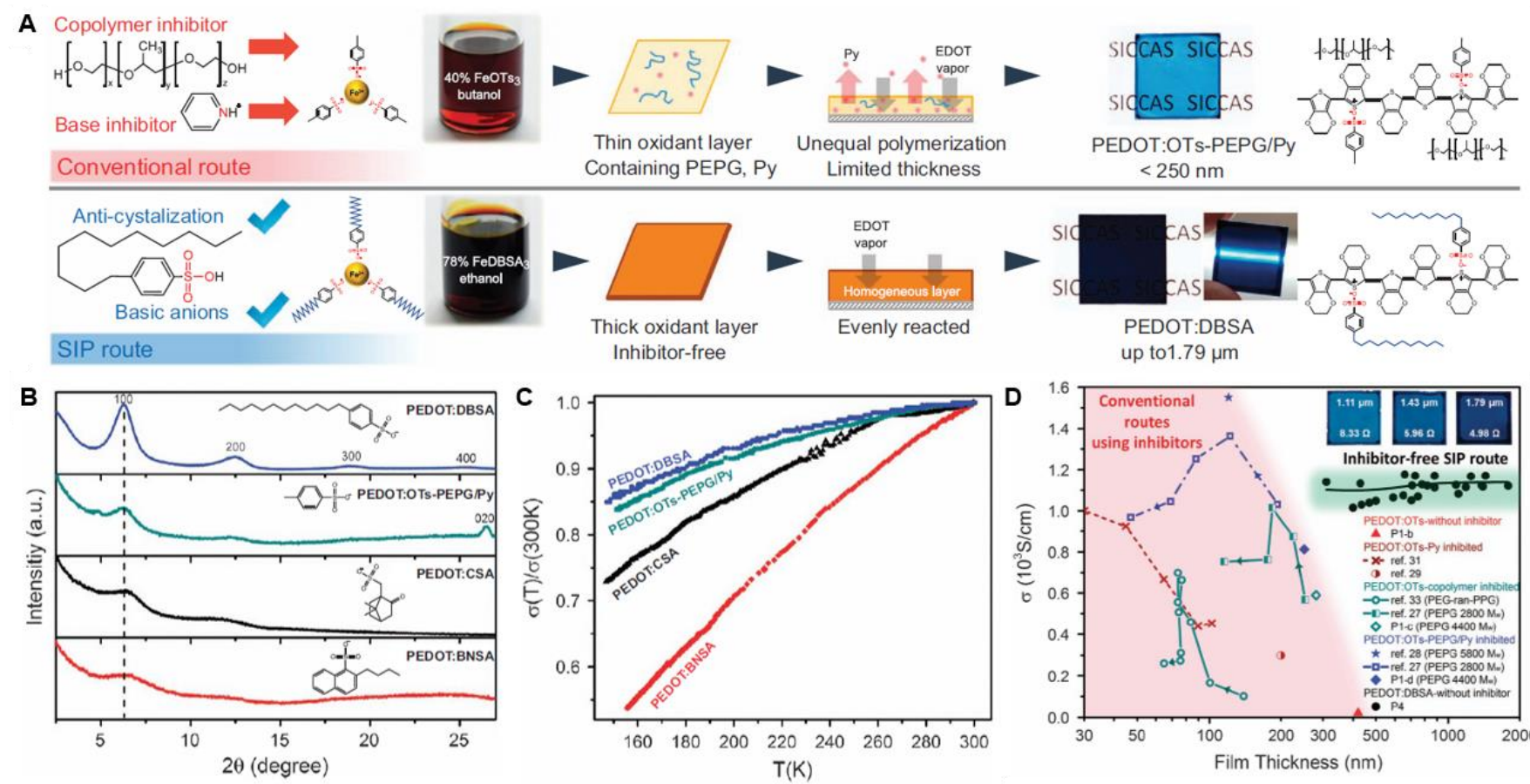
Containing PEPG, Py Limited thickness
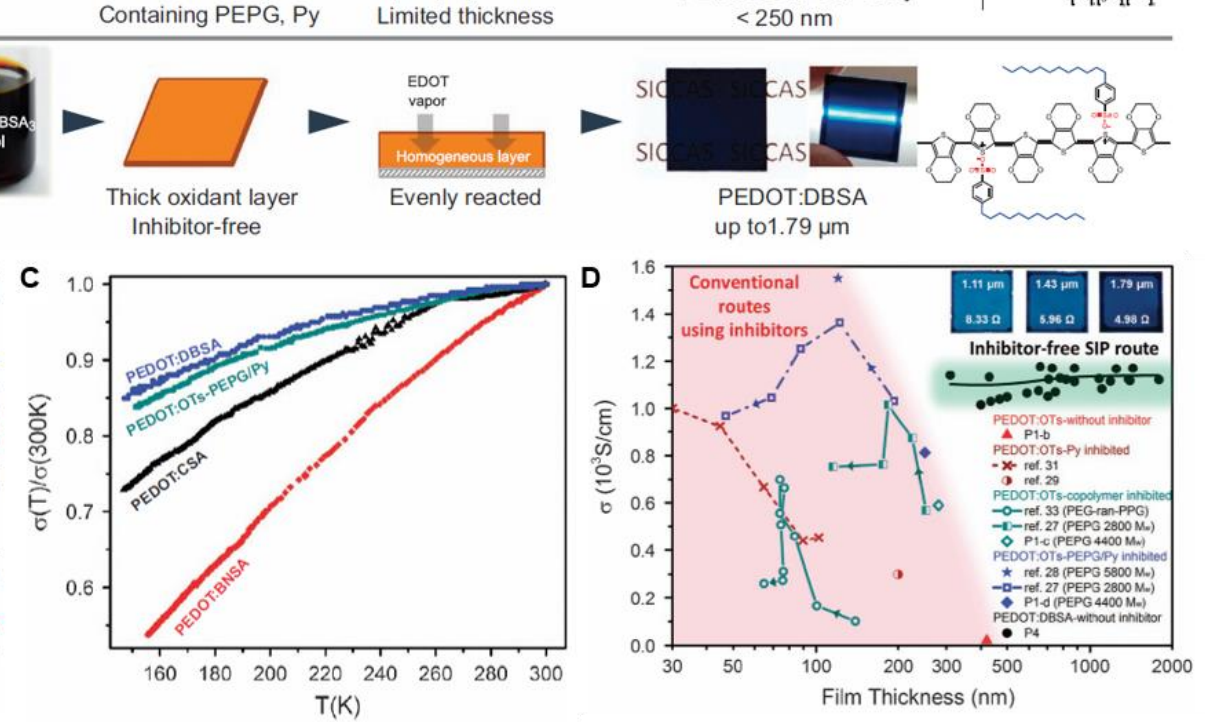

Figure 1. Scheme for the comparison of the conventional PEPG/Py-inhibited polymerization of PEDOT:TOS and the inhibitor-free self-inhibited polymerization SIP route-fabricating PEDOT:DBSA (A). Out-of-plane GIXRD diffractograms of PEDOT:DBSA, PEDOT:OTs-PEPG, PEDOT:CSA and PEDOT:BNSA (B). Temperature dependence of $\sigma$ (normalized at $300 \mathrm{~K}$ ) for PEDOT doped with anions of different steric effect (C). Film thickness dependence of $\sigma$ and comparison with other methods (D). Reproduced under the terms of the CC-BY-4.0 license [32]. Copyright 2017, The author(s).

On the other hand, the influence of the oxidant concentration and the solvent employed for the polymerization on the final features of the polymer film have also been studied. For instance, a lower oxidant concentration was shown to produce smoother films, while the thickness increases with decreasing boiling point of alcoholic solvents (while conductivity remains constant) $[28,33]$.

\subsubsection{Electrochemical Polymerization}

Although chemical oxidative polymerization makes use of chemical agents to perform the oxidation of the EDOT monomer, in electrochemical polymerization the oxidation is performed by an electrochemical technique, this being galvanostatic (constant current), potentiostatic (constant potential), galvanodynamic (pulsed current), or potentiodynamic (cyclic voltammetry or pulsed potential) [34]. In a typical electropolymerization experiment, a three-electrode cell configuration is employed (the counter, reference, and working electrodes) and the EDOT monomer is dissolved in an electrolyte solution. After the electrochemical oxidation, a doped PEDOT film attached to the working electrode surface is obtained, since the incorporation of anions from the electrolyte solution that stabilize the charged form of the polymer. Therefore, it can be easily concluded that the main drawback of this approach involves the requirement of a conductive substrate on which to deposit the film, such as Au, ITO, or glassy carbon, among others [35,36].

On the other hand, the technique also offers several advantages; for instance, as the PEDOT film is directly obtained onto the target conductive substrate, it is not necessary to employ binders for improving adhesion, and the obtained polymer films are frequently more stable than those achieved by other techniques. This intimal electrical connection between the target electrode and the polymer film is a crucial aspect for all the applications of PEDOT and, particularly, for bioelectronics [37]. In addition, the control of film thickness 
and morphology can be easily achieved by employing this technique. The ability to synthesize copolymers also constitute a very relevant advantage, and it will be further exploited when considering the polymerization of substituted EDOT-like monomers to obtain a surface prone to be biofunctionalized.

Furthermore, the use of the electrochemical approach simply allows the tuning of the features of the electropolymerized PEDOT film, since many factors such as the electrochemical method and its parameters, the working electrode nature, the electrolyte composition, and the dopants type and ratio have a significant impact on the physicochemical and electrochemical properties of the obtained polymer. For instance, the influence of the scan rate was studied by Melato et al. for films employing tetrabutylammonium perchlorate $\left(\mathrm{TBAClO}_{4}\right)$ as electrolyte, and high electropolymerization efficiency and an increase of crystallinity were observed for lower scan rates [38]. The solvent and supporting electrolyte nature also influence the morphology and electrochemical features of the obtained films. In this regard, it was reported that films prepared in propylene carbonate have a smoother structure than those prepared in acetonitrile, which leads to superior electrochromic properties (Figure 2) [39]. Other factors governing PEDOT film morphology are the electropolymerization potential, EDOT monomer concentration, type of working electrode, pre-treatment of the substrates, and current density [40-42].

\section{Cyclic Voltammetry (-1 to $1.4 \mathrm{~V}$ vs SCE)}
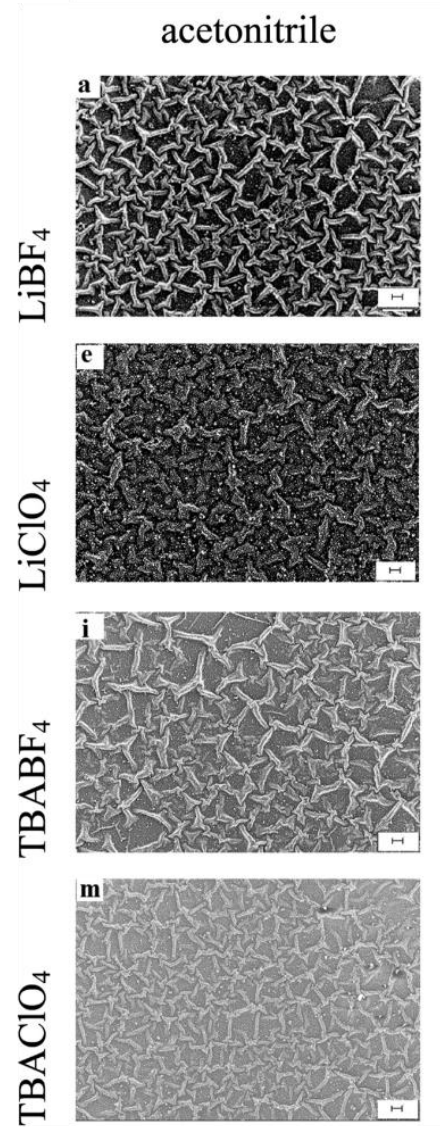

PC
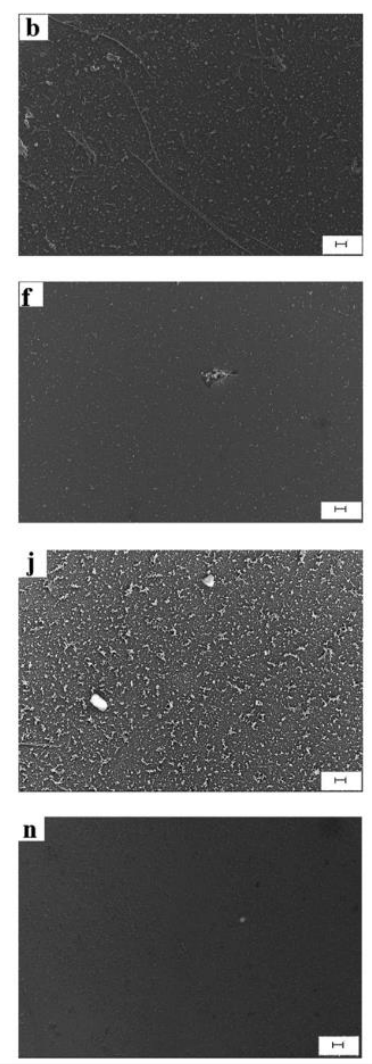

\section{Constant potential (1.2 V vs SCE)}

acetonitrile
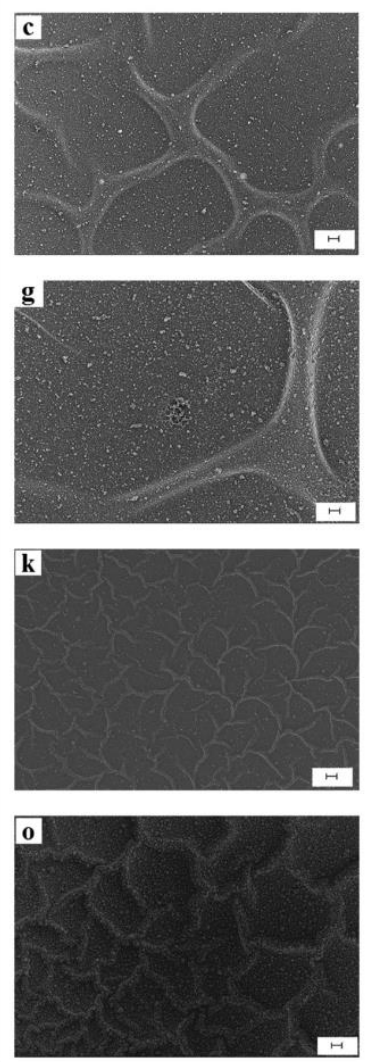

$\mathrm{PC}$
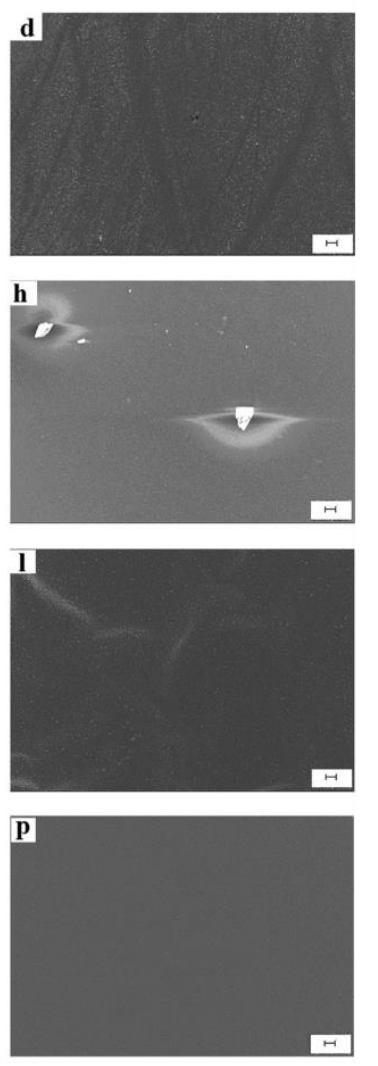

Figure 2. SEM images of PEDOT films obtained on ITO electrodes for different synthesis conditions (scale bar is $10 \mu \mathrm{m}$ ). Reprinted with permission from [39]. Copyright 2010 American Chemical Society.

When considering the dopant, as previously stated, the anion of the electrolyte becomes the counterion of the PEDOT during the polymerization, and, therefore, its choice impacts on the resulting film morphology, and mechanical and electronic features [43]. For instance, Melato et al. studied the influence of the anion and the cation during elec- 
tropolymerization of PEDOT on platinum electrodes employing different supporting electrolytes (namely $\mathrm{LiClO}_{4}, \mathrm{TBAClO}_{4}$ and $\mathrm{TBAPF}_{6}$ ) while using acetonitrile as solvent. It was shown that among all the electrolytes, the use of $\mathrm{LiClO}_{4}$ causes a higher electropolymerization efficiency and an increase of electroactivity and crystallinity of the obtained polymers. When considering the anions, $\mathrm{ClO}_{4}{ }^{-}$promoted clusters of different sizes, while the polymer film layer obtained employing $\mathrm{PF}_{6}{ }^{-}$presented a fibrillar morphology with a higher porosity. Regarding the cations, the film porosity increased when $\mathrm{Li}^{+}$was substituted by the larger cation tetrabutylammonium $\left(\mathrm{TBA}^{+}\right)$[38]. Other counterions have been also reported to be employed in PEDOT electrochemical polymerization, such as the polyanion poly(sodium 4-styrenesulfonate) (PSS) and, very recently, the sodium salt of bis(3,5-dimethyl-4-sulfonate-pyrazol-1-yl) methane (LSA) [44,45]. However, it is relevant to note that the presence bulky counteranions such as PSS causes a diminution in the conductivity of the synthesized polymer.

\subsubsection{Vapor-Phase Polymerization and Chemical Vapor Deposition}

Vapor-phase polymerization (VPP) and (polymerization through) chemical vapor deposition (CVD) are well-established approaches to obtain PEDOT films. In both techniques the polymerization process is induced chemically, requiring the use of an oxidizing agent, and the way in which this oxidant is applied involves the main difference between both techniques.

In the case of VPP, a solution containing the oxidant (and usually additives) is first casted onto the substrate, which is subsequently exposed to the EDOT monomer vapor. In the next step, the removal of the excess of reagents from the PEDOT films is performed by cleaning/rinsing the substrates. Therefore, a very simple equipment is required for this procedure, as it can be performed at lower vacuum levels or even at ambient pressure $[46,47]$. When making use of this technique, the oxidant, additives, and the process conditions (pressure, temperature, and humidity) are the main factors that can be used to modulate the final PEDOT film properties. In this sense, the most employed oxidants for the preparation of PEDOT films through VPP are $\mathrm{FeTOS}_{3}$ and $\mathrm{FeCl}_{3}$ [48]. However, other oxidizing agents have been also shown to be successful [49]. For instance, Chelawat et al. showed that PEDOT films can be obtained using bromine as oxidant, eliminating any rinsing step required with other oxidants $\left(\mathrm{as}_{\mathrm{FeCl}}\right.$ ) and making the process completely dry. Moreover, the films obtained using this oxidizing agent showed a conformal deposition together with a longer retention of the conductivity [50]. In addition, Chen et al. have recently described the VPP polymerization of PEDOT employing $\mathrm{MoCl}_{5}$ as oxidizing agent [51].

Considering the process conditions, Kim et al. showed that when employing a reaction temperature of $60^{\circ} \mathrm{C}$, highly ordered and conductive PEDOT crystalline microstructures parallel to the substrate are formed, in contrast to what happens at $20^{\circ} \mathrm{C}$ and $40{ }^{\circ} \mathrm{C}$ [46]. Humidity is another relevant factor to be considered. It is known that water acts as a proton scavenger for the dimers, allowing polymerization to occur. However, an excess water vapor leads the formation of oxidizing agent crystallite domains which not effectively participate in the polymerization process. Therefore, Zuber et al. reported that the addition of the amphiphilic copolymer, PEG-ran-PPG, to the oxidizing agent solution suppresses crystallization due to steric hindrance and enhances the polymerization of defect-free PEDOT. Moreover, the copolymer allowed a wider humidity window to be used before the onset of crystal initiated defects is observed [52]. In a similar approach, and with the aim of suppressing the acidic side reaction and slowing down the oxidizing rate, the incorporation of pyridine as base inhibitor has been reported to yield films with conductivities up to $1000 \mathrm{~S} \mathrm{~cm}^{-1}[47,53]$.

In contrast to VPP, CVD involves a single-step polymerization where the vapors of both the oxidizing agent and the monomer meet and react at the target substrate (which is inversely placed above the oxidizing agent to minimize the probability of oxidizing particles falling on the substrate) for the oxidation of the EDOT (Figure 3A). To control 
the process, a high-vacuum atmosphere is required, and therefore the technique needs a vacuum chamber.

Compared with VPP, the options of oxidant are more limited in CVD, since many solid oxidizing agents have poor volatility, such as $\mathrm{Fe}(\mathrm{TOS})_{3}$. Therefore, metal halogen salts with high volatility are frequently employed, being $\mathrm{FeCl}_{3}$ the most used [54]. However, the use of other molecules such as $\mathrm{SbCl}_{5}$ and $\mathrm{VOCl}_{3}$ was recently reported, and films obtained employing $\mathrm{VOCl}_{3}$ showed better control of the flow rate in the CVD reactor compared to $\mathrm{FeCl}_{3}$, which yields a more controlled PEDOT growth $[55,56]$. Vapors of $\mathrm{MoCl}_{5}$ were also reported to produce high-quality PEDOT films [57]. Similar to what happens with the oxidizing agents, the employment of additives has rarely been reported for the CVD preparation of PEDOT films, due to the lack of suitable volatile additives.
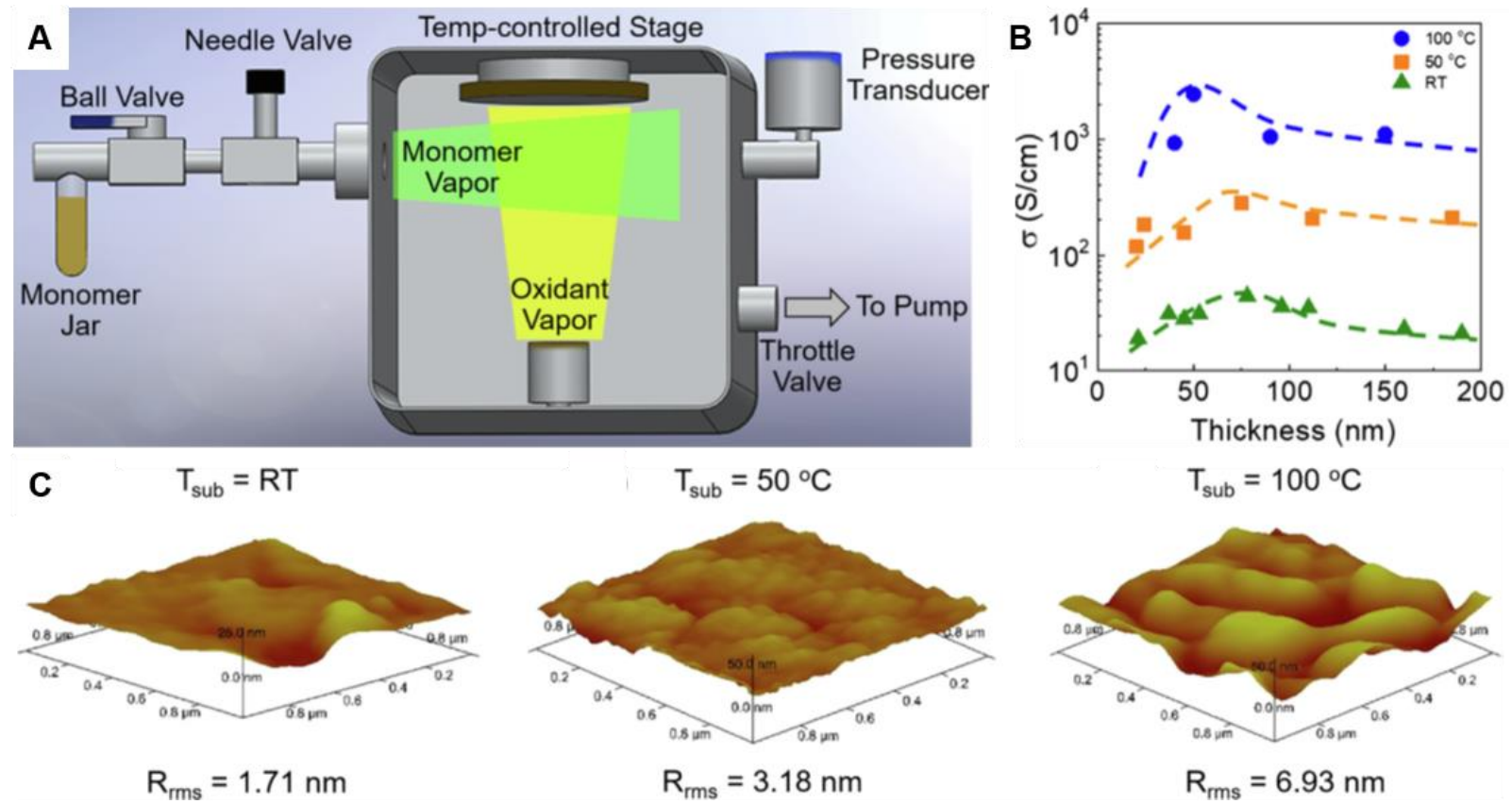

Figure 3. Scheme of a CVD reactor (A). Conductivity as a function of $T_{\text {SUB }}$ and film thickness (B). AFM topographic images of CVD PEDOT at different $\mathrm{T}_{\mathrm{SUB}}(\mathbf{C})$. Adapted with permission from reference [55]. Copyright 2020 Elsevier.

When considering the process variables affecting the final polymer film features, factors such as substrate temperature, pressure, vapor flow and location of the substrate can considerably modify the final features of the obtained films. In this sense, Drewelow et al. have recently studied the influence of the temperature of the substrate $\left(\mathrm{T}_{\mathrm{SUB}}\right)$ and the oxidizing agent ( $\mathrm{T}_{\mathrm{OXI}}$ ) on the growth kinetics of CVD PEDOT [55]. They found that at low $\mathrm{T}_{\text {SUB }}(\mathrm{RT})$, the growth rate follows an Arrhenius relationship with an activation energy of approximately $1.9 \mathrm{eV}$. However, at higher $\mathrm{T}_{\mathrm{SUB}}$, the growth rate becomes independent of $\mathrm{T}_{\mathrm{OXI}}$, and leads to enhanced crystalline structure in an edge-on packing orientation, as well as longer conjugation length. Therefore, these films show enhanced conductivity, carrier density and carrier mobility by the enhanced crystalline and chemical environmental structures in PEDOT grown at higher $\mathrm{T}_{\text {SUB }}$ (still in the modest temperature regime $\leq 100{ }^{\circ} \mathrm{C}$ ) (Figure 3B,C). The effect of temperature and the subsequent film structure on the electrochemical properties has been also recently studied by Moni et al. [58]. In another interesting study, Mirabedin et al. have reported detailed correlations between the deposition conditions and the film thickness, weight, chemical composition, morphology, and electrical conductivity for various $\mathrm{FeCl}_{3}$ /EDOT inlet ratios and substrate temperatures [59]. 


\subsection{PEDOT:PSS Films}

From the previous sections it can be noted that most of the examples of EDOT polymerization involve the employment of non-aqueous solutions, as the monomer shows poor solubility in water. A well-known method to overcome this problem involves the use of poly(styrenesulfonate) (PSS) as counterion. The story says that in an interesting example of (industrial) collaboration, scientists at Agfa were searching for new antistatic coatings when Jonas, a scientist at Bayer, suggested the use of PEDOT as antistatic material. Later, they attempted the oxidation of EDOT by persulfates in the presence of PSS in water, and obtained an aqueous dispersion of PEDOT stabilized with PSS as a counteranion, which showed great stability [29]. Precisely, the commercial availability and straightforward processing are the most important reasons why PEDOT:PSS dispersion is the most employed conducting polymer to date [60]. By making use of the aqueous microdispersion, conducting polymer films can be easily obtained on a variety of substrates through a wide range of deposition techniques such as spray deposition, screen-printing, dip-coating, spin-coating, drop-casting, and inkjet-printing, yielding surfaces with high electrical conductivity, optical transparency, and good stability in air, among other interesting features [16]. Moreover, by employing the commercial dispersion, even the 3D printing of PEDOT:PSS-based architectures has been recently reported [61,62].

On the other hand, the synthesis of PEDOT:PSS can also be performed at the laboratory scale. In this regard, the colloidal complex is often fabricated through solution-cast polymerization employing PSS as counteranion, as previously described. Briefly, the synthesis involves dissolving the polyanion in water, adding the EDOT monomer to produce an emulsion, incorporating the oxidant solution (a mixture of peroxodisulfates and Fe(III) salts), and, finally, purification of the dispersion (Figure 4A) [17]. The polyanion facilitates the solubilization of the EDOT monomer in water and the further dispersion and stabilization of the oligomers and polymers formed during the oxidation reaction. It should be noted that peroxodisulfates (e.g., $\mathrm{K}_{2} \mathrm{~S}_{2} \mathrm{O}_{8}, \mathrm{Na}_{2} \mathrm{~S}_{2} \mathrm{O}_{8}$ ) are usually employed as oxidizing agents in combination with small amounts of $\mathrm{Fe}(\mathrm{III})$ salts $\left(\mathrm{FeCl}_{3}, \mathrm{Fe}_{2}\left(\mathrm{SO}_{4}\right)_{2}\right)$ working as a catalyst, since multivalent cations (such as Fe(III)) could produce a complex with PSS, reducing the stability of the dispersion [63]. The procedure results in a very stable aqueous polyelectrolyte-conducting polymer microdispersion, in which soluble complexes are formed via electrostatic interaction between the positively charged PEDOT and PSS (Figure 4B).

A

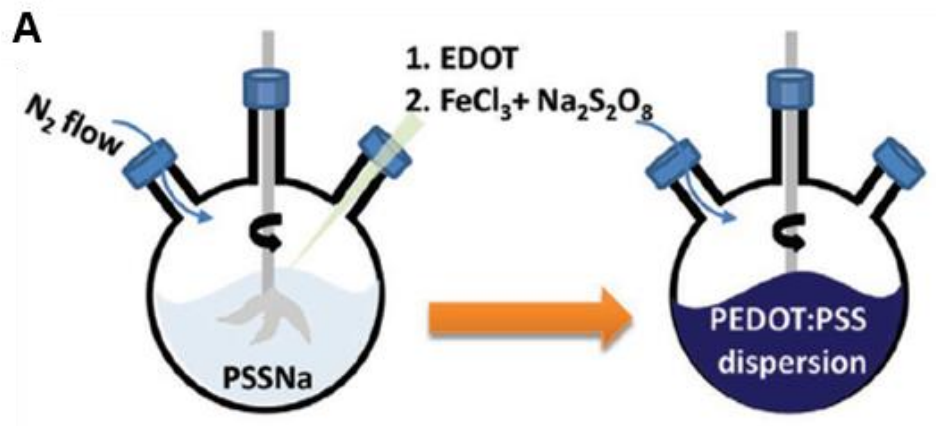

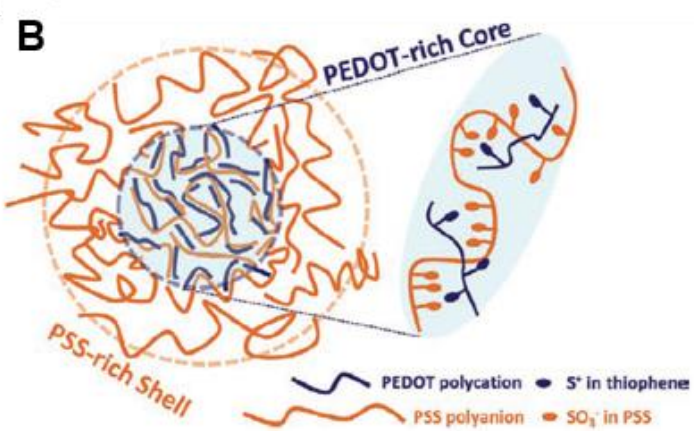

Figure 4. Scheme of PEDOT:PSS structure (A). Scheme of PEDOT:PSS polymerization (B). Adapted with permission from reference [17]. Copyright 2020 John Wiley and Sons.

As previously described for PEDOT:TOS, the processing conditions, such as the way of adding oxidizing agents, temperature, and mechanical stirring rate, among others, impact the physicochemical properties of the obtained PEDOT:PSS films. For instance, it has been reported that the one-time addition of the oxidizing agent (instead of step-by-step) and/or the increment in the acidity and temperature accelerate the polymerization rate, resulting in large size of PEDOT:PSS micelle, which subsequently leads to films with high roughness and poor electrochemical features [17]. 
It should be noted here that while the direct preparation of PEDOT:PSS films through the deposition of the commercial dispersion is very practical, the obtained films have usually low conductivities (between $10^{-4}$ to $1 \mathrm{~S} \mathrm{~cm}^{-1}$ ) compared with PEDOT obtained by other methods due to the overcompensating amount of insulating PSS in the films, which surrounds PEDOT and hampers electronic transfer [16,64]. Therefore, several strategies including irradiation by light, solvent, thermal and acid/basic treatments have been developed in order to enhance the features of the obtained films [65]. Finally, it is also relevant to note here that other polyanions have also been employed in order to develop novel water-soluble PEDOT complexes, such as pectin, hyaluronate, and dextran sulfate, as well as various polysulfonylimide-based polymers [66]. Although this subject will not be extensively covered in this review, interesting works on this matter have been recently published $[67,68]$.

\subsection{Summary}

In the previous sections several strategies have been described for the preparation of PEDOT and PEDOT:PSS films. Table 1 summarizes the most relevant advantages and issues of the PEDOT production by the methods discussed in this review.

Table 1. Main advantages and disadvantages of the PEDOT and PEDOT:PSS production methods.

\begin{tabular}{|c|c|c|}
\hline Approach & Advantages & Issues \\
\hline $\begin{array}{l}\text { Solution-cast } \\
\text { polymerization }\end{array}$ & $\begin{array}{c}\text { Simple procedure } \\
\text { Production of large-area films } \\
\text { Low cost }\end{array}$ & $\begin{array}{l}\text { Thickness may be difficult to control } \\
\text { Adhesion of the film can be a problem }\end{array}$ \\
\hline Electropolymerization & $\begin{array}{l}\text { Excellent connection between the substrate } \\
\text { and the film } \\
\text { Easy control of morphology and thickness } \\
\text { Simple copolymerization }\end{array}$ & $\begin{array}{l}\text { Conductive substrates are required } \\
\text { Potentiostat needed } \\
\text { Usually performed in organic solvents }\end{array}$ \\
\hline VPP and CVD & $\begin{array}{l}\text { Endows the modification of all-kind of } \\
\text { substrates } \\
\text { Production of very high-quality films }\end{array}$ & $\begin{array}{l}\text { More complex equipment required } \\
\text { Difficult to control the process conditions }\end{array}$ \\
\hline PEDOT:PSS dispersion & $\begin{array}{c}\text { Commercially available } \\
\text { Water dispersible } \\
\text { Wide variety of substrates can be modified } \\
\text { Different deposition techniques } \\
\text { Easy to scale-up }\end{array}$ & $\begin{array}{l}\text { Low conductivity } \\
\text { PSS hampers functionalization } \\
\text { Further treatment steps to } \\
\text { improve properties }\end{array}$ \\
\hline
\end{tabular}

\section{Functionalization Strategies for PEDOT and PEDOT:PSS Films}

In the field of organic bioelectronics, the coupling of the conducting polymer with the biological units represents the most relevant step in the fabrication of an efficient device [19]. In this sense, the different functionalization strategies of organic films pursue the same final objective, which is to bind a biomolecule securely and tightly. Moreover, the anchoring must not only by stable, but must also preserve the functionality of the bio-entity and the electronic properties of the undelaying conducting polymer. For instance, when considering antibody immobilization, a proper orientation of the biomolecule upon binding is required, as the binding sites should be accessible to the antigen [69]. In the same way, enzyme immobilization must preserve the native conformation of the protein and allow for reagent transport and successive product release [70,71].

In this regard, the biofunctionalization of conducting polymers can be addressed by two different ways, i.e., the bio-entity can be directly incorporated into or onto the organic film, or molecules or functional groups can be employed in order to generate a more biocompatible surface for, namely the incorporation of proteins or the improvement of cell adhesion [72]. When considering PEDOT, the one-step integration of bio-entities in the CP matrix represents a big challenge: as previously mentioned, the polymerization conditions 
are often aggressive since they involve the use of organic solvents and relatively high curing temperatures. These conditions affect the structural integrity of most biomolecules, thus, leading to diminished function. On the other hand, the lack of functional groups in pristine PEDOT films hampers the incorporation of biomolecules on its surface [20]. Therefore, the incorporation of biomolecules should ideally be performed after the preparation of the films, requiring the functionalization of the surface. In a similar fashion, when employing PEDOT:PSS films, the acidity/toxicity [22] and the overall anionic charge of the conducting polymer-polyelectrolyte complex restrict its interaction and integration with negatively charged entities, such as cells, DNA/RNA and most proteins at physiological $\mathrm{pH}$ conditions, requiring the deposited films to be further modified [68,73]. Hence, adapting and modulating the functionality of PEDOT and PEDOT:PSS films to further achieve effective biointerfaces for organic bioelectronics is needed.

In this section, we will focus on the incorporation of materials/functional groups to PEDOT and PEDOT:PSS films to further integrate or improve their interaction with bio-entities. Then, the different approaches for the functionalization of PEDOT will be divided in three main groups; (Section 3.1) the incorporation of moieties to an EDOT-like monomer and the further copolymerization with EDOT, (Section 3.2) the incorporation of the desired molecules to the polymerization solution and the subsequent polymerization; and (Section 3.3) the functionalization of PEDOT:PSS films. Predictably, the approach to be used will depend on the synthetic strategy employed. In this sense, most of the functionalization methods reported for electropolymerized PEDOT involve the synthetic modification of the EDOT monomer, while for PEDOT:PSS, the most employed strategy involves the mixing of the commercial solution with the additive/the modification of the film after preparation.

\subsection{Derivatization of the EDOT Monomer}

A well-known approach in order to obtain films which are prone to the incorporation of biological entities involves the polymerization of EDOT derivatives containing recognition elements/motifs or a functional group able to be modified in a subsequent step [74-76]. This strategy involves the use of organic chemistry strategies to obtain the desired EDOT derivative, and its subsequent polymerization by making use of the different oxidative methods. In this sense, when designing the monomer, one must consider that the polymer should retain its insoluble character, but also take into account that the incorporation of over-sized substituents can lead to an excessive solubility in the polymerization solvent [29]. Next, the obtained PEDOT-like polymer can either be a homopolymer, when only the derivatized monomer is used, or a copolymer, as EDOT can be employed to maintain the electrochemical properties of the films [20]. Therefore, the polymerization technique and the counteranion/dopant must be carefully selected to obtain the desired film properties.

In this regard, electropolymerization is the most employed technique to perform the oxidation of EDOT derivatives, since it allows for the simple control of the different electrochemical parameters during the polymerization procedure, facilitating the tuning of the monomer ratios in the obtained film. For instance, Bhagwat et al. have reported the synthesis of a carboxylic acid-functionalized-EDOT (EDOT-acid) monomer and the galvanostatic preparation of copolymer films with EDOT (PEDOT-acid) [77,78]. The PEDOT-acid copolymer films were electrochemically polymerized from a solution containing a monomer feed ratio of EDOT-acid and EDOT of 0.75 and 0.25 , respectively. Subsequently, the carboxylic moieties of the surface were employed to anchor the nonapeptide CDPGYIGSR (a peptide derived from the basement membrane protein laminin) as well as PEGylated CDPGYIGSR through the N-terminal of the peptide. The efficiency of the peptide together with the effect of the spacer molecule were evaluated for cell adhesion and differentiation in cell culture assays employing the rat pheochromocytoma (PC12) cell line. Peptide-modified films comprising the longest PEG spacer exhibited the best attachment and neurite outgrowth, providing an effective approach to biofunctionalize conducting polymer films [78]. 
Similarly, the incorporation of the acid moieties in the EDOT monomer was also found to enable the anchoring of the GGGGRGDS peptide, while maintaining the conductivity of the bioactive film. Subsequently, the PEDOT-acid-peptide films were able to increase the adhesion of primary rat motor neurons [79].

In another study, Ouyang et al. reported the synthesis of 1,3,5-tri [2-(3,4-ethylenedioxythienyl)]-benzene (EPh), a trifunctional crosslinking agent with three EDOTs around a central benzene ring, and its electro-copolymerization with EDOT [80]. Systematic variations in composition from mixed monomer solutions in acetonitrile were investigated, showing that the impedance increased with the EPh feed ratio. Moreover, the authors performed in vitro cell culture studies, showing that the PEDOT-co-EPh films with less than $1 \%$ of EPh exhibit excellent biocompatibility to PC-12 cell lines, with limited cytotoxicity and the ability to support neurite outgrowth.

Very recently, Mantione et al. developed different EDOT-based trimers beating ammonium and sulfonate moieties as novel water-soluble EDOT-based bioelectronic materials [81]. The trimers were able not only to electrochemically polymerize in acetonitrile, but also to chemically polymerize in water. Moreover, the trimers were effectively polymerized in vivo along the roots of living plants employing the activity of native peroxidase enzymes.

In an interesting approach, Wei et al. performed the synthesis and further polymerization of a dialkene functionalized ProDOT variant (ProDOT-diene). The ProDOT-diene monomer was either chemically or electrochemically polymerized, and then subsequently modified with alkyl, PEG, or ferrocene moieties via radical-based thiol-ene chemistry, indicating that the method allows for efficient, facile tuning of the surface chemistry of the films [82].

Hai et al. reported the synthesis of an EDOT derivative bearing an oxylamine moiety (EDOTOA), which was subsequently electrochemically polymerized with EDOT in aqueous solution employing $\mathrm{NaClO}_{4}$, yielding poly(EDOT-co-EDOTOA) films (Figure 5A). The feed ratio of EDOTOA in the electropolymerization solution was varied from 0 to $100 \%$, showing the best electrochemical properties for the films with 25\% EDOTOA, since the increase in the EDOTOA ratio hampered the charge transfer at the polymer/electrolyte interface. Then, these films were employed for covalently introducing sialyllactose on the films via glycosylation between the reducing end of lactose and the oxylamine unit in poly(EDOT-co-EDOTOA) (Figure 5B,C). Finally, the sialyllactose-grafted poly(EDOT-coEDOTOA) films were employed to perform the specific recognition of human influenza $A$ virus (H1N1) [83].

The solution-cast polymerization (and copolymerization) of EDOT derivatives containing recognition elements/motifs has also been reported to be an effective strategy to further incorporate biomolecules on films. For instance, Daprà et al. presented the preparation of PEDOT/PEDOT-OH platforms by the chemical copolymerization of both monomers and its further deposition on polymeric substrates [84]. Moreover, the incorporation of $\mathrm{OH}$ moieties enabled the further covalent attachment of two aptamer probes through EDC-NHS chemistry for the construction of biosensors. The constructed devices showed high sensitivity and selectivity for ampicillin and kanamycin A detection (depending on the aptamer employed) by electrochemical impedance spectroscopy (EIS). Finally, it was possible to detect ampicillin in a milk sample at a concentration below the allowed maximum residue limit (MRL).

In another interesting approach, Minudri et al. have very recently reported the synthesis of a cationic ammonium-modified EDOT monomer (EDOT-N), as well as its solution-cast polymerization as a homopolymer and also as a copolymer with EDOT, yielding stable films (Figure 6A,B) [22]. Moreover, the versatility of the PEDOT-N homopolymer was demonstrated by processing the polymer in two different ways. First, PEDOT-N was assembled from water dispersions as thin films obtained by layer-by-layer (LbL) assembly employing PEDOT:PSS as cationic counterpart. In addition, the straightforward preparation of conducting PEDOT-N hydrogels was studied, employing PEGDA-diacrylate and a 
photoinitiator (Figure 6C). The authors also showed that the PEDOT-N films showed good biocompatibility in the presence of the human embryonic kidney-293 cell line, displaying great potential in bioelectronics applications (Figure 6D).

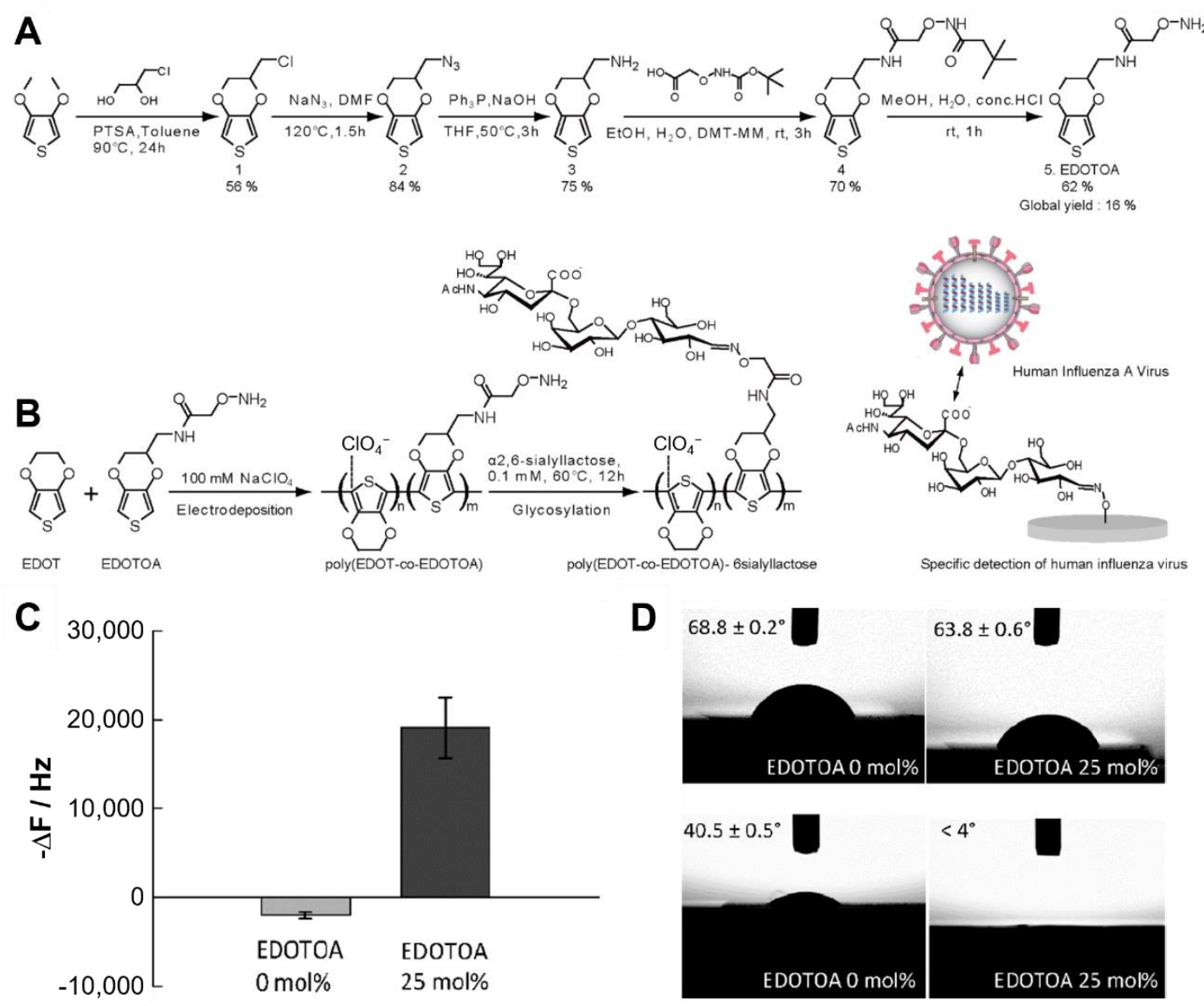

Figure 5. Synthetic route for the preparation of sialyllactose-grafted monomer (A). Scheme of the copolymer synthesis and glycosylation (B). Changes in the surface mass (C) and wettability (D) of the functionalized polymer upon glycosylation. Adapted with permission from reference [83]. Copyright 2017 American Chemical Society.

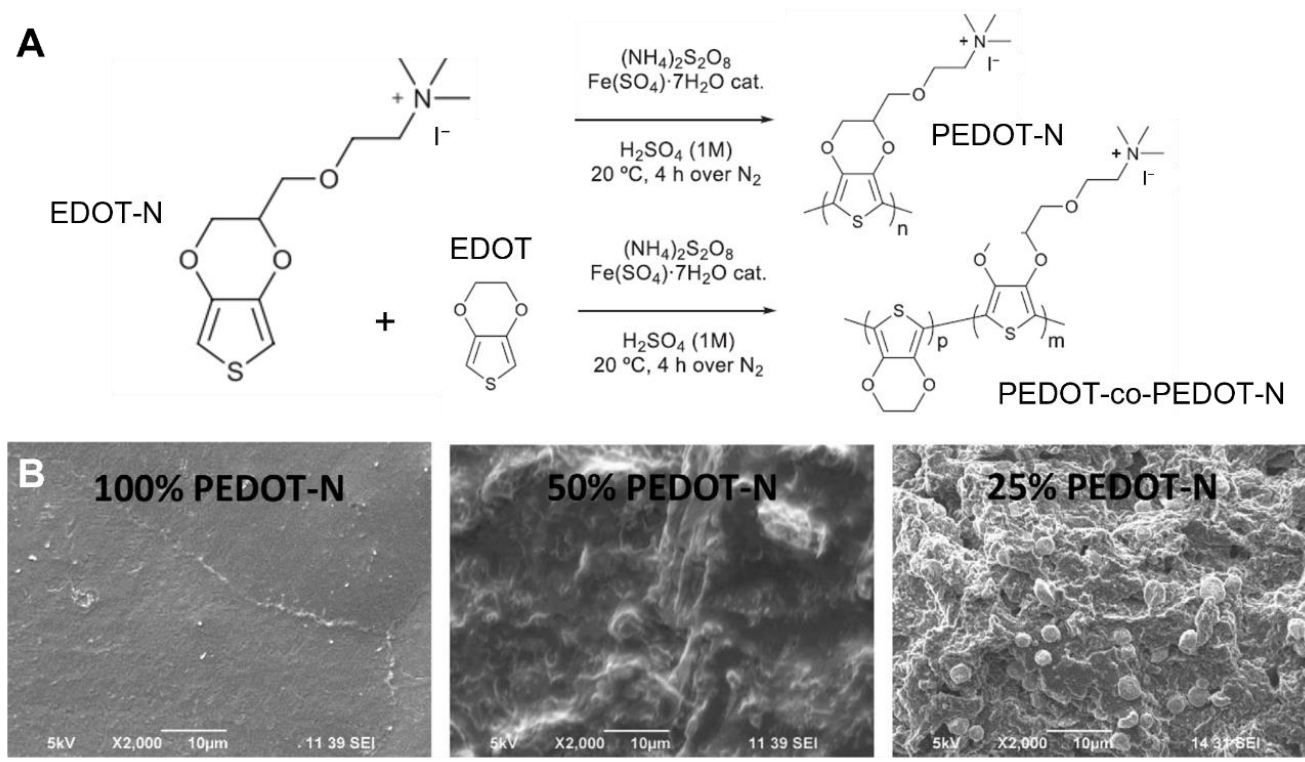

Figure 6. Cont. 
C

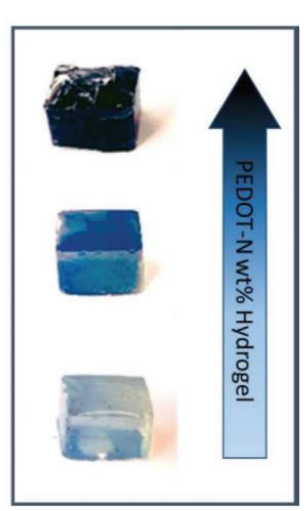

D

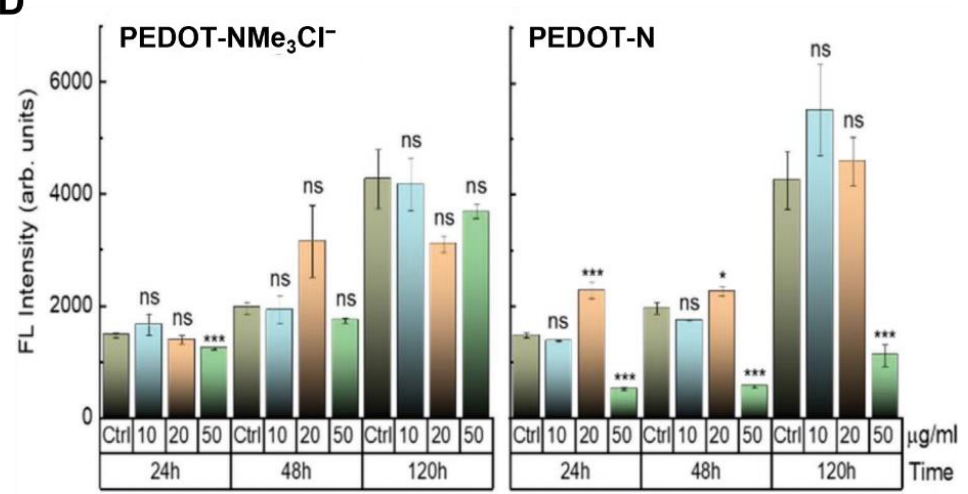

Figure 6. Scheme of the synthesis of PEDOT-N homopolymer and PEDOT-co-PEDOT-N copolymers (A). SEM of thin films obtained by casting aqueous solutions of PEDOT-N homopolymer, $50 \%$ PEDOT$\mathrm{N}$, and 100\% PEDOT-N (B). Pictures of hydrogels containing a decreasing amount of PEDOT-N polymer (C). Cell proliferation tests on HEK-293 cell models exposed to different cationic PEDOT-N polymer dispersion concentrations, at different time points after plating. Two counter ions were studied: $\mathrm{Cl}-$ and I- (left and right panel, respectively). Error bars represent the standard deviation of the mean. (D) Adapted with permission from reference [22]. Copyright 2020 John Wiley and Sons.

\subsection{Addition of Molecules to the Polymerization Solution}

Another effective way to tailor the biocompatibility properties of PEDOT films involves the addition of molecules bearing the desired moieties/motifs to the polymerization solution. Briefly, the technique involves the addition of the entity to the oxidizing agent solution (or electrolyte in the case of electropolymerized PEDOT) and the subsequent oxidation of the monomer, either performed by solution-cast polymerization, electropolymerization or VPP/CVD. Concerning the additives, a frequent strategy to increase the biocompatibility of the PEDOT-based surface is the inclusion of hydrophilic polymers, such as synthetic polyelectrolytes (polycations or polyanions), natural polypeptides and even neutral polyethylene glycol (PEG) moieties.

In this regard, while the preparation of PEDOT-like films employing derivatized EDOT monomers have not been widely reported for VPP, the functionalization of PEDOT by adding molecules/moieties to the oxidant solution and the further polymerization of the monomer by VPP has been extensively explored, yielding composite films where the additive was intimately connected to the PEDOT matrix. In this way, the incorporation of polyethylene glycol (PEG)-like molecules has been reported as an efficient approach towards the generation of biocompatible PEDOT surfaces. For instance, PEG and a carboxylic acid-functionalized PEG (PEG(COOH)) were incorporated in PEDOT:TOS oxidative solution and films were obtained through VPP. Afterwards, EDC-NHS chemistry was employed in order to incorporate fluorescent proteins onto the functionalized PEDOT films via the carboxyl moieties of PEG(COOH) [85]. Moreover, the addition of PEG has been also reported to improve the conductivity of PEDOT composite films obtained through VPP, enabling the successful fabrication of organic electrochemical transistors by making use of the composite film as channel material [85,86].

In another interesting approach, a conducting composite polymer film was obtained by VPP of EDOT in the presence of a biocompatible polyanion derived from the partial sulfonation (32\%) of statistical ethylene vinyl alcohol copolymer (EVAL32) [87]. A methanol/water solution containing EVALS32 and the oxidant FeTOS 3 was spin-coated on glass slides, which were subsequently exposed to EDOT vapors, yielding EVALS32PEDOT composite films with good conductivity, transparency, and stability in water. The addition, the partially sulfonated EVAL matrix yielded conducting platforms possessing residual hydroxyl groups able to be submitted to further chemical modification, including crosslinking, and grafting with bioactive molecules. 
Similarly, Bongo and coworkers reported the incorporation of gelatin, a derivative of the extracellular matrix protein collagen, into PEDOT:TOS films via VPP. The integration of gelatin not only retained the electrochemical properties of the conducting polymer film, but it also specifically supported bovine brain capillary endothelial cell adhesion and growth. This results indicated that the functionality of the biomolecule was maintained and demonstrated the future potential of this type of conducting biocomposites in order to promote cell adhesion in electrically active materials for tissue engineering [88].

The addition of molecules to the polymerization solution of solution-cast PEDOT has also been reported as an effective strategy to modulate the biofunctional properties of the conducting films. In this sense, the incorporation of polyamines to the polymerization solution has gained interest in the recent years. For instance, the successful preparation of PEDOT-polyallylamine hydrochloride (PEDOT-PAH) films on different substrates such as Plexiglas [89], PET [90], and interdigitated Au micro electrodes [91] has been reported recently, enabling the fabrication of different bioelectrochemical devices upon further functionalization of the composites films (Figure 7A,B). The effect of the addition of different amounts of polyamine to the polymerization solution was studied, showing that the variation of the PAH:PEDOT ratio in the polymerization solution allowed for the tuning of the electronic and ionic features of the films [91]. Moreover, carbohydrate motifs were covalently anchored to the primary amine groups using divinylsulfone chemistry (Figure 7C), and the recognition-driven assembly of the lectin Concanavalin A (ConA) and the enzyme glucose oxidase (GOx) on the mannosylated surfaces was performed (Figure 7D). Finally, the detection of glucose by the functionalized electrodes was studied [89].

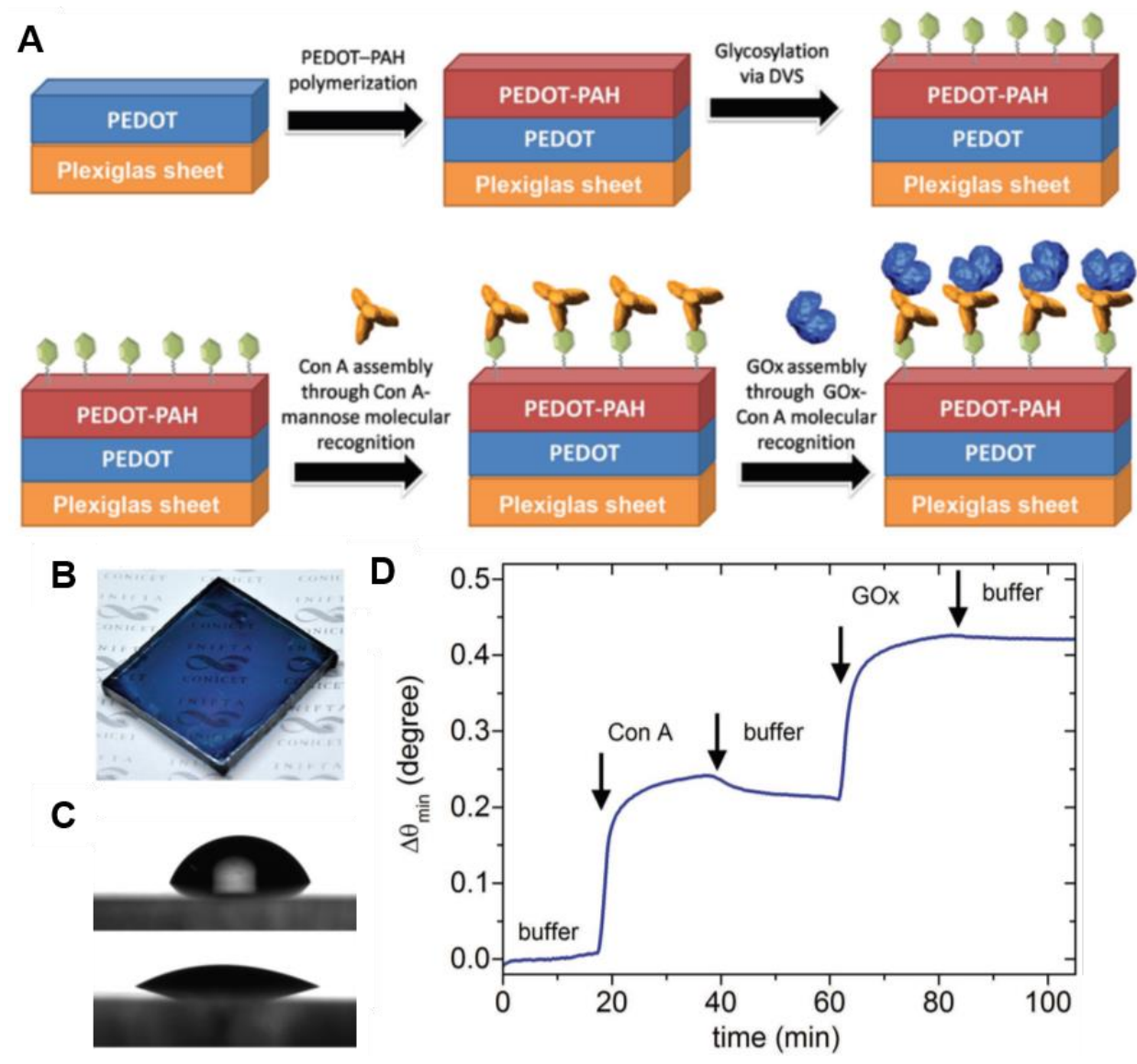

Figure 7. Scheme of the PEDOT-PAH composites preparation and biofunctionalization through mannosylation, and ConA and GOx recognition (A). Photograph of a Plexiglas substrate modified with the composite PEDOT-PAH (B). Contact angle images of PEDOT-PAH before (top) and after (down) mannosylation (C). SPR sensogram for the assembly of Con A and GOx on a mannosylated sensor (D). Adapted with permission from reference [89]. Copyright 2017 John Wiley and Sons. 
The incorporation of biopolymers has also been reported as a useful strategy for the improvement of the biofunctionalization properties of PEDOT-like films. In this sense, Zamora et al. showed the fabrication of nanoporous PEDOT-based materials through an assisted processing approach using starch/ $\mathrm{K}$-carrageenan aerogels as templates. The polysaccharide aerogel morphology was employed to yield meso- and macroporosity to the conducting polymer, while the carrageenan was maintained in the polymer as a dopant, improving the electrical and mechanical properties of the resulting nanostructured material. Moreover, the resulting composite materials were compatible with the viability of SH-SY5Y human neuroblastoma cells differentiated to a neuronal phenotype [92].

Moreover, Harman et al. reported the preparation of films composed of a complex between PEDOT and the sulfonated polysaccharide polyanion dextran sulfate (DS). The films were obtained via oxidative chemical polymerization of EDOT in the presence of the DS polyanion, a surfactant and an anticoagulant and subsequent drop-casting, showing conductivity similar to that obtained for PEDOT:PSS films. Moreover, water dispersions of the polymer were successfully processed by different methods as drop-casting, spraycoating, inkjet-printing and extrusion-printing. Finally, the addition of the PEDOT:DS dispersion to the growth medium of L-929 cells did not affect the development of the cells, in opposition to PEDOT:PSS, indicating that the PEDOT:DS dispersions show greater potential for biological applications [93].

On the other hand, the incorporation of bio-functionalizable molecules in the electrolyte solution and the further electropolymerization of EDOT has also been described by different authors. In this sense, Xia et al. reported an interesting approach where collagen, a cell-adhesion protein, was added to the electropolymerization solution, and the deposition of the film onto Au substrates was subsequently performed via galvanostatic polymerization. When the polymer was deposited at lower current densities, the obtained composite film showed good electroactivity, and SEM images revealed that the PEDOT/collagen film exhibited a network-like structure. Moreover, optical microscopy examination showed that rat pheochromocytoma (PC12) cells were preferentially seeded on PEDOT/collagen other than PEDOT $/ \mathrm{LiClO}_{4}$, indicating that collagen is highly bioactive for cell adhesion [94].

In another interesting example, Vara et al. showed the electrochemical deposition of PEDOT doped with poly[(4-styrenesulfonic acid)-co-(maleic acid)] (PEDOT:PSS-coMA) on gold electrodes and carbon microfibers (MFs) employing constant anodic current. The authors showed that the obtained film was able to be further modified with linked $\mathrm{N}$-Cadherin (NCad), a calcium-dependent adhesion molecule that has a major role in axonal guidance and fasciculation in vivo and is also required for neuronal migration and synaptic plasticity. In the first place, the procedure for the protein attachment involved the binding of a goat antibody raised against human IgG (Fc specific) through EDC-NHS chemistry, making use of the carboxylic moieties in PSS-co-MA and the amino residues of the protein. Then, a recombinant human NCad-Fc chimera that combines human NCad with the Fc region of human immunoglobulin G1 (IgG1) was linked to the surface. The applicability of the electrodes for neural activity recording was assessed in the acute in vitro rat hippocampal slice, showing that $\mathrm{N}$-Cadherin biofunctionalized electrodes display advantageous properties for the selective detection of the activity of neighboring neurons. Furthermore, measurements of evoked activity indicated that synaptic physiology was unaffected by the electrodes [95].

Promsuwan et al. showed a straightforward approach to incorporate and regulate the proportion of carboxylate functional groups on PEDOT films [4]. The incorporation of the carboxylate moieties was performed through electropolymerization of EDOT with $\mathrm{LiClO}_{4}$ and the presence of different carboxylates, i.e., acetate $\left(\right.$ mono- $\left.\mathrm{COO}^{-}\right)$, malate $\left(\right.$di- $\left.\mathrm{COO}^{-}\right)$, citrate (tri- $\mathrm{COO}^{-}$), and poly(acrylamide-co-acrylate) (poly-COO${ }^{-}$). The authors showed that the polymerization efficiency, surface carboxylate density, electrochemical kinetics, and cycling stability of the resulting PEDOT:COO ${ }^{-}$interfaces increased with the molecular weight and carboxylate groups of the organic acid additives, possibly due to the increased doping efficiency and minimized leakage of the high-molecular-weight poly(acrylamide- 
co-acrylate) in the PEDOT:poly-COO ${ }^{-}$matrix (Figure 8A-H). Moreover, the carboxylate groups of PEDOT:poly-COO- served as anchoring sites for stable covalent coupling of enzyme lactate dehydrogenase (LDH) via EDC-NHS chemistry, enabling the fabrication of biosensors for lactate detection with high sensitivity, good selectivity, and reproducibility (Figure 8I).
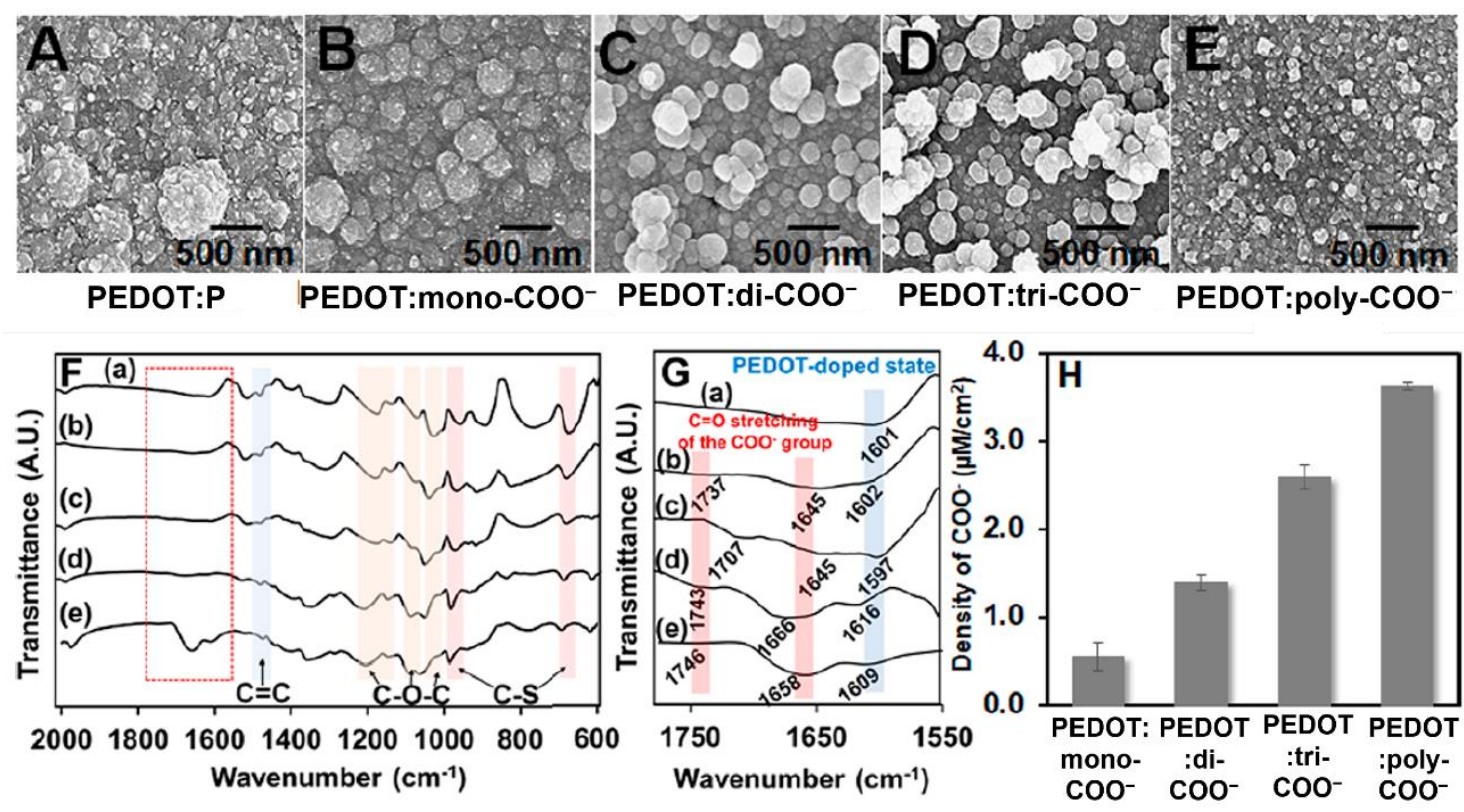

I

- Carboxylate-rich PEDOT-COOinterface for bio-conjugation - Improved stability

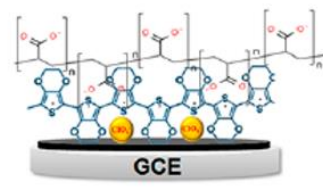

PEDOT-poly-COO-interface
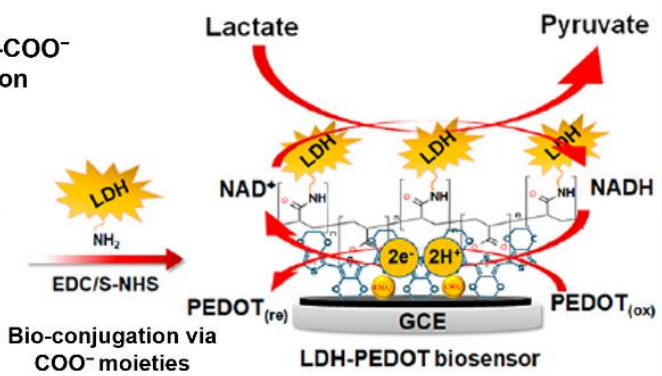

Figure 8. SEM images of different PEDOT:COO- films (PEDOT:P is for PEDOT without COO-). The scale bar is $500 \mathrm{~nm}$ (A-E). FTIR spectra in the range $2000-600 \mathrm{~cm}^{-1}$ (F) and 1750-1550 $\mathrm{cm}^{-1}$ (G) for (a) PEDOT:P, (b) PEDOT:mono-COO-, (c) PEDOT:di-COO-, (d) PEDOT:tri-COO-, and (e) PEDOT:poly-COO-. Density of carboxylate groups of different PEDOT:COO - films measured by the TBO assay $(\mathbf{H})$. LDH anchoring and lactate biosensing mechanism at the LDH-PEDOT interface (I). Adapted with permission from reference [4]. Copyright 2020 American Chemical Society.

\subsection{Functionalization of PEDOT:PSS Films}

As the commercial PEDOT:PSS formulation is obtained ready to deposit, there are two main ways to functionalize the films: (Section 3.3.1) by blending the additive with the commercial PEDOT:PSS solution; or (Section 3.3.2) by performing the functionalization after the deposition of the film.

\subsubsection{Blending Methods}

Considering the blending of certain molecules to improve the bio-functionalizable features of PEDOT:PSS films, Polino et al. showed the fabrication of conducting films based on different PEDOT:PSS and polyethylene glycol diacrylate (PEGDA) blends. The authors studied the influence of PEGDA adding from 2 to $17 \mathrm{wt} . \%$ to the PEDOT:PSS commercial solution on the deposition of films via spin- and spray-coating and evaluated their optical, electrochemical, and morphological features. They found that the film fabricated with $8 \%$ of PEGDA yields the best compromise between electrical and optical properties. Moreover, 
the electrodes were tested as culture support for primary fibroblasts, and showed high biocompatibility and cell spreading in all the cases [96].

In another interesting example, Abedi et al. showed the fabrication and characterization of chitosan (CS) scaffolds containing PEDOT:PSS. Chitosan scaffolds containing $0.3,0.6$ and $1 \mathrm{wt} . \%$ of PEDOT:PSS were fabricated through electrospinning. The electrical and mechanical properties, as well as biocompatibility and cell viability of scaffolds were investigated, with the result that addition of PEDOT:PSS to chitosan scaffold not only enhances the mechanical properties and electrical conductivity of electrospun scaffolds, but also improves their biocompatibility and cell viability. These authors showed that the results obtained from scaffolds compared with the properties of native myocardium extracellular matrix reveal its potential application for cardiac tissue engineering [97].

Kumar and collaborators reported the deposition of PEDOT:PSS/polyvinyl alcohol (PVA) nanofibers on conducting paper (Figure 9A). PVA powder was added to PEDOT:PSS, stirred and heated to ensure a homogenous solution which was subsequently electrospun, yielding PEDOT:PSS/PVA nanofibers (EsNf) (Figure 9B,D). The modified paper electrodes were further biofunctionalized with the monoclonal carcinoembryonic antibodies (antiCEA) through physical adsorption, as a strong interaction occurred between negatively charged PEDOT:PSS/PVA composite and positively charged end $\left(\mathrm{NH}_{3}{ }^{+}\right)$of the anti-CEA molecules (Figure 9C). Later, BSA was added to block unspecific sites of to the anti-CEA functionalized PEDOT:PSS/PVA electrode (Figure 9E). Finally, the platform was shown to be useful toward the electrochemical detection of cancer biomarker (CEA) [98].

A

A
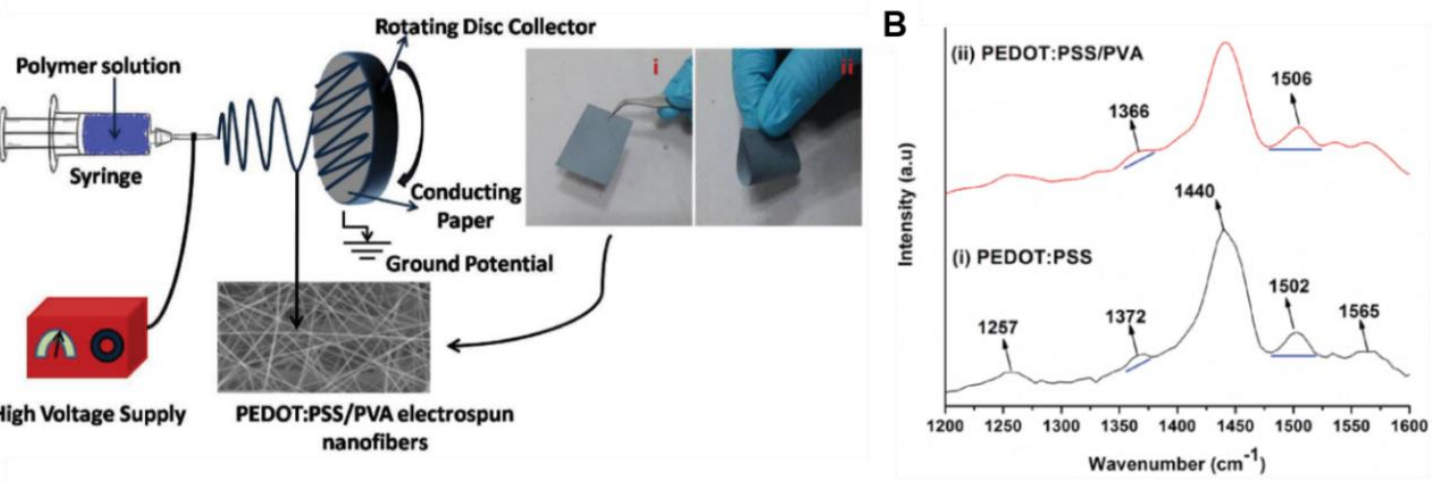

C
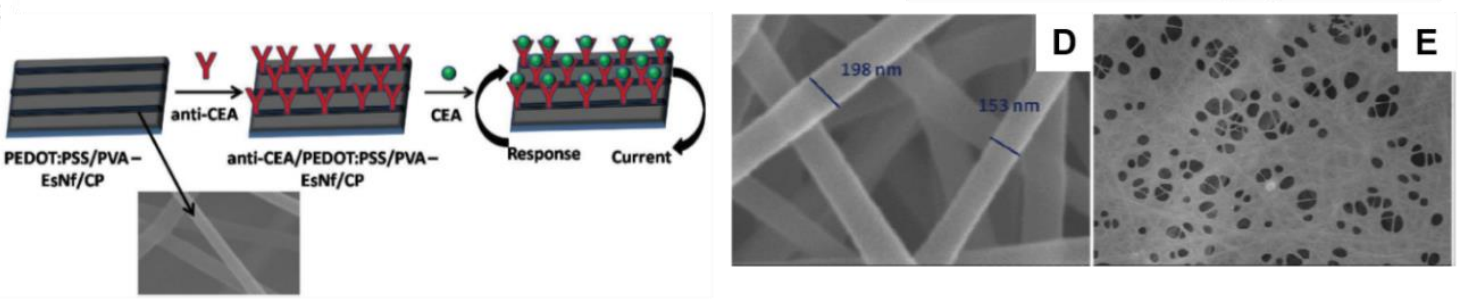

Figure 9. Scheme of the electrospinning setup for the obtention of PEDOT:PSS/PVA nanofibers (A). Raman spectra of PEDOT:PSS and PEDOT:PSS/PVA (B). Scheme for the biofunctionalization of the PEDOT:PSS/PVA nanofibers for carcinoembryonic antigen (CEA) detection (C). SEM images of PEDOT:PSS/PVA-EsNf/CP (D) and anti-CEA/P EDOT:PSS/PVAEsNf/CP (E). Adapted with permission from reference [98]. Copyright 2016 John Wiley and Sons.

Employing a similar approach, Strakosas et al. showed the addition of PVA to PEDOT:PSS before film casting, and the further chemical vapor deposition of a silane reagent (3-glycidoxypropyltrimethoxysilane, GOPS) onto the film. The modification of the surface with the silane reagent allowed for the further incorporation of polypeptides/proteins under basic conditions through the formation of a secondary amine bond with the biomolecule. Later, fluorescein isothiocyanate labelled poly-L-lysine (FITC-PLL) was immobilized on the surface, allowing for fluorescence investigations of the biofunctionalized pattern. A second relevant test of the effectiveness of this biofunctionalization method involved the immobilization of the enzyme glucose oxidase (GOx) on PEDOT:PSS:PVA films, yielding 
to the successful development of a glucose sensor. Moreover, the interaction of the PLL patterned surfaces with PC12 cells was studied, showing an excellent confinement of the cells to the patterned PEDOT:PSS:PVA. A live/dead cell staining assay showed that the cells were alive after 5 days, indicating that the PEDOT:PSS:PVA material is biocompatible for this application and period [99].

\subsubsection{Post-Functionalization of PEDOT:PSS Films}

The post-functionalization of the deposited PEDOT:PSS films has been also reported as a strategy to enhance the biocompatibility features of the surface. In this regard, He et al. reported the spin-coating deposition of PEDOT:PSS films and their further modification with a silane in order to improve the bacteria capturing properties of the surface [100]. First, the films were spin-coated and treated with oxygen plasma for different times. By this way, different hydroxide radical densities were generated, which were further employed to immobilize the anti-E. coli O157:H7 antibody. Next, the functionalized films were employed as channel material of OECTs to perform the detection of E. coli O157:H7 captured on the surface.

In another interesting approach, Ferlauto et al. reported the deposition of PEDOT:PSS and the further electrodeposition of an alginate layer (Figure 10A). The authors studied the mechanical properties of the hydrogel employing imaging techniques (Figure 10B-C), while the electrode arrays were electrochemically characterized at each fabrication step, and successfully validated both in vitro and in vivo (Figure 10D-F). It was shown that the use of alginate as a soft coating of microelectrode arrays is an attractive strategy to both reduce the mechanical mismatch at the electrode-tissue interface and improve the electrochemical properties of microelectrodes, leading to a lower electrical noise during neural recordings [101].

Collazos-Castro et al. have reported the deposition of PEDOT:PSS films and their post-functionalization with the polycationic polylysine (PLL) [102]. First, PEDOT:PSS was deposited on Au-coated glass slides and the electroadsorption of PLL was subsequently performed. The incorporation of the polycation enabled long-term neuronal survival and growth on the functionalized films. The authors also showed that the incorporation of an additional layer of PSS or heparin on the films resulted in a strong inhibition of neurite extension. Moreover, the binding of basic fibroblast growth factor (bFGF) to heparin inhibited neurons but enhanced precursor cells proliferation and migration.

Another interesting approach for the post-functionalization of PEDOT:PSS films towards organic bioelectronics applications involves the use of the Layer-by-Layer technique. The layer-by-layer assembly is a well-known strategy to simply functionalize charged surfaces by alternatively adsorbing negatively and positively charged entities $[11,103,104]$. The approach was first reported for the deposition of polyelectrolytes from water solutions, but presently it has been expanded to all kinds of solvents, molecules, and deposition strategies [105]. Therefore, the method allows the simple functionalization of the surface with a wide range of elements that include proteins, enzymes, polyelectrolytes, and phospholipids, among others [106,107]. Interestingly, this technique has been employed for the functionalization of PEDOT:PSS films since the polyanion in the conducting polymer matrix can act as an "initiator layer" for the beginning of the Layer-by-Layer architecture construction. Therefore, several authors have reported the functionalization of PEDOT:PSS films by this technique.

For instance, Pappa et al. have recently reported the functionalization of PEDOT:PSS films by the Layer-by-Layer approach in order to fabricate organic transistors devices which are able to immobilize and monitor nucleic acids [108]. First, the authors deposited a PEDOT:PSS film on transistors channels, and the sulfonate moieties of the polyanion were employed to initiate the deposition of PLL and PSS layers. The authors studied the multilayer assembly process on the films as well as its the effect on the transistor features. Moreover, they made use of the functionalized platform to control and monitor 
the electrostatic adsorption of a single-stranded negatively charged mRNA (messenger ribonucleic acid) onto the positively charged PLL layer of the LbL assembly.

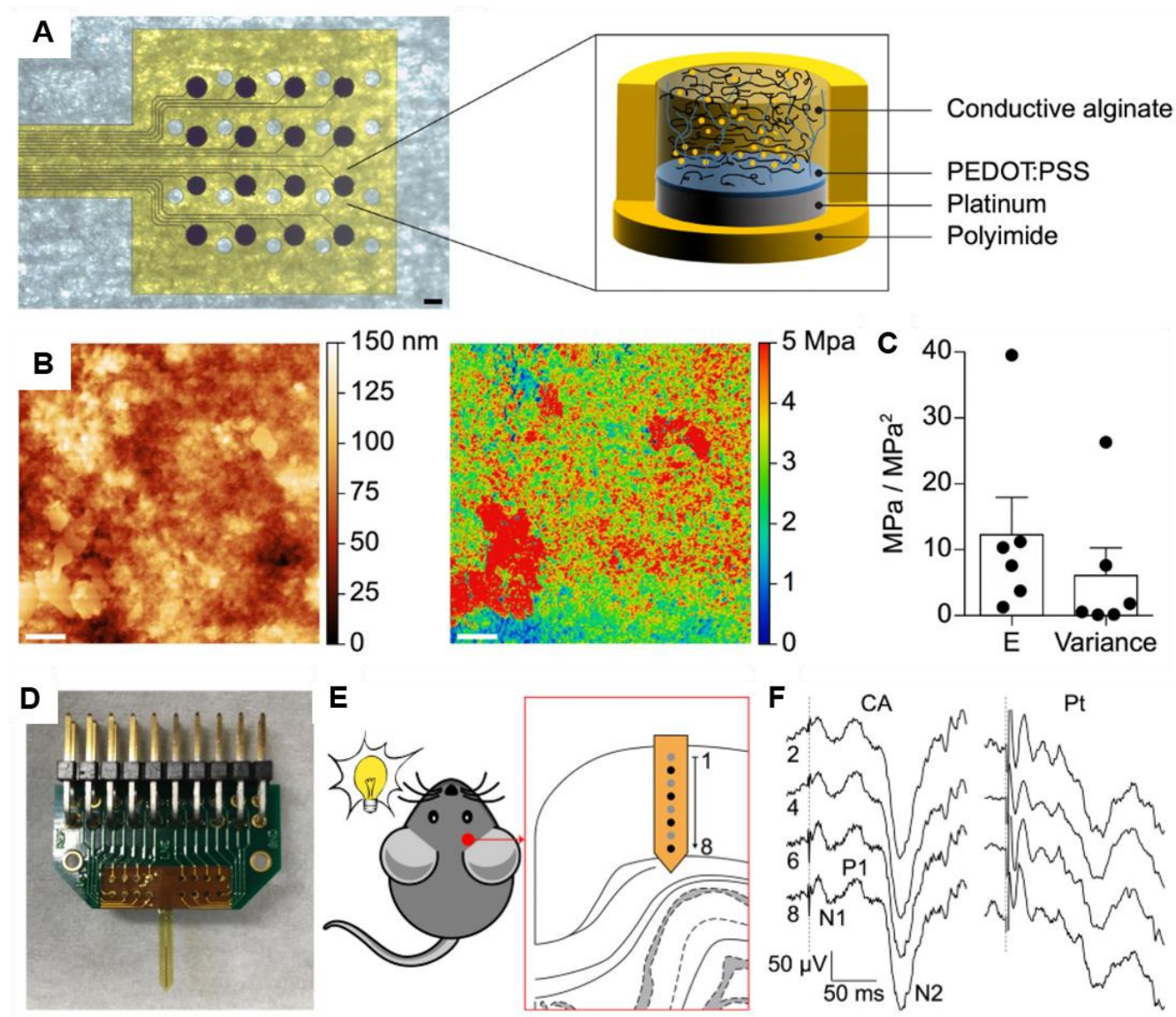

Figure 10. Pictures of the g-MEA $400 \mu \mathrm{m}$-diameter electrodes (the scale bar is $400 \mu \mathrm{m}$ ) and scheme of the soft CA-based electrode structure (A). AFM topography map of a $3 \mu \mathrm{m} \times 3 \mu \mathrm{m}$ surface area of the alginate modified electrodes (left) and the corresponding elastic modulus surface map (right). The scale bar is $400 \mathrm{~nm}$ (B). Quantification of the mean ( \pm SEM) stiffness and variance $(n=6$ electrodes, 1 map per electrode) (C). Validation in vivo of the modified electrodes (D-F). Picture of a penetrating MEA with CA electrodes (D). Experiment sketch (E). Representative single-sweep recordings from 4 (out of 8) PEDOT-alginate-based electrodes (F). Adapted under the terms of the CC-BY-4.0 license [101]. Copyright 2018, The author(s).

In another interesting report, Hardy et al. demonstrated the fabrication and evaluation of conducting biomaterials that encourage cell alignment made of PEDOT:PSS-chitosan LbL films [109]. First, a PEDOT:PSS layer was deposited on APTES-functionalized glass substrates, and next chitosan and PEDOT:PSS were alternatively deposited by means of an automatic system. The films were terminated with a layer of gelatin to improve cell adhesion, and the features of the films were studied. Finally, fibroblasts were cultured on them, showing adhesion and proliferation in vitro, and responding to a DC current passed through the films by aligning with the current.

By making use of the same approach, David et al. reported the incorporation of glucose oxidase on PEDOT:PSS films in order to fabricate a glucose sensor [110]. First, PEDOT:PSS was deposited on Au substrates, and the LbL assembly was performed employing a chitosan-modified graphene and the enzyme glucose oxidase. The films were characterized, and the analytical parameters of the biosensors were evaluated, showing the successful fabrication of a platform able to detect glucose down to $41 \mu \mathrm{M}$. Similarly, Daikuzono et al. showed the fabrication of a microfluidic electronic tongue by the assembly of PEDOT:PSSPAH Layer-by-Layer films on gold interdigitated electrodes [111]. 


\subsection{Summary}

From the studies presented in this section some generalities about the different functionalization methods can be obtained. In this regard, when considering the derivatization of the EDOT monomer, the procedure enables the rational design of the conducting polymer surface, allowing the generation of films with very specific properties. However, this approach needs the employment of organic chemistry strategies to successfully obtain the derivatized monomer. Moreover, the alteration of the structure of the monomer can yield undesirable results such as changes in solubility and different polymerization rate, which subsequently lead to changes in the required polymerization conditions. Furthermore, the effect on the characteristics of the obtained films, such as changes in morphology and electrochemical features, must also be considered.

On the other hand, the incorporation of additives such as small molecules, polymers and proteins to the polymerization solution involves a very simple way to modify the properties of the PEDOT polymer without the requirement of complex synthetic procedures. Moreover, the polymerization conditions are usually not affected by the addition of the desired molecule, and the control of the density of moieties can be easily performed by tuning the additive-monomer ratio. However, this approach can also negatively affect the electrochemical properties of the conducting composite, as the incorporation of an insulating element (as most of the additives) can hamper the different charge transfer processes throughout the composite film.

Regarding the functionalization of PEDOT:PSS films, the two different approaches discussed here are easy to perform and control. On the other hand, as PEDOT:PSS already contains a relatively high amount of a non-conducting component, the adding of another isolating molecule in or on top of the film can yield surfaces with poor conductivity. Moreover, both strategies are inherently conditioned by the features of the polyelectrolyte-conducting polymer complex (i.e., the presence of the polyanion), and therefore the options for the incorporation of molecules are limited. Finally, when considering the post-modification of deposited PEDOT:PSS films, the process will be performed mainly on the surface of the film, which could hamper ion diffusion when considering bioelectronics applications.

\section{Bioelectronics Applications Based on PEDOT and PEDOT:PSS Films}

So far, we have discussed the preparation methods of PEDOT-based films as well as several approaches for their functionalization towards the generation of biocompatible surfaces. In this section, we will highlight applications of PEDOT-based devices that benefit from this improved biocompatibility. The section will involve the description of bioelectronic devices involving the straight electrochemical connection of the conducting film with the bio-entity, such as organic electrochemical transistors, biosensors, and electrophysiological recording devices as well as scaffolds for cell interfacing and tissue engineering. In this regard, OECTs will be described separately due to their increasingly growing relevance and multiple applications within the organic bioelectronics field.

\subsection{Organic Electrochemical Transistors}

Briefly, an organic electrochemical transistor (OECT) comprises a three-terminal device immersed in an electrolyte solution, where an organic semiconductor film forms the channel of the device between the source (S) and the drain (D) electrodes. The gate electrode (G) modulates the conductivity of the channel and, therefore, the current that flows between the $S$ and D electrodes ( $I_{D S}$ ). Consequently, OECTs can operate as a switch, where $V_{G}$ is the input and $\mathrm{I}_{\mathrm{DS}}$ is the output. Moreover, they can also function as amplifiers, where the power of the input signal is amplified on the way to the output [6]. In contrast to traditional field-effect transistors, OECTs employ ion injection from the electrolyte solution to modulate the conductivity of the organic channel, resulting in devices with record-high transconductance [91,112]. This feature, together with the soft nature of the organic channel, makes OECTs particularly efficient for the development of organic bioelectronic devices. 
Moreover, the possibility of the channel to have a direct contact with the electrolyte/tissue and the use of low gate and drain voltages are also interesting features for this area of application.

PEDOT:PSS is the most used material for the fabrication of OECTs, and several bioelectronic devices for health care-related applications and biomedical research have been developed employing this channel material $[8,113]$. For instance, Hempel et al. have recently studied the adhesion and interaction of cells with PEDOT:PSS-based OECTs down to a single cell resolution using an impedimetric readout method [114]. Rivnay et al. have also demonstrated the high-quality recordings of human brain rhythms with PEDOT:PSS OECTs [115]. Furthermore, Liang et al. have reported that flexible PEDOT:PSS-based OECTs are able to record cardiac action potentials, competing with hard-material-based OECTs [116], among other numerous examples.

However, one of the main problems of PEDOT:PSS OECTs involves their operation in depletion mode, meaning that the organic channel is originally in its conducting state, and requires the application of a gate voltage to switch off the device [117]. This feature leads to undesirable consequences when operating in solution, since relatively high $\mathrm{V}_{\mathrm{GS}}$ to maintain the device in the "OFF" state are required and high operating currents are produced, prompting parasitic reactions with dissolved oxygen and water, and inducing the deterioration of the device $[13,91]$.

These factors have motivated the development and use of novel PEDOT-based channel materials for OECTs. For instance, Cea et al. have recently developed enhancement-mode ion-gated organic electrochemical transistors (E-IGTs) employing a blend of PEDOT:PSS and PEI as channel material and chitosan as ion membrane [118]. The devices showed high transconductance and rapid transient response with a stable operation over an extended period of time and allowed the fabrication of integrated circuits. Moreover, the E-IGTs were employed in a wide range of neurophysiological applications, including electromyography, electrocardiography, intracranial encephalography, and action potential recording. The devices showed stable performance in physiological environments, being able to record signals for more than a week of implantation in a freely moving rodent. Finally, the E-IGTs showed remarkable advantages compared with conventional electrodes with silicon-based amplifiers; they present local amplification, they are composed of only biocompatible and commercially available materials, and they are flexible and compatible with a wide variety of plastic substrates.

Moreover, as explained in Section 3, the biocompatibility of films obtained from the commercial PEDOT:PSS solution is not adequate since the surface usually exhibits some of the features of the polyanion (such as negative charge), hampering its integration with biomolecules $[68,73]$. Therefore, different OECTs have been developed employing the above-mentioned biofunctionalization strategies.

For instance, Hai et al. developed an OECT for the label-free detection of human influenza A virus (Figure 11A,B) [119]. An electrodeposited film of poly(EDOTOA-coEDOT)/PEDOT:PSS was employed as semiconducting channel, and it was subsequently functionalized by glycosylation of 2,6-sialyllactose with oxylamine of the EDOTOA unit, a specific receptor for hemagglutinin, the spike protein on the surface of human influenza A virus (Figure 11C-E). The signal transduction mechanism involved the doping of the transistors due to the adsorption of negatively charged virus nanoparticles, which yielded changes in the recorded drain-source current. Moreover, the devices showed LOD values of two orders of magnitude lower than commercial immunochromatographic assays while exhibiting similar response time (Figure $11 \mathrm{~F}-\mathrm{H}$ ). 
A

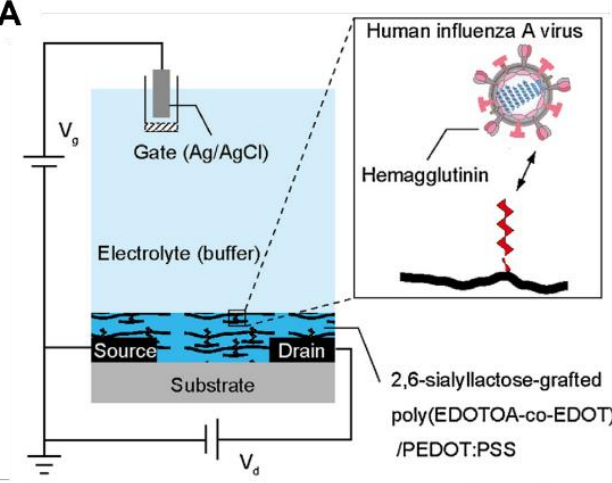

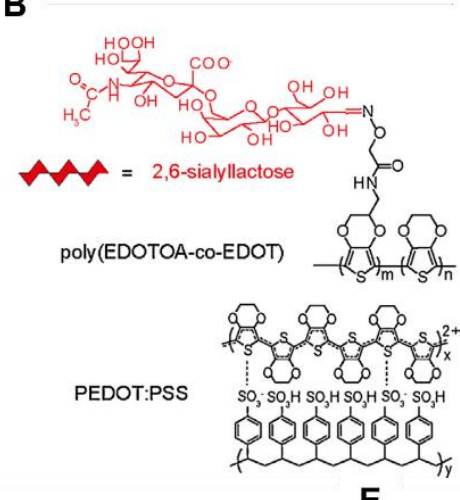

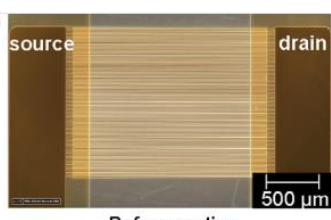

Before coating

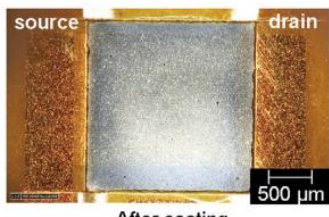

After coating

E
C

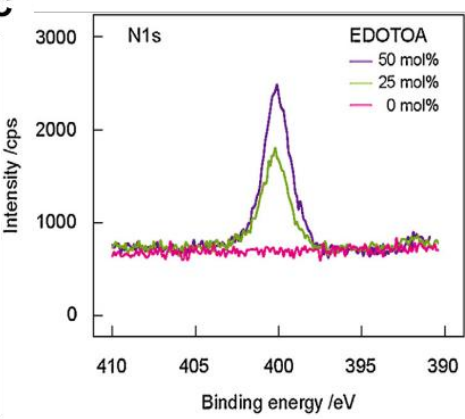

$\mathbf{F}$

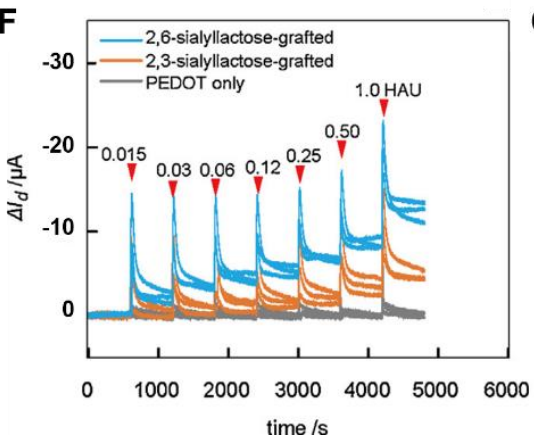

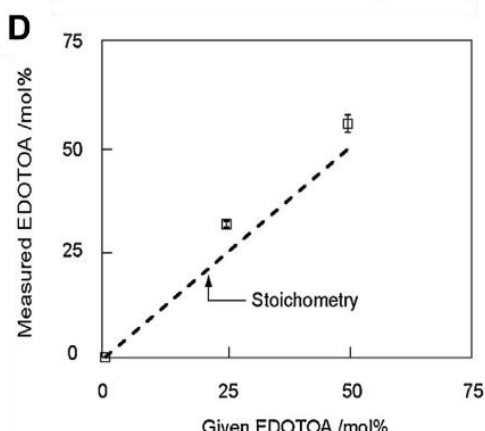

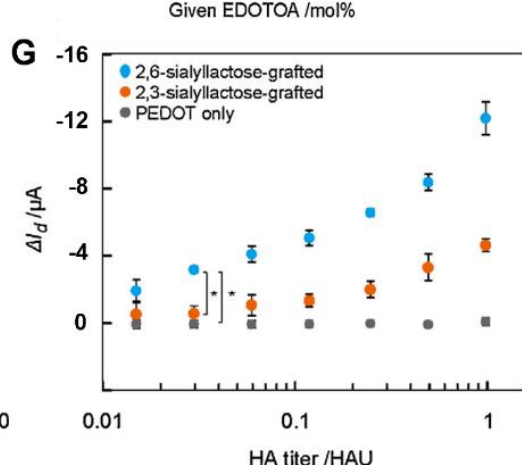

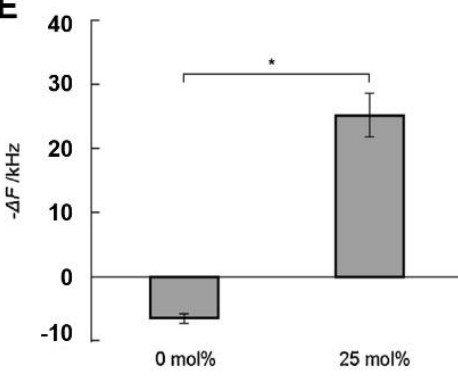

Given EDOTOA composition

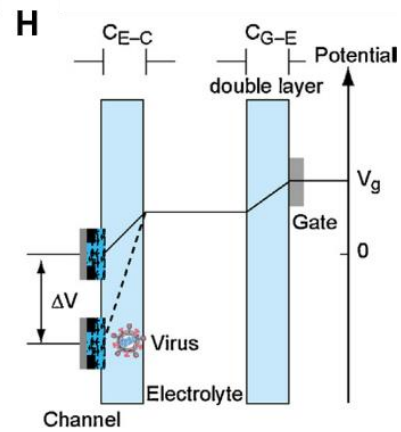

Figure 11. Scheme of the functionalized OECT (A). Chemical structures of 2,6-sialyllactose-grafted poly(EDOTOA-co-EDOT) and PEDOT:PSS and optical micrographs of the interdigitating microelectrodes before and after the coating of a PEDOT:PSS film (B). High-resolution XPS spectra of N1 s films at different compositions of EDOTOA during the electrodeposition on a PEDOT:PSS film (C). Given and measured compositions of EDOTOA on the surface of the electrodeposited film (D) Changes in the QCM frequencies before and after the reaction with 2,6-sialyllactose of PEDOT:PSS and poly(EDOTOAco-EDOT)/PEDOT:PSS films at $25 \mathrm{~mol} \%$ EDOTOA (E). Adapted with permission from reference [119]. Copyright 2020 Elsevier.

In this regard, when considering the fabrication of transistors-based biosensors, the immobilization of enzymes on amine-functionalized surfaces has been reported to be an effective approach $[71,120]$. Making use of this approach, Buth et al. modified the surface of OECTs of $\alpha$-sexithiophene (an analog of PEDOT without the dioxy substituent) with (3-aminopropyl)triethoxysilane (APTES) and showed that the acid-base equilibrium of the surface amino groups changed the doping state of the channel material, yielding an enhancement in the $\mathrm{pH}$ sensitivity of the devices [121]. Moreover, these moieties allowed for the anchoring of penicillinase via EDC-NHS chemistry, yielding penicillin biosensors with an estimated LOD of $5 \mu \mathrm{M}$.

Very recently, the fabrication of OECTs employing PEDOT:TOS and polyallylamine hydrochloride (PAH) composites as conducting channel material has been reported. To fabricate the devices, the polyelectrolyte was incorporated in the polymerization solution and subsequently casted onto interdigitated gold electrodes (Figure 12A). The regulation of the polyelectrolyte-to-conducting polymer proportion allowed for the straightforward tuning of both electronic and ionic characteristics of the devices, producing transistors with 
low threshold voltages while keeping high transconductance values, a crucial requirement for an efficient integration of OECTs with biological entities. Moreover, the integration of the polyelectrolyte in the conducting PEDOT matrix improved the transient response of the OECTs during the ON/OFF switching, most likely due to enhanced ion transport (Figure 12B). In addition, the $\mathrm{pH}$-sensitive amino moieties not only improved the $\mathrm{pH}$ response of the transistors but also enabled the non-denaturing electrostatic anchoring of functional enzymes. As a proof-of-concept, acetylcholinesterase was electrostatically anchored by taking advantage of the $\mathrm{NH}_{2}$ moieties, and the OECTs-based sensors were capable of detecting the neurotransmitter acetylcholine in the range 5-125 $\mu \mathrm{M}$ (Figure 12C) [91].

A
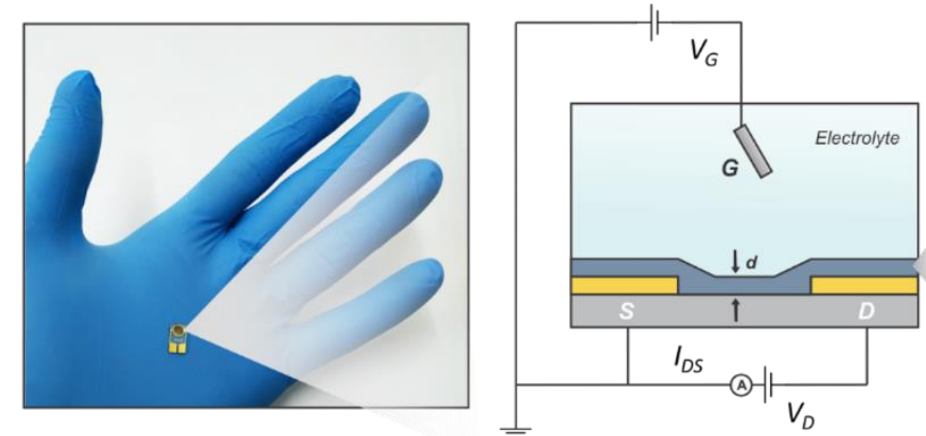

B

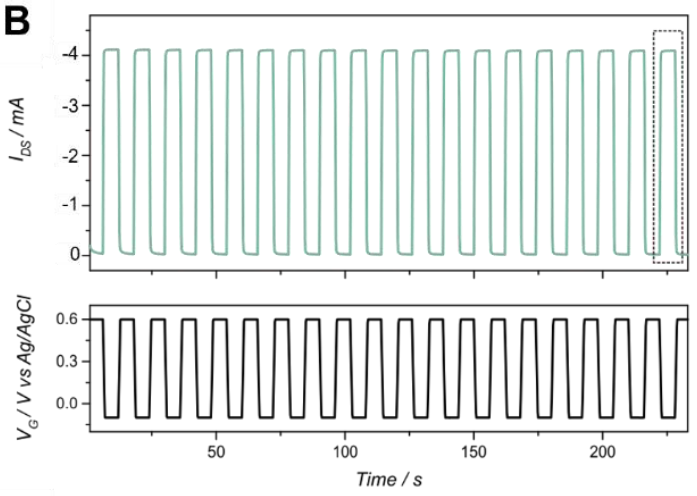

C

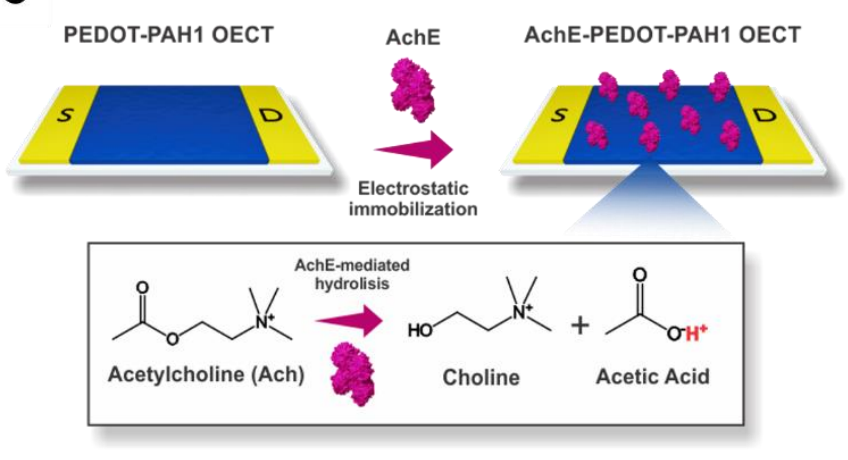

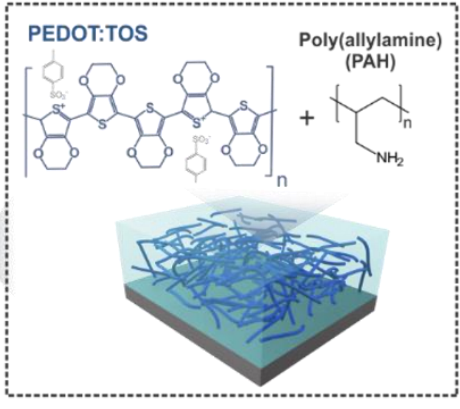
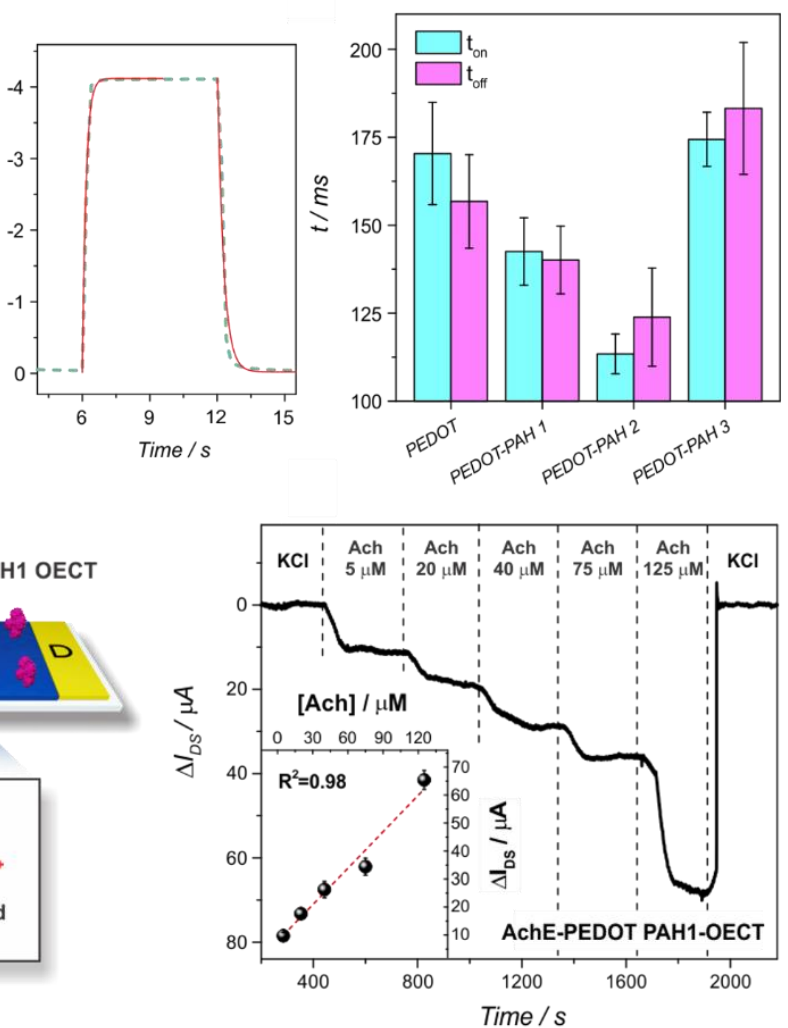

Figure 12. Scheme of a PEDOT-PAH OECT, measurement setup and representation of the polyelectrolyte-conducting polymer blend (A). Transient characteristics and exponential fitting for a PEDOT-PAH2 OECT (left) and switching ON and OFF times values obtained from the fitting for transistors fabricated employing different PAH-EDOT ratios (B). Scheme of the electrostatic immobilization of AchE and the Ach catalyzed hydrolysis (left), and Ach flow sensing experiment for an AchE-PEDOT-PAH1-OECT (right) (C). Adapted with permission from reference [91]. Copyright 2021 John Wiley and Sons.

By making use of the EDOT monomer derivatization strategy, Wustoni et al. reported the co-electropolymerization of films containing both EDOT and hydroxymethyl EDOT $(\mathrm{EDOTOH})$ monomers, yielding $\mathrm{p}(\mathrm{EDOT}-\mathrm{ran}-\mathrm{EDOTOH})$ in microscale transistor chan- 
nels [122]. The copolymer showed great charging capability and OECT performance. More importantly, the presence of hydroxyl moieties in the film enabled the stable incorporation of enzymes, yielding biologically functionalized transistors. Different enzymes such as GOx, lactate oxidase (LOx) and cholesterol oxidase (ChOx) were incorporated in the channel, and the miniaturized, multi-transistor platform was able to simultaneously detect glucose, cholesterol, and lactate.

In another interesting example, Carli et al. developed a water-based PEDOT:Nafion solution which was used for the preparation of different bioelectronics devices, namely OECTs, non-volatile memories (memristors) and artificial synapses [123]. The PEDOT:Nafion complex was prepared by solution-cast polymerization, and the different electronic devices were fabricated depositing a dilution of the as-synthesized PEDOT:Nafion solution on custom-designed test patterns. Different relevant features of PEDOT:Nafion were studied by physicochemical techniques and in the electronic device architectures, showing fast ion transport and stability to delamination, even in the absence of adhesion promoters. These results make PEDOT:Nafion a promissory material in the domain of printable organic electronics.

\subsection{Biosensing}

Biosensing is an interdisciplinary discipline which has shown great growth during recent decades, becoming one of the most active and dynamic areas in analytical chemistry research [124]. In this regard, biosensors can be considered to be complementary devices to traditional analytical methods due to their particular features, such as low cost, fast response time, and possibility of miniaturization, among others [70]. In particular, conducting polymers-based platforms have been extensively employed for the construction of biosensing devices as they can simultaneously offer a proper environment for the immobilization of the recognition elements and act as physicochemical transducer of the chemical signals into a measurable electrical response (or simply mediate the electron transfer to the electrode) [124-126]. Among them, and unlike PANI that requires acid media to exhibit good electrochemical activity [14,15], PEDOT shows excellent conductivity and electroactivity in neutral solution, which has motivated its widely extended application for the fabrication of biosensing platforms $[64,127]$.

However, when considering biosensors, the PEDOT film surface biofunctionalization is a necessary and critical step for the construction of efficient devices. Since the performance of the biosensor strongly depends on the features of the interfacial architecture, the recognition element (i.e., enzymes, antibodies, proteins) must be immobilized in a manner that ensures the preservation of its biological activity and the accessibility to its active sites [70,71]. Moreover, the biofunctionalization procedure must not affect the stability of the transducing component in order to establish an efficient charge transfer and/or conduction paths [128].

In this regard, multiple strategies have been developed to anchor different elements on PEDOT-based surfaces with the aim of developing efficient biosensing devices. For instance, Meng et al. have recently reported the preparation of a bifunctional nanofibrous PEDOT 3D network bearing carboxylic acid moieties (Nano-PEDOT-COOH) for the development of NADH and lactate biosensors. First, the electro-copolymerization of EDOT and EDOT-COOH was realized on glassy carbon electrodes employing tetrabutylammonium perchlorate, and the control of the EDOT:EDOT-COOH ratio allowed for the modulation of the diameter of the fibers together with the carboxylic acid group density. Interestingly, the nanofibers were assembled in a 3D network with high surface area, yielding low charge transfer resistance to the film and high transduction activity towards the co-enzyme NADH. Moreover, the carboxylic moieties enabled the anchoring of lactate dehydrogenase (LDH, a NADH-dependent dehydrogenase) via EDC-NHS chemistry, yielding a biosensor for lactate detection in the range of $0.05-1.8 \mathrm{mM}$ with a response time lower than $10 \mathrm{~s}$ [129].

Click chemistry has also been shown to be a useful strategy for the development of PEDOT-based biosensors. For instance, Galán et al. have recently reported the fabrication of 
a label-free electrochemical DNA sensor based on azidomethyl-substituted (azido)-PEDOT electrodes making use of click chemistry (Figure 13) [74]. First, the electropolymerization of azido-EDOT in dichloromethane was performed on Au electrodes under argon atmosphere. Next, an acetylene-terminated DNA probe, complementary to a specific "Hepatitis C" virus sequence, was immobilized to azido-PEDOT using "click" chemistry. Finally, DNA hybridization was detected by differential pulse voltammetry (DPV), showing a LOD of $0.13 \mathrm{nM}$

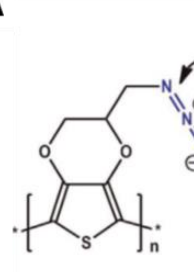

Azide-PEDOT

C

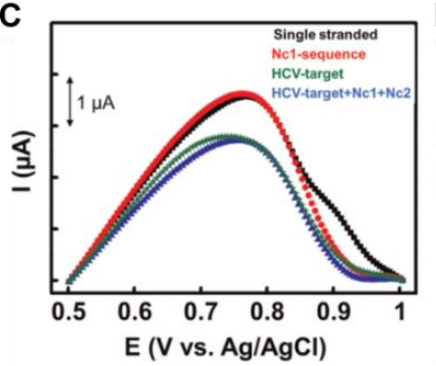

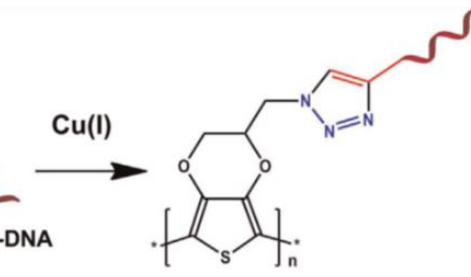

DNA functionalized PEDOT

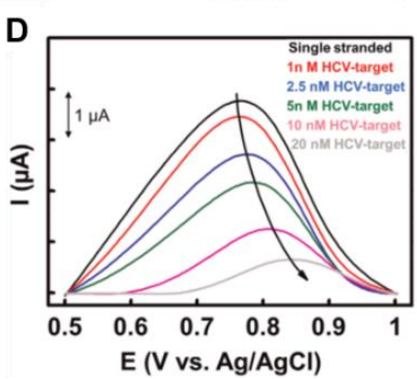

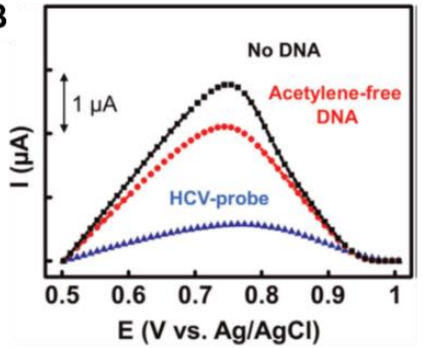

E

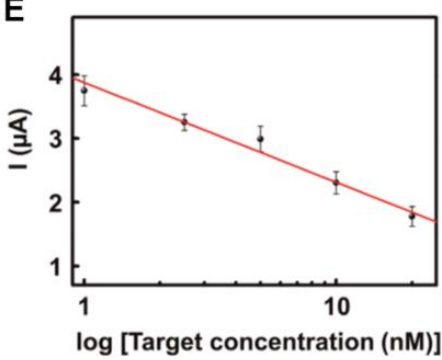

Figure 13. Scheme of the fabrication of DNA immobilization process by "click" reaction (A). DPV measurements after "click" reaction (B). Selectivity evaluation of the DNA sensor by DPV measurements $(\mathbf{C})$ and corresponding calibration curve $(n=3)(\mathbf{D})$. Adapted with permission from reference [74]. Copyright 2015 Elsevier.

Moreover, when considering the biosensing field, the development of flexible electrochemical platforms has received remarkable interest from the scientific community over the last decade due to their great potential to build implantable devices for predictive analyses and personalized medicine [130-132]. In this regard, while the preparation of PEDOTbased films on metal electrodes have been widely reported, this substrates are not suitable when it comes to the development of flexible platforms, making necessary the employment of conductive surfaces which also show mechanical stability [2,133]. Here is where PEDOT and PEDOT-related materials offer their very interesting properties, showing excellent conductivity as well as light weight, high flexibility, and mechanical adaptability $[16,68]$. Therefore, several strategies have been developed in order to allow the biofunctionalization of PEDOT and PEDOT-like flexible electrodes [134].

For instance, employing a strategy previously described, Sappia et al. have developed flexible all-polymer biosensing platforms based on PEDOT-PAH films (Figure 14A) [90]. The polyamine-PEDOT blend was deposited on flexible PET films, showing a better mechanical performance than pristine PEDOT (Figure 14B). Moreover, the incorporation of amino residues on the film allowed the covalent anchoring of sugar motifs by employing divinylsulfone (DVS) chemistry, and, by thus, the further immobilization of lectins and glycoenzymes mediated by carbohydrate-lectin recognition. The strategy initially involved the incubation of the polyamine biosensors in a 5\% DVS solution in carbonate buffer. Next, the films were glycosylated either with mannose or N-acetylglucosamine (GlcNAc) in the same buffer. Subsequently, the self-assembly of Concanavalin A (ConA) and Wheat germ agglutinin (WGA) lectins on the glycosylated (mannose or $\mathrm{N}$-acetylglucosamine, respectively) PEDOT-PAH films was performed in HEPES buffer, as show in Figure 14C). Moreover, the ConA integration further allowed for the assembly of two model glycoenzymes (GOx and HRP) via biorecognition interactions, and the further electrochemical 
detection of glucose and hydrogen peroxide by the flexible all-polymer biosensors. The authors found that biosensors fabricated employing thinner polymer layers showed better glucose sensing performance, probably due to a diminished capacitive contribution from the thicker conducting polymer films (Figure 14D). Furthermore, the PEDOT-PAH platforms without the ConA immobilization step showed negligible non-specific GOx adsorption, confirming the specificity of the developed approach.
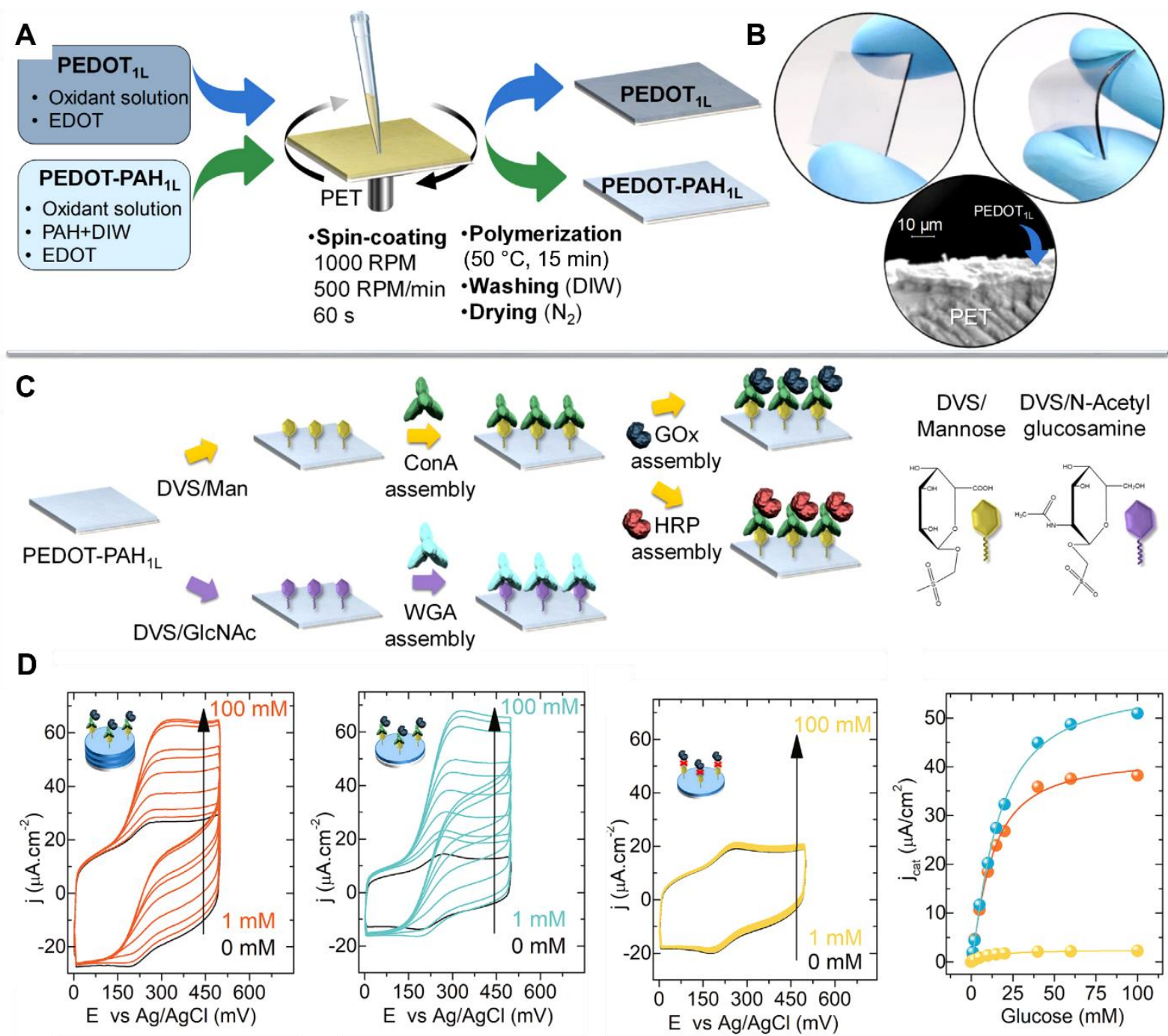

Figure 14. Scheme for the fabrication of PEDOT and PEDOT-PAH1L films by spin-coating on PET foils (A). Images of a flexible PET foil substrate modified with film of PEDOT-PAH (B). Sequential functionalization of the PEDOT-PAH composite (C). Cyclic voltammograms and current densities of the glucose oxidation for Con A/GOx-functionalized films prepared with different PEDOT-based layers (red, 1 layer of PEDOT-PAH, light blue, 2 PEDOT layers+1 layer of PEDOT-PAH). The yellow curves correspond to the PEDOT-PAH electrode without ConA (D). Adapted with permission from reference [90]. Copyright 2020 Elsevier.

Very recently, Xu et al. have developed a flexible, fully organic, biodegradable, labelfree impedimetric biosensor for the detection of the vascular endothelial growth factor (VEGF) biomarker [135]. To fabricate the biosensors, a conducting ink containing PEDOT:PSS, anti-VEGF165 antibody and photoreactive sericin was spin-coated and patterned onto previously obtained fibroin substrates. Afterwards, the films were detached from the glass support, yielding free-standing platforms. The developed biosensor was demonstrated to be highly sensitive and selective to the target protein, performing the impedimetric detection in a range of biologically relevant fluids such as buffer, serum, and urine. 
Moreover, the platform was demonstrated to be mechanically compliant and conformable to soft tissue, as well as biocompatible and degradable.

Similarly, Pal et al. reported a facile approach employing photolithography in order to fabricate PEDOT:PSS-based glucose sensors on a fully biodegradable and flexible silk protein fibroin support [136]. High-resolution PEDOT:PSS microstructures were fabricated over a large area using water as solvent and were demonstrated to retain the electrochemical activity up to 3 days of PBS soaking. Moreover, the electrodes were able to detect dopamine and ascorbic acid with high sensitivity. In addition, the conducting ink was able to function as matrix for enzymes, enabling the detection of glucose. The strategy constitutes a valuable approach towards the development of mechanically flexible, transparent, electroactive, cytocompatible and biodegradable devices.

\subsection{Electrophysiological Recording}

The recording of physiological signals is key to evaluate the physiological state of patients and healthy individuals. Moreover, the continuous monitoring enables pointof-care disease diagnostics and allows healthy persons to profit from it as preventive medicine while continuing their daily lives [137]. For instance, among these signals, the recording of cardiac activity by electrocardiography (ECG) is crucial to detect abnormalities such as tachycardia or myocardial infarction [138]. Similarly, the recording of muscle activity by surface electromyography (EMG) is employed as a medical diagnostic and to control prosthetic members. Finally, electroencephalography (EEG) and neural recordings provide information about neurological rhythms as well as the oscillations of small cell clusters, activity of single neurons, and subthreshold activity throughout various brain circumstances $[139,140]$.

Next, to allow for effective recordings of those signals, the electrodes need to fulfill specific requirements, such as having an adequate contact with the body without presenting toxicity or triggering allergic reactions, having low cost, and showing comfortable wearing. When considering the quality of the recordings, to show low impedance at the electrode/skin interface is also a must for the devices [140,141]. In this regard, organic PEDOT-based electrodes (and, particularly, PEDOT:PSS-based) have replaced traditional metal substrates due to their low impedance, availability, flexibility, and biocompatibility $[142,143]$. For instance, a low-cost, dry, imperceptible, and temporary PEDOT:PSS-based tattoo electrode able to perform surface electromyography and electrocardiography recordings on various locations on limbs and face was recently reported by Ferrari et al. [144].

Despite the great advances in this field, there is still room from improving those electrodes, especially when it comes to the biocompatibility of the material and its physical and electronic features, such as flexibility and charge injection properties. Therefore, different strategies have been developed to create more efficient electrophysiological recording devices. For instance, and very recently, Velasco-Bosom et al. reported the fabrication of scalable electrode arrays comprised of composites of PEDOT:PSS and the biocompatible ionic liquid cholinium which were able to capture electromyography signals with millimeter spatial resolution (Figure 15) [145]. The devices were fabricated through inkjet-printing and laser-cutting fabrication techniques (Figure 15A-C) and enabled the recording of highquality spatiotemporal signals from the forearm of individuals. These recordings allowed for the identification of the motions of the index, little, and middle fingers, and the direct visualization of the propagation of polarization/depolarization waves in the underlying muscles (Figure 15D-F). 


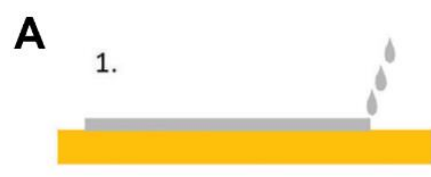

2.
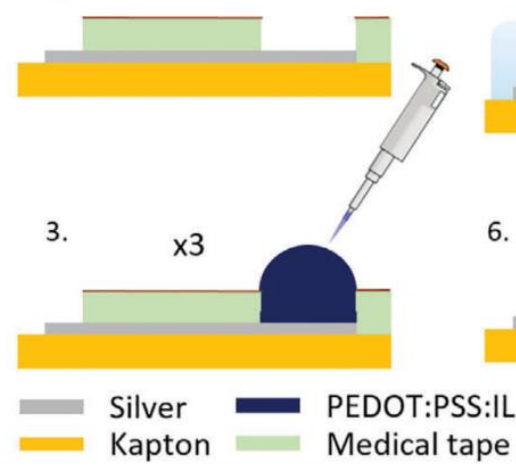

4.

5.
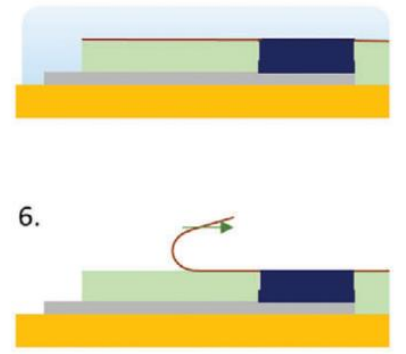

Liner
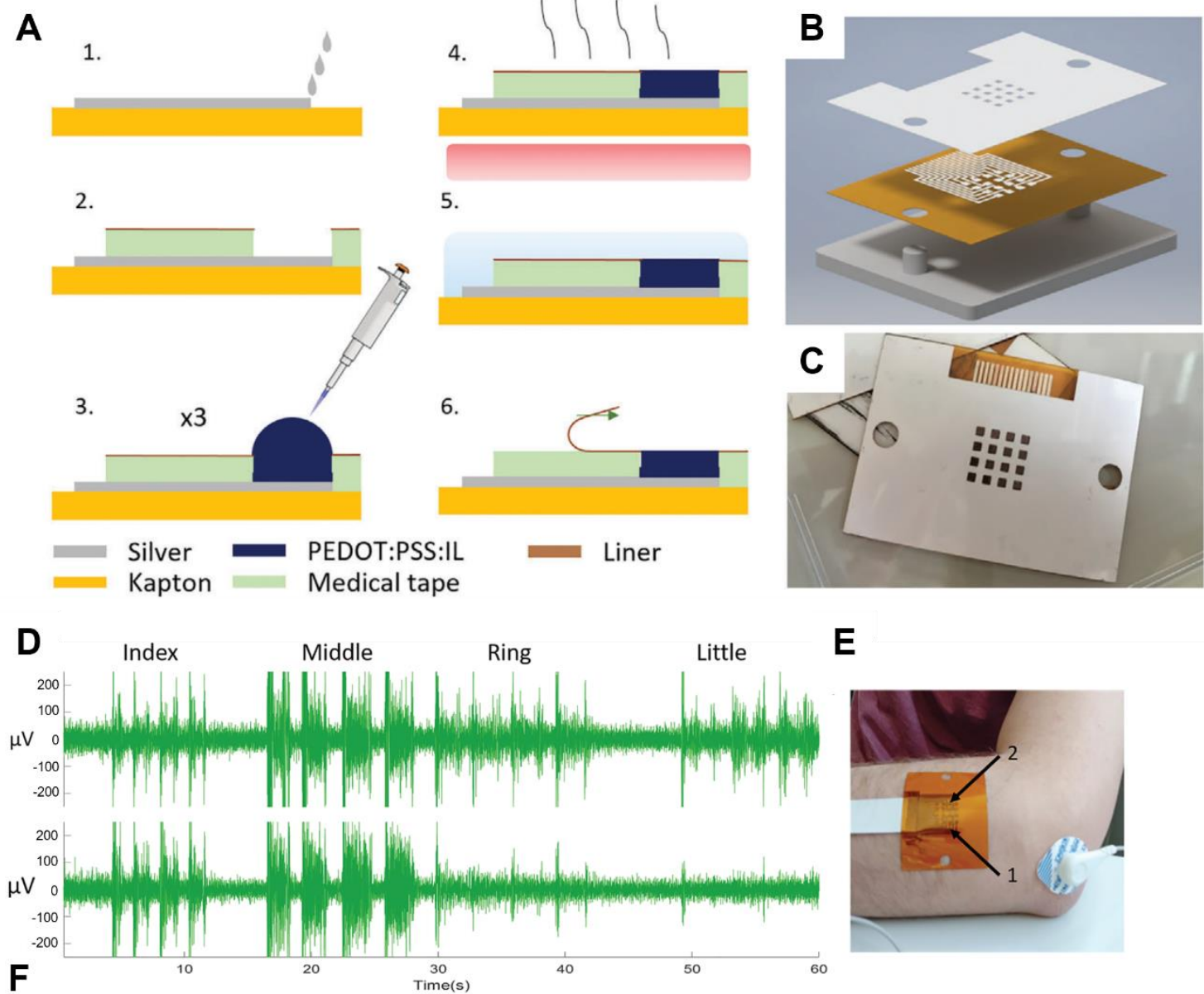

E

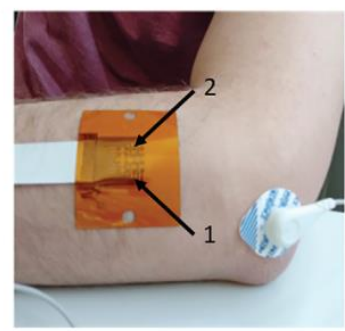

Little finger

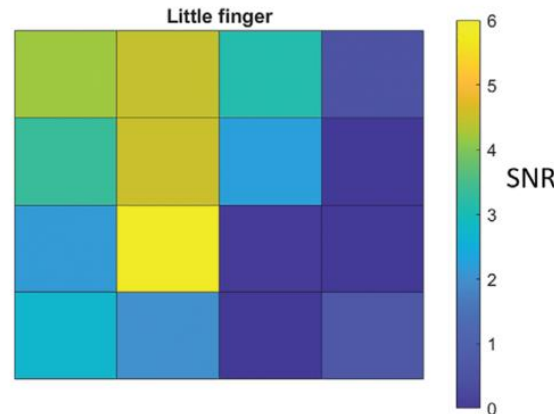

Figure 15. Scheme of the fabrication process of the electrode arrays (A). Schematic view of the assembly process of the device showing the aligning holder (B). Photo of a device before measuring (C). Typical EMG recordings from two electrodes during the motion of different fingers (D). Photo showing the location of the electrode array and the reference electrode. The arrows indicate the location of the two electrodes, over the extensor carpi ulnaris (1) and the extensor digitorum communis (2) muscles (E). Heat maps showing the normalized signals recorded by the array during the motion of different fingers (F). Adapted under the terms of the CC-BY-4.0 license [145]. Copyright 2021, The author(s).

Another interesting approach involves the deposition of PEDOT films employing Nafion. For instance, the electrodeposition of PEDOT:Nafion films on microelectrode arrays was recently reported, as well as its comparison with PEDOT:PSS films in terms of electrochemical, morphological, and in vivo electrophysiological features [146]. PEDOT:Nafion exhibited a similar performance compared with PEDOT:PSS in terms of neural recording, while the charge injection limit was two times larger. In a similar fashion, Vreeland et al. reported the electropolymerization of a Nafion/PEDOT composite polymer on carbon-fiber microelectrodes with the goal of creating a mechanically stable, robust, and controllable electrode coating that increases the selectivity and sensitivity for in vivo electrochemical 
measurements. PEDOT:Nafion-coated electrodes were lowered into the nucleus accumbens of a rat, and both spontaneous and electrically evoked dopamine release were measured. In addition to improvements in sensitivity and selectivity, the coating dramatically reduced acute in vivo biofouling [147].

In another study, del Agua et al. reported the fabrication of conducting, water stable, free-standing textile electrodes by adding the crosslinker divinyl sulfone (DVS) to PEDOT:PSS formulations [137]. The addition of DVS enables the preparation of low resistance free-standing as well as textile electrodes without hampering the conductivity of the films nor its mechanical features. Later, both electrodes were evaluated for their application towards cutaneous electrophysiology (particularly ECG) showing great performance and allowing for precise electrophysiological recordings in a comfortable way.

\subsection{Cell Interfacing and Tissue Engineering}

The interaction of materials with cells (and, in a larger scale, with tissues) is key in a wide range of applications such as neuronal activity monitoring, stimulation devices, scaffolds for tissue regeneration, drug delivery, and cell culture [72]. Therefore, the rational functionalization of surfaces for improving these interactions and/or promote a specific cell behavior, has received growing interest from the scientific community in recent years [140]. In this regard, the organic nature of PEDOT-based platforms enables biocompatible surfaces possessing particular electric and optical properties. PEDOT films possess an exceptional feature when exchanging signals with cells, as they are able of transducing electric to ionic signals and vice versa [148]. This feature becomes particularly relevant in applications requiring communication through interfaces as those happening between cells and substrates, allowing the fabricated devices to monitor neuronal activity, simulate nerve impulses or perform controlled drug delivery [149]. Thus, PEDOT-based substrates for cell culture are able to electrically stimulate cells in order to promote growth or incite differentiation [150].

In this regard, the functionalization of PEDOT-based surfaces with biomolecules is necessary to improve cell-surface interactions. In particular, proteins are essential players in this interaction, and their nature, orientation and conformation are crucial for the development of an effective communication across the different interfaces [151]. Among them, integrins are a relevant type of cell adhesive glycoproteins which can recognize specific peptide sequences in extracellular matrix proteins such as laminin, collagen, and fibronectin. Hence, their incorporation in the composite matrix has been shown to be an interesting approach to develop efficient cell interacting systems [151,152].

In an interesting approach, Maione et al. reported the preparation of electroactive films based on PEDOT and an Arg-Gly-Asp (RGD)-like peptide [153]. First, the authors performed the synthesis of a peptide where Gly was substituted by an amino acid bearing the EDOT moiety $\left(R G^{\mathrm{E}} \mathrm{D}\right)$ in the side chain. Later, the preparation of conducting films based on $\mathrm{RG}^{\mathrm{E}} \mathrm{D}$ was performed by electropolymerization, either using EDOT as a co-monomer or not, onto previously PEDOT-modified steel electrodes. The effect of the peptide incorporation increased the wettability of the obtained films for both employed strategies, while the surface roughness only increased for the copolymerization strategy. Furthermore, it was shown that the incorporation of $\mathrm{RG}^{\mathrm{E}} \mathrm{D}$ negatively affects the electrochemical stability of the films, probably due to the degradation of the peptide during the oxidation and reduction processes. Finally, and due to the cell recognition abilities of the RGD motif, the electroactive surfaces prepared using the conjugates displayed higher bioactivities in terms of cell adhesion, and the relative viabilities depended on the wettability, roughness, and electrochemical activity of the surface.

The incorporation of acid moieties has been also shown to improve PEDOT-interfacing properties. For instance, Sekine et al. have recently reported the fabrication of a PEDOT$\mathrm{COOH}$-based nanostructured platform in order to improve cell interfacing and capturing [154]. First, PEDOT-COOH films presenting different nanodots size and density were obtained on ITO substrates by electropolymerization. Later, and since most tumor cells 
overexpress a membrane antigen named the epithelial cell-adhesion molecule (EpCAM), the PEDOT-COOH nanodots films were conjugated with a capturing agent, antihuman EpCAM (anti-EpCAM), to test their capture efficiency. In this regard, the carboxylic acid moieties were first conjugated with streptavidin via EDC-NHS chemistry and subsequently incubated with biotinylated anti-EpCAM (Figure 16A). Finally, the interaction of the different PEDOT-functionalized films with five tumor cell lines of interest for circulating tumor cell diagnostics was evaluated, demonstrating that the efficiency of specific cell capture by EpCAM antibodies was enhanced by four to five times compared to PEDOT-COOH smooth films. (Figure 16B-E).

A
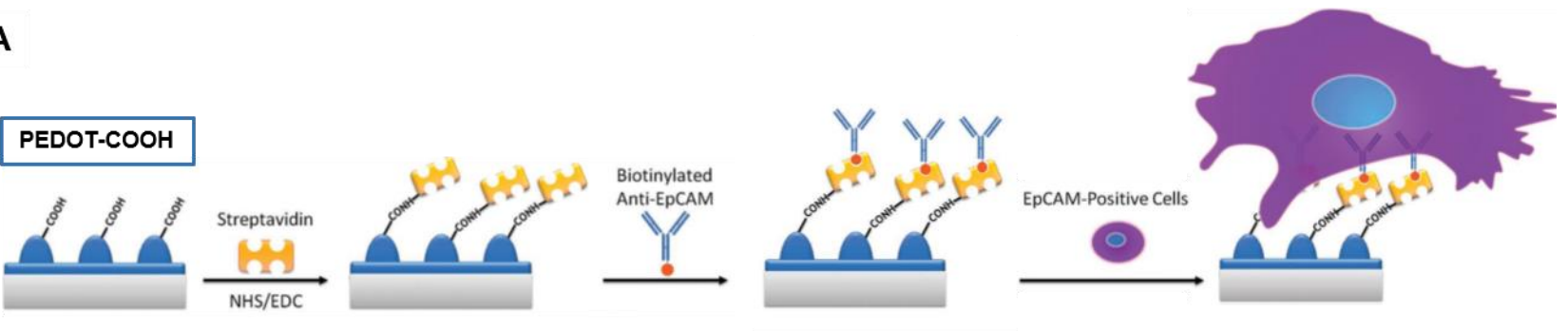

B
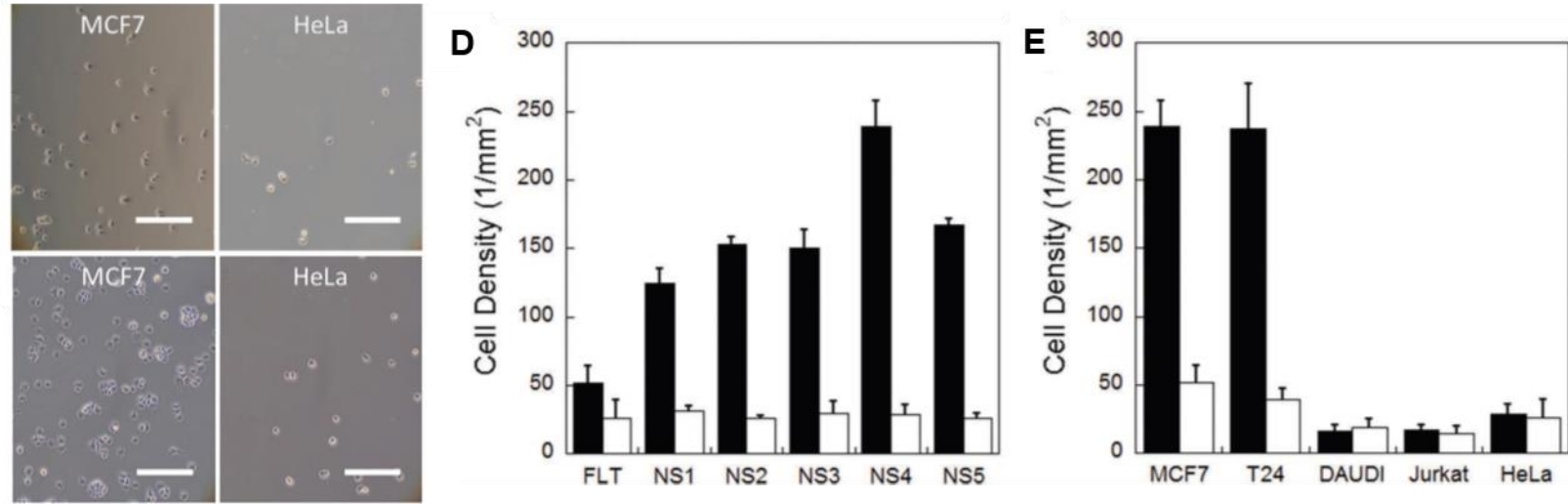

Figure 16. Scheme of the PEDOT-COOH functionalization process (A). MCF7 and HeLa cell capturing using flat antiEpCAM conjugated PEDOT films (PEDOT-FLT) (B) and anti-EpCAM conjugated PEDOT nanodots (PEDOT-NS4) (C). MCF7 and HeLa cells capture yields for different anti-EpCAM conjugated PEDOT films (D). Synergistic effect of morphology and capture agents on cell capturing. Capture yields of various cell lines using anti-EpCAM conjugated PEDOT nanodots PEDOT-NS4 (black) and PEDOT films PEDOT-FLT (white). MCF7 and T24 cells are EpCAM-(+). DAUDI, Jurkat, and HeLa cells are EpCAM-(-) (E). Adapted with permission from reference [154]. Copyright 2011 John Wiley and Sons.

Although flat surfaces can be useful to study particular interactions, sometimes a mechanical support which offers a more similar environment to the extracellular matrix is needed to obtain a more "real" behavior of the cells. In this regard, PEDOT-related materials have been also employed for the fabrication of scaffolds for cell culture [2,155]. In particular, a very effective approach involves the incorporation of hydrogel networks in the conducting PEDOT matrix. For instance, Huang et al. have recently developed a conductive PEDOT-chitin hydrogel that served as nerve guidance conduit [156]. First, the polysaccharide chitin was employed to fabricate the hydrogel film and its mechanical features were improved by partial deacetylation. The porous structure of the hydrogel together with its enhanced biocompatibility considerably stimulated nerve cell attachment and proliferation in vitro, while cell electrical stimulation promoted nerve cell-related gene expression. Finally, in vivo studies showed rats sciatic nerve repair after the incorporation of the PEDOT hydrogel, probably due to the enhancement of the adhesion of Schwann cells and the angiogenesis at the injury sites.

$\mathrm{Hu}$ et al. reported the fabrication of a conducting hydrogel based on PEDOT:PSS and peptide-modified PEG through a noncovalent assembling approach [157]. The developed 
strategy enabled the modulation of the mechanical and electronic properties of the system through the control of the synthesis conditions. Moreover, the conducting network presented self-healing properties and supported the survival and growth of cells. Furthermore, the assembled matrix enabled the development of 3D-cell cultures with incorporated electrical stimulation (ES) function, as shown by the support of the survival and proliferation of encapsulated mesenchymal stromal cells and their differentiation under ES.

In another interesting example, Spencer et al. reported the synthesis of a conducting matrix based on PEDOT:PSS dispersed within a photo-crosslinkable hydrogel, gelatin methacryloyl (GelMA) [158]. To fabricate the hydrogels, different PEDOT:PSS amounts were added to GelMA solutions, and the crosslinking was subsequently performed. The effect of PEDOT:PSS loading on the electrical and mechanical features of the hydrogels, and on their degradation, swelling, and biocompatibility was studied. It was shown that the incorporation of PEDOT:PSS lowered the impedance of the matrix at physiologically relevant frequencies and allowed the excitation of explanted abdominal muscle tissue. Finally, in vitro studies showed that hydrogels containing $0.1 \%(w / v)$ PEDOT:PSS sustained the viability and propagation of $\mathrm{C} 2 \mathrm{C} 12$ myoblasts, showing a performance comparable to that of GelMA structures.

\subsection{Overview}

From the literature reviewed in this section it is possible to derive more general observations. First, when considering the fabrication of OECTs, it is observed that PEDOT:PSS is probably the most employed material for the fabrication of those devices. In this regard, the commercial availability together with the straightforward and reproducible deposition on a wide variety of substrates of the PEDOT:PSS dispersion drives its widespread use for the development of organic transistors. However, as previously stated, the functionalization of this polymer is a must to develop efficient organic electronic devices. Therefore, the addition of molecules to the EDOT polymerization solution is also reported as an efficient method to fabricate OECTs, and the incorporation of polyamines appears as one of the most employed strategies inside this approach. On the other hand, the electropolymerization of derivatized monomers is not so widely employed for the fabrication of the conducting channel of OECTs, probably due to the different reactivity rates of the derivatized monomers and the requirement of reproducible polymerization conditions between both source and drain electrodes. However, few advances have been recently reported, and we envisage a growth of the field in this direction.

On the other hand, PEDOT:PSS is not so widely employed for the development of usual three electrode-based biosensing platforms, probably due to the low bio-functionality of the polymer. In this regard, when conducting substrates are employed, the electropolymerization of derivatized EDOT monomers appears as an efficient technique for the development of reproducible and reliable biosensing devices. However, when a flexible biosensing device is desired, the obtention of a conducting platform cannot be realized by this approach, and the incorporation of additives to the PEDOT polymerization solution is chosen to improve the biocompatibility of the surface, allowing the further anchoring of different recognition elements without dramatically affecting the conductivity.

Regarding the applications related to cell interfacing and tissue engineering, the functionalization of PEDOT with proteins (or, at least, peptides) is a requirement for the improvement of the interaction of the film with the cells. Therefore, the obtention of functionalized PEDOT by electropolymerization is reported to be an efficient approach since the amount of the biological element incorporated can be easily controlled. Moreover, and to match the mechanical features of the films with those of the cells, hydrogel-PEDOT combined matrixes are being increasingly employed to fabricate films presenting improved interfacing features, as well as better compatibility with different tissues.

Finally, for the efficient recording of electrophysiological signals, not only the mechanical features of the film should be matched with those of the target tissue, but also the platform must show a low impedance to efficiently transduce the electronic signal. In this 
sense, PEDOT:PSS shows very interesting features, driving its widespread use towards this particular application. Nevertheless, its functionalization towards the generation of a more compatible interface results necessary.

\section{Summary and Outlook}

In summary, we have discussed several functionalization strategies for PEDOT and PEDOT:PSS films to incorporate a wide range of biomolecules for bioelectronics devices. Since the election of these approaches will greatly depend on the film preparation method, we first discussed PEDOT and PEDOT:PSS films synthesis techniques. Next, a wide range of functionalization approaches were presented. The incorporation and tuning of different functional moieties on PEDOT were shown to enable the further integration of a wide variety of biomolecules, such as proteins and peptides, as well as biorecognition entities including nucleic acids, antibodies, and enzymes. In the last section, we showed how these biofunctionalized PEDOT surfaces can be employed towards the generation of platforms showing very relevant applications.

Finally, while this field continues to progress further and further every day, the development of new functionalization approaches is necessary for the improvement of the present biosensing and biomedical devices based on PEDOT, as well as for the emergency of new technological applications. New advances in the field will surely be achieved coming from the synergistic efforts by both organic semiconductors and biotechnological researchers. We believe that the development of bioelectronic devices will be directed towards the fabrication of wearable units able to monitor different simultaneous physiological signals in real time. In this regard, and helped by the integration of energy storage devices, the transduction of bioelectronic signals such as biomarkers concentration and electrophysiological recordings could be easily and reliably performed by these devices by taking advantage of the soft nature and the high conductivity of PEDOT and PEDOT:PSS-based platforms. In this regard, the occurrence of a proper tissue-platform interfacial connection is a must. Finally, the development and commercialization of these point-of-care and mobile health technologies will simplify the health monitoring of patients, allowing more patients/individuals to be supervised.

Author Contributions: Conceptualization, G.E.F., W.A.M., O.A. and W.K.; investigation, G.E.F. and W.A.M.; data curation, G.E.F. and W.A.M.; writing—original draft preparation, G.E.F., W.A.M. and O.A.; writing-review and editing, G.E.F., W.A.M., O.A. and W.K.; supervision, W.A.M.; project administration, W.A.M. and O.A.; funding acquisition, W.A.M., W.K. and O.A. All authors have read and agreed to the published version of the manuscript.

Funding: This research was funded by ANPCyT (PICT-2017-1523, PICT-2018-0780), CEST-Competence Center for Electrochemical Surface Technologies (CEST-UNLP Partner Lab for Bioelectronics) and Universidad Nacional de La Plata (PID-X867).

Institutional Review Board Statement: Not applicable.

Informed Consent Statement: Not applicable.

Data Availability Statement: Not applicable.

Acknowledgments: G.E.F. acknowledges CONICET for a fellowship.

Conflicts of Interest: The authors declare no conflict of interest.

\section{References}

1. Simon, D.T.; Gabrielsson, E.O.; Tybrandt, K.; Berggren, M. Organic Bioelectronics: Bridging the Signaling Gap between Biology and Technology. Chem. Rev. 2016, 116, 13009-13041. [CrossRef] [PubMed]

2. Zeglio, E.; Rutz, A.L.; Winkler, T.E.; Malliaras, G.G.; Herland, A. Conjugated Polymers for Assessing and Controlling Biological Functions. Adv. Mater. 2019, 1806712, 1806712. [CrossRef] [PubMed]

3. Smela, E. Conjugated polymer actuators for biomedical applications. Adv. Mater. 2003, 15, 481-494. [CrossRef] 
4. Promsuwan, K.; Meng, L.; Suklim, P.; Limbut, W.; Thavarungkul, P.; Kanatharana, P.; Mak, W.C. Bio-PEDOT: Modulating Carboxyl Moieties in Poly(3,4-ethylenedioxythiophene) for Enzyme-Coupled Bioelectronic Interfaces. ACS Appl. Mater. Interfaces 2020, 12, 39841-39849. [CrossRef] [PubMed]

5. Ludwig, K.A.; Langhals, N.B.; Joseph, M.D.; Richardson-Burns, S.M.; Hendricks, J.L.; Kipke, D.R. Poly(3,4-ethylenedioxythiophene) (PEDOT) polymer coatings facilitate smaller neural recording electrodes. J. Neural Eng. 2011, 8, 014001. [CrossRef]

6. Rivnay, J.; Inal, S.; Salleo, A.; Owens, R.M.; Berggren, M.; Malliaras, G.G. Organic electrochemical transistors. Nat. Rev. Mater. 2018, 3, 17086. [CrossRef]

7. Macchia, E.; Manoli, K.; Di Franco, C.; Picca, R.A.; Österbacka, R.; Palazzo, G.; Torricelli, F.; Scamarcio, G.; Torsi, L. Organic Field-Effect Transistor Platform for Label-Free, Single-Molecule Detection of Genomic Biomarkers. ACS Sens. 2020, 5, 1822-1830. [CrossRef]

8. Strakosas, X.; Bongo, M.; Owens, R.M. The organic electrochemical transistor for biological applications. J. Appl. Polym. Sci. 2015, 132, 1-14. [CrossRef]

9. Berggren, M.; Richter-Dahlfors, A. Organic bioelectronics. Adv. Mater. 2007, 19, 3201-3213. [CrossRef]

10. Zeglio, E.; Inganäs, O. Active Materials for Organic Electrochemical Transistors. Adv. Mater. 2018, 30, 1-18. [CrossRef]

11. Fenoy, G.E.; Van der Schueren, B.; Scotto, J.; Boulmedais, F.; Ceolín, M.R.; Bégin-Colin, S.; Bégin, D.; Marmisollé, W.A.; Azzaroni, O. Layer-by-layer assembly of iron oxide-decorated few-layer graphene/PANI:PSS composite films for high performance supercapacitors operating in neutral aqueous electrolytes. Electrochim. Acta 2018, 283, 1178-1187. [CrossRef]

12. Berggren, M.; Crispin, X.; Fabiano, S.; Jonsson, M.P.; Simon, D.T.; Stavrinidou, E.; Tybrandt, K.; Zozoulenko, I. Ion ElectronCoupled Functionality in Materials and Devices Based on Conjugated Polymers. Adv. Mater. 2019, 1805813, 1-15. [CrossRef]

13. Keene, S.T.; van der Pol, T.P.A.; Zakhidov, D.; Weijtens, C.H.L.; Janssen, R.A.J.; Salleo, A.; van de Burgt, Y. Enhancement-Mode PEDOT:PSS Organic Electrochemical Transistors Using Molecular De-Doping. Adv. Mater. 2020, 32. [CrossRef]

14. Fenoy, G.E.; Scotto, J.; Azcárate, J.; Rafti, M.; Marmisollé, W.A.; Azzaroni, O. Powering Up the Oxygen Reduction Reaction through the Integration of $\mathrm{O}_{2}$-Adsorbing Metal-Organic Frameworks on Nanocomposite Electrodes. ACS Appl. Energy Mater. 2018, 1, 5428-5436. [CrossRef]

15. Fenoy, G.E.; Giussi, J.M.; von Bilderling, C.; Maza, E.M.; Pietrasanta, L.I.; Knoll, W.; Marmisollé, W.A.; Azzaroni, O. Reversible modulation of the redox activity in conducting polymer nanofilms induced by hydrophobic collapse of a surface-grafted polyelectrolyte. J. Colloid Interface Sci. 2018, 518, 92-101. [CrossRef] [PubMed]

16. Gueye, M.N.; Carella, A.; Faure-Vincent, J.; Demadrille, R.; Simonato, J.-P. Progress in understanding structure and transport properties of PEDOT-based materials: A critical review. Prog. Mater. Sci. 2020, 108, 100616. [CrossRef]

17. Jiang, Y.; Liu, T.; Zhou, Y. Recent Advances of Synthesis, Properties, Film Fabrication Methods, Modifications of Poly(3,4ethylenedioxythiophene), and Applications in Solution-Processed Photovoltaics. Adv. Funct. Mater. 2020, 2006213, 1-46. [CrossRef]

18. Pérez-Mitta, G.; Marmisollé, W.A.; Trautmann, C.; Toimil-Molares, M.E.; Azzaroni, O. An All-Plastic Field-Effect Nanofluidic Diode Gated by a Conducting Polymer Layer. Adv. Mater. 2017, 29, 1700972. [CrossRef]

19. Ohayon, D.; Inal, S. Organic Bioelectronics: From Functional Materials to Next-Generation Devices and Power Sources. Adv. Mater. 2020, 32, 1-29. [CrossRef]

20. Groenendaal, L.; Zotti, G.; Aubert, P.H.; Waybright, S.M.; Reynolds, J.R. Electrochemistry of poly(3,4-alkylenedioxythiophene) derivatives. Adv. Mater. 2003, 15, 855-879. [CrossRef]

21. Strakosas, X.; Wei, B.; Martin, D.C.; Owens, R.M. Biofunctionalization of polydioxythiophene derivatives for biomedical applications. J. Mater. Chem. B 2016, 4, 4952-4968. [CrossRef]

22. Minudri, D.; Mantione, D.; Dominguez-Alfaro, A.; Moya, S.; Maza, E.; Bellacanzone, C.; Antognazza, M.R.; Mecerreyes, D. Water Soluble Cationic Poly(3,4-Ethylenedioxythiophene) PEDOT-N as a Versatile Conducting Polymer for Bioelectronics. Adv. Electron. Mater. 2020, 6, 1-10. [CrossRef]

23. Hermanson, G.T. Bioconjugate Techniques; Elsevier: Amsterdam, The Netherlands, 2013; ISBN 9780123822390.

24. Petsagkourakis, I.; Kim, N.; Tybrandt, K.; Zozoulenko, I.; Crispin, X. Poly(3,4-ethylenedioxythiophene): Chemical Synthesis, Transport Properties, and Thermoelectric Devices. Adv. Electron. Mater. 2019, 5, 1800918. [CrossRef]

25. Winther-Jensen, B.; Breiby, D.W.; West, K. Base inhibited oxidative polymerization of 3,4-ethylenedioxythiophene with iron(III)tosylate. Synth. Met. 2005, 152, 1-4. [CrossRef]

26. Kirchmeyer, S.; Reuter, K. Scientific importance, properties and growing applications of poly(3,4-ethylenedioxythiophene). $J$ Mater. Chem. 2005, 15, 2077-2088. [CrossRef]

27. Kim, D.; Zozoulenko, I. Why Is Pristine PEDOT Oxidized to 33\%? A Density Functional Theory Study of Oxidative Polymerization Mechanism. J. Phys. Chem. B 2019, 123, 5160-5167. [CrossRef] [PubMed]

28. Ha, Y.H.; Nikolov, N.; Pollack, S.K.; Mastrangelo, J.; Martin, B.D.; Shashidhar, R. Towards a transparent, highly conductive poly (3,4-ethylenedioxythiophene). Adv. Funct. Mater. 2004, 14, 615-622. [CrossRef]

29. Elschner, A.; Kirchmeyer, S.; Lövenich, W.; Merker, U.; Reuter, K. PEDOT: Principles and Applications of an Intrinsically Conductive Polymer; CRC Press: Boca Raton, FL, USA, 2010; ISBN 9781420069129.

30. Gueye, M.N.; Carella, A.; Massonnet, N.; Yvenou, E.; Brenet, S.; Faure-Vincent, J.; Pouget, S.; Rieutord, F.; Okuno, H.; Benayad, A.; et al. Structure and Dopant Engineering in PEDOT Thin Films: Practical Tools for a Dramatic Conductivity Enhancement. Chem. Mater. 2016, 28, 3462-3468. [CrossRef] 
31. Khan, Z.U.; Bubnova, O.; Jafari, M.J.; Brooke, R.; Liu, X.; Gabrielsson, R.; Ederth, T.; Evans, D.R.; Andreasen, J.W.; Fahlman, M.; et al. Acido-basic control of the thermoelectric properties of poly(3,4-ethylenedioxythiophene)tosylate (PEDOT-Tos) thin films. J. Mater. Chem. C 2015, 3, 10616-10623. [CrossRef] [PubMed]

32. Shi, W.; Yao, Q.; Qu, S.; Chen, H.; Zhang, T.; Chen, L. Micron-thick highly conductive PEDOT films synthesized via self-inhibited polymerization: Roles of anions. NPG Asia Mater. 2017, 9, e405. [CrossRef]

33. Kim, J.S.; Jang, W.; Wang, D.H. The investigation of the seebeck effect of the poly(3,4-ethylenedioxythiophene)-tosylate with the various concentrations of an oxidant. Polymers 2018, 11, 21. [CrossRef] [PubMed]

34. Cosnier, S.; Karyakin, A.; Cosnier, S. Electropolymerization; Cosnier, S., Karyakin, A., Eds.; Wiley-VCH Verlag GmbH \& Co. KGaA: Weinheim, Germany, 2010; ISBN 9783527630592.

35. Pettersson, L.A.A.; Carlsson, F.; Inganäs, O.; Arwin, H. Spectroscopic ellipsometry studies of the optical properties of doped poly(3,4-ethylenedioxythiophene): An anisotropic metal. Thin Solid Films 1998, 313-314, 356-361. [CrossRef]

36. Zotti, G.; Zecchin, S.; Schiavon, G.; Louwet, F.; Groenendaal, L.; Crispin, X.; Osikowicz, W.; Salaneck, W.; Fahlman, M. Electrochemical and XPS Studies toward the Role of Monomeric and Polymeric Sulfonate Counterions in the Synthesis, Composition, and Properties of Poly(3,4-ethylenedioxythiophene). Macromolecules 2003, 36, 3337-3344. [CrossRef]

37. Benoudjit, A.; Bader, M.M.; Wan Salim, W.W.A. Study of electropolymerized PEDOT:PSS transducers for application as electrochemical sensors in aqueous media. Sens. Bio-Sens. Res. 2018, 17, 18-24. [CrossRef]

38. Melato, A.I.; Mendonça, M.H.; Abrantes, L.M. Effect of the electropolymerisation conditions on the electrochemical, morphological and structural properties of PEDOTh films. J. Solid State Electrochem. 2009, 13, 417-426. [CrossRef]

39. Poverenov, E.; Li, M.; Bitler, A.; Bendikov, M. Major effect of electropolymerization solvent on morphology and electrochromic properties of PEDOT films. Chem. Mater. 2010, 22, 4019-4025. [CrossRef]

40. Cysewska, K.; Karczewski, J.; Jasiński, P. Influence of electropolymerization conditions on the morphological and electrical properties of PEDOT film. Electrochim. Acta 2015, 176, 156-161. [CrossRef]

41. Du, X.; Wang, Z. Effects of polymerization potential on the properties of electrosynthesized PEDOT films. Electrochim. Acta 2003, 48, 1713-1717. [CrossRef]

42. Castagnola, V.; Descamps, E.; Lecestre, A.; Dahan, L.; Remaud, J.; Nowak, L.G.; Bergaud, C. Parylene-based flexible neural probes with PEDOT coated surface for brain stimulation and recording. Biosens. Bioelectron. 2015, 67, 450-457. [CrossRef]

43. Aubert, P.H.; Groenendaal, L.; Louwet, F.; Lutsen, L.; Vanderzande, D.; Zotti, G. In situ conductivity measurements on polyethylenedioxythiophene derivatives with different counter ions. Synth. Met. 2002, 126, 193-198. [CrossRef]

44. Castagnola, V.; Bayon, C.; Descamps, E.; Bergaud, C. Morphology and conductivity of PEDOT layers produced by different electrochemical routes. Synth. Met. 2014, 189, 7-16. [CrossRef]

45. Sandoval-Rojas, A.P.; Cortés, M.T.; Hurtado, J. Electrochemical synthesis of poly(3,4-ethylenedioxythiophene) doped with a new bis(pyrazolyl)methane disulfonate and its behavior towards dopamine detection. J. Electroanal. Chem. 2019, 837, $200-207$. [CrossRef]

46. Kim, J.; Kim, E.; Won, Y.; Lee, H.; Suh, K. The preparation and characteristics of conductive poly(3,4-ethylenedioxythiophene) thin film by vapor-phase polymerization. Synth. Met. 2003, 139, 485-489. [CrossRef]

47. Winther-Jensen, B.; West, K. Vapor-phase polymerization of 3,4-ethylenedioxythiophene: A route to highly conducting polymer surface layers. Macromolecules 2004, 37, 4538-4543. [CrossRef]

48. Mueller, M.; Fabretto, M.; Evans, D.; Hojati-Talemi, P.; Gruber, C.; Murphy, P. Vacuum vapour phase polymerization of high conductivity PEDOT: Role of PEG-PPG-PEG, the origin of water, and choice of oxidant. Polymer 2012, 53, 2146-2151. [CrossRef]

49. Lawal, A.T.; Wallace, G.G. Vapour phase polymerisation of conducting and non-conducting polymers: A review. Talanta 2014, 119, 133-143. [CrossRef] [PubMed]

50. Chelawat, H.; Vaddiraju, S.; Gleason, K. Conformal, conducting poly(3,4-ethylenedioxythiophene) thin films deposited using bromine as the oxidant in a completely dry oxidative chemical vapor deposition process. Chem. Mater. 2010, 22, 2864-2868. [CrossRef]

51. Chen, X.; Zhu, C.; Jiang, F.; Liu, G.; Liu, C.; Jiang, Q.; Xu, J.; An, J.; Liu, P. Regulating monomer assembly to enhance PEDOT capacitance performance via different oxidants. J. Colloid Interface Sci. 2021, 601, 265-271. [CrossRef]

52. Zuber, K.; Fabretto, M.; Hall, C.; Murphy, P. Improved PEDOT conductivity via suppression of crystallite formation in Fe(III) tosylate during vapor phase polymerization. Macromol. Rapid Commun. 2008, 29, 1503-1508. [CrossRef]

53. Wang, W.; Xu, D.; Huang, A.; Yuan, H.; Xie, J.; Chen, X.; He, Y.; Zhang, T.; Shen, H. Controllable vapor phase polymerization of PEDOT films using imidazole as an inhibitor and their electrical and electrochromic properties. Synth. Met. 2020, $269,116523$. [CrossRef]

54. Lee, S.; Paine, D.C.; Gleason, K.K. Heavily doped poly(3,4-ethylenedioxythiophene) thin films with high carrier mobility deposited using oxidative CVD: Conductivity stability and carrier transport. Adv. Funct. Mater. 2014, 24, 7187-7196. [CrossRef]

55. Drewelow, G.; Wook Song, H.; Jiang, Z.T.; Lee, S. Factors controlling conductivity of PEDOT deposited using oxidative chemical vapor deposition. Appl. Surf. Sci. 2020, 501, 114105. [CrossRef]

56. Mirabedin, M.; Vergnes, H.; Caussé, N.; Vahlas, C.; Caussat, B. Liquid antimony pentachloride as oxidant for robust oxidative chemical vapor deposition of poly(3,4-ethylenedioxythiophene) films. Appl. Surf. Sci. 2021, 554, 149501. [CrossRef] 
57. Atanasov, S.E.; Losego, M.D.; Gong, B.; Sachet, E.; Maria, J.P.; Williams, P.S.; Parsons, G.N. Highly conductive and conformal poly(3,4-ethylenedioxythiophene) (PEDOT) thin films via oxidative molecular layer deposition. Chem. Mater. 2014, 26, 3471-3478. [CrossRef]

58. Moni, P.; Lau, J.; Mohr, A.C.; Lin, T.C.; Tolbert, S.H.; Dunn, B.; Gleason, K.K. Growth Temperature and Electrochemical Performance in Vapor-Deposited Poly(3,4-ethylenedioxythiophene) Thin Films for High-Rate Electrochemical Energy Storage. ACS Appl. Energy Mater. 2018, 1, 7093-7105. [CrossRef]

59. Mirabedin, M.; Vergnes, H.; Caussé, N.; Vahlas, C.; Caussat, B. An out of the box vision over oxidative chemical vapor deposition of PEDOT involving sublimed iron trichloride. Synth. Met. 2020, 266, 1-12. [CrossRef]

60. Wen, Y.; Xu, J. Scientific Importance of Water-Processable PEDOT-PSS and Preparation, Challenge and New Application in Sensors of Its Film Electrode: A Review. J. Polym. Sci. Part A Polym. Chem. 2017, 55, 1121-1150. [CrossRef]

61. Perinka, N.; Kim, C.H.; Kaplanova, M.; Bonnassieux, Y. Preparation and Characterization of Thin Conductive Polymer Films on the base of PEDOT:PSS by Ink-Jet Printing. Phys. Procedia 2013, 44, 120-129. [CrossRef]

62. Yuk, H.; Lu, B.; Lin, S.; Qu, K.; Xu, J.; Luo, J.; Zhao, X. 3D printing of conducting polymers. Nat. Commun. 2020, 11, 4-11. [CrossRef]

63. Horii, T.; Hikawa, H.; Katsunuma, M.; Okuzaki, H. Synthesis of highly conductive PEDOT:PSS and correlation with hierarchical structure. Polymer 2018, 140, 33-38. [CrossRef]

64. Gao, N.; Yu, J.; Tian, Q.; Shi, J.; Zhang, M.; Chen, S.; Zang, L. Application of pedot:Pss and its composites in electrochemical and electronic chemosensors. Chemosensors 2021, 9, 79. [CrossRef]

65. Shi, H.; Liu, C.; Jiang, Q.; Xu, J. Effective Approaches to Improve the Electrical Conductivity of PEDOT:PSS: A Review. Adv. Electron. Mater. 2015, 1, 1-16. [CrossRef]

66. Hofmann, A.I.; Katsigiannopoulos, D.; Mumtaz, M.; Petsagkourakis, I.; Pecastaings, G.; Fleury, G.; Schatz, C.; Pavlopoulou, E.; Brochon, C.; Hadziioannou, G.; et al. How to Choose Polyelectrolytes for Aqueous Dispersions of Conducting PEDOT Complexes. Macromolecules 2017, 50, 1959-1969. [CrossRef]

67. Mantione, D.; del Agua, I.; Sanchez-Sanchez, A.; Mecerreyes, D. Poly(3,4-ethylenedioxythiophene) (PEDOT) derivatives: Innovative conductive polymers for bioelectronics. Polymers 2017, 9, 354. [CrossRef] [PubMed]

68. Donahue, M.J.; Sanchez-Sanchez, A.; Inal, S.; Qu, J.; Owens, R.M.; Mecerreyes, D.; Malliaras, G.G.; Martin, D.C. Tailoring PEDOT properties for applications in bioelectronics. Mater. Sci. Eng. R Rep. 2020, 140, 100546. [CrossRef]

69. Casalini, S.; Dumitru, A.C.; Leonardi, F.; Bortolotti, C.A.; Herruzo, E.T.; Campana, A.; De Oliveira, R.F.; Cramer, T.; Garcia, R.; Biscarini, F. Multiscale sensing of antibody-antigen interactions by organic transistors and single-molecule force spectroscopy. ACS Nano 2015, 9, 5051-5062. [CrossRef] [PubMed]

70. Sassolas, A.; Blum, L.J.; Leca-Bouvier, B.D. Immobilization strategies to develop enzymatic biosensors. Biotechnol. Adv. 2012, 30, 489-511. [CrossRef] [PubMed]

71. Fenoy, G.E.; Marmisollé, W.A.; Azzaroni, O.; Knoll, W. Acetylcholine biosensor based on the electrochemical functionalization of graphene field-effect transistors. Biosens. Bioelectron. 2020, 148, 111796. [CrossRef] [PubMed]

72. Hackett, A.J.; Malmström, J.; Travas-Sejdic, J. Functionalization of conducting polymers for biointerface applications. Prog. Polym. Sci. 2017, 70, 18-33. [CrossRef]

73. Xia, Y.; Ouyang, J. Significant Different Conductivities of the Two Grades of Poly(3,4-ethylenedioxythiophene):Poly(styrenesulfonate), Clevios P and Clevios PH1000, Arising from Different Molecular Weights. ACS Appl. Mater. Interfaces 2012, 4, 4131-4140. [CrossRef]

74. Galán, T.; Prieto-Simón, B.; Alvira, M.; Eritja, R.; Götz, G.; Bäuerle, P.; Samitier, J. Label-free electrochemical DNA sensor using "click"-functionalized PEDOT electrodes. Biosens. Bioelectron. 2015, 74, 751-756. [CrossRef]

75. Bazaco, R.B.; Gómez, R.; Seoane, C.; Bäuerle, P.; Segura, J.L. Specific recognition of a nucleobase-functionalized poly(3,4ethylenedioxithiophene) (PEDOT) in aqueous media. Tetrahedron Lett. 2009, 50, 4154-4157. [CrossRef]

76. Dong, L.; Lu, B.; Duan, X.; Xu, J.; Hu, D.; Zhang, K.; Zhu, X.; Sun, H.; Ming, S.; Wang, Z.; et al. Novel chiral PEDOTs for selective recognition of 3,4-dihydroxyphenylalanine enantiomers: Synthesis and characterization. J. Polym. Sci. Part A Polym. Chem. 2015, 53, 2238-2251. [CrossRef]

77. Bhagwat, N.; Kiick, K.L.; Martin, D.C. Electrochemical deposition and characterization of carboxylic acid functionalized PEDOT copolymers. J. Mater. Res. 2014, 29, 2835-2844. [CrossRef]

78. Bhagwat, N.; Murray, R.E.; Shah, S.I.; Kiick, K.L.; Martin, D.C. Biofunctionalization of PEDOT films with laminin-derived peptides. Acta Biomater. 2016, 41, 235-246. [CrossRef] [PubMed]

79. Povlich, L.K.; Cho, J.C.; Leach, M.K.; Corey, J.M.; Kim, J.; Martin, D.C. Synthesis, copolymerization and peptide-modification of carboxylic acid-functionalized 3,4-ethylenedioxythiophene (EDOTacid) for neural electrode interfaces. Biochim. Biophys. Acta (BBA)-Gen. Subj. 2013, 1830, 4288-4293. [CrossRef]

80. Ouyang, L.; Kuo, C.C.; Farrell, B.; Pathak, S.; Wei, B.; Qu, J.; Martin, D.C. Poly[3,4-ethylene dioxythiophene (EDOT)-co-1,3,5-tri[2(3,4-ethylene dioxythienyl)]-benzene (EPh)] copolymers (PEDOT-co-EPh): Optical, electrochemical and mechanical properties. J. Mater. Chem. B 2015, 3, 5010-5020. [CrossRef]

81. Mantione, D.; Stavrinidou, E.; Pavlopoulou, E.; Istif, E.; Dufil, G.; Vallan, L.; Parker, D.; Brochon, C.; Cloutet, E.; Hadziioannou, G.; et al. Thiophene-based trimers for in vivo electronic functionalization of tissues. ACS Appl. Electron. Mater. 2020, 2, $4065-4071$. [CrossRef] 
82. Wei, B.; Ouyang, L.; Liu, J.; Martin, D.C. Post-polymerization functionalization of poly(3,4-propylenedioxythiophene) (PProDOT) via thiol-ene "click" chemistry. J. Mater. Chem. B 2015, 3, 5028-5034. [CrossRef] [PubMed]

83. Hai, W.; Goda, T.; Takeuchi, H.; Yamaoka, S.; Horiguchi, Y.; Matsumoto, A.; Miyahara, Y. Specific Recognition of Human Influenza Virus with PEDOT Bearing Sialic Acid-Terminated Trisaccharides. ACS Appl. Mater. Interfaces 2017, 9, 14162-14170. [CrossRef]

84. Daprà, J.; Lauridsen, L.H.; Nielsen, A.T.; Rozlosnik, N. Comparative study on aptamers as recognition elements for antibiotics in a label-free all-polymer biosensor. Biosens. Bioelectron. 2013, 43, 315-320. [CrossRef] [PubMed]

85. Jimison, L.H.; Hama, A.; Strakosas, X.; Armel, V.; Khodagholy, D.; Ismailova, E.; Malliaras, G.G.; Winther-Jensen, B.; Owens, R.M. PEDOT:TOS with PEG: A biofunctional surface with improved electronic characteristics. J. Mater. Chem. 2012, 22, 19498-19505. [CrossRef]

86. Fabretto, M.; Jariego-Moncunill, C.; Autere, J.P.; Michelmore, A.; Short, R.D.; Murphy, P. High conductivity PEDOT resulting from glycol/oxidant complex and glycol/polymer intercalation during vacuum vapour phase polymerisation. Polymer 2011, 52, 1725-1730. [CrossRef]

87. Martinelli, A.; D’Ilario, L.; Francolini, I.; Piozzi, A.; Pizzi, E. Partially sulfonated ethylene-vinyl alcohol copolymer as new substrate for 3,4-ethylenedioxythiophene vapor phase polymerization. J. Polym. Sci. Part B Polym. Phys. 2014, 52, 1203-1210. [CrossRef]

88. Bongo, M.; Winther-Jensen, O.; Himmelberger, S.; Strakosas, X.; Ramuz, M.; Hama, A.; Stavrinidou, E.; Malliaras, G.G.; Salleo, A.; Winther-Jensen, B.; et al. PEDOT:gelatin composites mediate brain endothelial cell adhesion. J. Mater. Chem. B 2013, 1, 3860-3867. [CrossRef] [PubMed]

89. Sappia, L.D.; Piccinini, E.; Marmisollé, W.; Santilli, N.; Maza, E.; Moya, S.; Battaglini, F.; Madrid, R.E.; Azzaroni, O. Integration of Biorecognition Elements on PEDOT Platforms through Supramolecular Interactions. Adv. Mater. Interfaces 2017, 4, 1-11. [CrossRef]

90. Sappia, L.D.; Piccinini, E.; von Binderling, C.; Knoll, W.; Marmisollé, W.; Azzaroni, O. PEDOT-polyamine composite films for bioelectrochemical platforms-flexible and easy to derivatize. Mater. Sci. Eng. C 2020, 109, 110575. [CrossRef] [PubMed]

91. Fenoy, G.E.; Bilderling, C.; Knoll, W.; Azzaroni, O.; Marmisollé, W.A. PEDOT:Tosylate-Polyamine-Based Organic Electrochemical Transistors for High-Performance Bioelectronics. Adv. Electron. Mater. 2021, 7, 2100059. [CrossRef]

92. Zamora-Sequeira, R.; Ardao, I.; Starbird, R.; García-González, C.A. Conductive nanostructured materials based on poly-(3,4ethylenedioxythiophene) (PEDOT) and starch/K-carrageenan for biomedical applications. Carbohydr. Polym. 2018, 189, 304-312. [CrossRef]

93. Harman, D.G.; Gorkin, R.; Stevens, L.; Thompson, B.; Wagner, K.; Weng, B.; Chung, J.H.Y.; In Het Panhuis, M.; Wallace, G.G. Poly(3,4-ethylenedioxythiophene):dextran sulfate (PEDOT:DS)—A highly processable conductive organic biopolymer. Acta Biomater. 2015, 14, 33-42. [CrossRef]

94. Xiao, Y.; Li, C.M.; Wang, S.; Shi, J.; Ooi, C.P. Incorporation of collagen in poly(3,4-ethylenedioxythiophene) for a bifunctional film with high bio- and electrochemical activity. J. Biomed. Mater. Res. Part A 2010, 92, 766-772. [CrossRef] [PubMed]

95. Vara, H.; Collazos-Castro, J.E. Biofunctionalized Conducting Polymer/Carbon Microfiber Electrodes for Ultrasensitive Neural Recordings. ACS Appl. Mater. Interfaces 2015, 7, 27016-27026. [CrossRef]

96. Polino, G.; Lubrano, C.; Scognamiglio, P.; Mollo, V.; De Martino, S.; Ciccone, G.; Matino, L.; Langella, A.; Netti, P.; Di Carlo, A.; et al. Synthesis and characterization of PEDOT-PEGDA blends for bioelectronic applications: Surface properties and effects on cell morphology. Flex. Print. Electron. 2020, 5, 014012. [CrossRef]

97. Abedi, A.; Hasanzadeh, M.; Tayebi, L. Conductive nanofibrous Chitosan/PEDOT:PSS tissue engineering scaffolds. Mater. Chem. Phys. 2019, 237. [CrossRef]

98. Kumar, S.; Rai, P.; Sharma, J.G.; Sharma, A.; Malhotra, B.D. PEDOT:PSS/PVA-Nanofibers-Decorated Conducting Paper for Cancer Diagnostics. Adv. Mater. Technol. 2016, 1, 1-8. [CrossRef]

99. Strakosas, X.; Sessolo, M.; Hama, A.; Rivnay, J.; Stavrinidou, E.; Malliaras, G.G.; Owens, R.M. A facile biofunctionalisation route for solution processable conducting polymer devices. J. Mater. Chem. B 2014, 2, 2537-2545. [CrossRef] [PubMed]

100. He, R.X.; Zhang, M.; Tan, F.; Leung, P.H.M.; Zhao, X.Z.; Chan, H.L.W.; Yang, M.; Yan, F. Detection of bacteria with organic electrochemical transistors. J. Mater. Chem. 2012, 22, 22072-22076. [CrossRef]

101. Ferlauto, L.; D’Angelo, A.N.; Vagni, P.; Leccardi, M.J.I.A.; Mor, F.M.; Cuttaz, E.A.; Heuschkel, M.O.; Stoppini, L.; Ghezzi, D. Development and characterization of PEDOT:PSS/alginate soft microelectrodes for application in neuroprosthetics. Front. Neurosci. 2018, 12, 1-10. [CrossRef] [PubMed]

102. Collazos-Castro, J.E.; Polo, J.L.; Hernández-Labrado, G.R.; Padial-Cañete, V.; García-Rama, C. Bioelectrochemical control of neural cell development on conducting polymers. Biomaterials 2010, 31, 9244-9255. [CrossRef]

103. Fenoy, G.E.; Maza, E.; Zelaya, E.; Marmisollé, W.A.; Azzaroni, O. Layer-by-layer assemblies of highly connected polyelectrolyte capped-Pt nanoparticles for electrocatalysis of hydrogen evolution reaction. Appl. Surf. Sci. 2017, 416, 24-32. [CrossRef]

104. Decher, G.; Hong, J.D.; Schmitt, J. Buildup of ultrathin multilayer films by a self-assembly process: III. Consecutively alternating adsorption of anionic and cationic polyelectrolytes on charged surfaces. Thin Solid Films 1992, 831-835. [CrossRef]

105. Iler, R.K. Multilayers of colloidal particles. J. Colloid Interface Sci. 1966, 21, 569-594. [CrossRef]

106. Richardson, J.J.; Cui, J.; Björnmalm, M.; Braunger, J.A.; Ejima, H.; Caruso, F. Innovation in Layer-by-Layer Assembly. Chem. Rev. 2016, 116, 14828-14867. [CrossRef] [PubMed] 
107. Rydzek, G.; Ji, Q.; Li, M.; Schaaf, P.; Hill, J.P.; Boulmedais, F.; Ariga, K. Electrochemical nanoarchitectonics and layer-by-layer assembly: From basics to future. Nano Today 2015, 10, 138-167. [CrossRef]

108. Pappa, A.M.; Inal, S.; Roy, K.; Zhang, Y.; Pitsalidis, C.; Hama, A.; Pas, J.; Malliaras, G.G.; Owens, R.M. Polyelectrolyte Layerby-Layer Assembly on Organic Electrochemical Transistors. ACS Appl. Mater. Interfaces 2017, 9, 10427-10434. [CrossRef] [PubMed]

109. Hardy, J.G.; Li, H.; Chow, J.K.; Geissler, S.A.; Mcelroy, A.B.; Nguy, L.; Hernandez, D.S.; Schmidt, C.E. Conducting polymer-based multilayer films for instructive biomaterial coatings Dip coating direction Weakly aligned broblasts on multilayer lms: Glassconducting polymer-chitosan-gelatin Passage of a DC current through the lm enhances cell alignment DC cu. Futur. Sci. OA 2015, 1,79 .

110. David, M.; Barsan, M.M.; Brett, C.M.A.; Florescu, M. Improved glucose label-free biosensor with layer-by-layer architecture and conducting polymer poly(3,4-ethylenedioxythiophene). Sens. Actuators B Chem. 2018, 255, 3227-3234. [CrossRef]

111. Daikuzono, C.M.; Dantas, C.A.R.; Volpati, D.; Constantino, C.J.L.; Piazzetta, M.H.O.; Gobbi, A.L.; Taylor, D.M.; Oliveira, O.N.; Riul, A. Microfluidic electronic tongue. Sens. Actuators B Chem. 2015, 207, 1129-1135. [CrossRef]

112. Khodagholy, D.; Rivnay, J.; Sessolo, M.; Gurfinkel, M.; Leleux, P.; Jimison, L.H.; Stavrinidou, E.; Herve, T.; Sanaur, S.; Owens, R.M.; et al. High transconductance organic electrochemical transistors. Nat. Commun. 2013, 4, 1-6. [CrossRef]

113. Liao, J.; Si, H.; Zhang, X.; Lin, S. Functional sensing interfaces of PEDOT:PSS organic electrochemical transistors for chemical and biological sensors: A mini review. Sensors 2019, 19, 218. [CrossRef]

114. Hempel, F.; Law, J.K.Y.; Nguyen, T.C.; Lanche, R.; Susloparova, A.; Vu, X.T.; Ingebrandt, S. PEDOT:PSS organic electrochemical transistors for electrical cell-substrate impedance sensing down to single cells. Biosens. Bioelectron. 2021, 180, 113101. [CrossRef] [PubMed]

115. Rivnay, J.; Leleux, P.; Ferro, M.; Sessolo, M.; Williamson, A.; Koutsouras, D.A.; Khodagholy, D.; Ramuz, M.; Strakosas, X.; Owens, R.M.; et al. High-performance transistors for bioelectronics through tuning of channel thickness. Sci. Adv. 2015, 1, e1400251. [CrossRef] [PubMed]

116. Liang, Y.; Ernst, M.; Brings, F.; Kireev, D.; Maybeck, V.; Offenhäusser, A.; Mayer, D. High Performance Flexible Organic Electrochemical Transistors for Monitoring Cardiac Action Potential. Adv. Healthc. Mater. 2018, 7, 1800304. [CrossRef] [PubMed]

117. Inal, S.; Malliaras, G.G.; Rivnay, J. Benchmarking organic mixed conductors for transistors. Nat. Commun. 2017, 8, 1-6. [CrossRef]

118. Cea, C.; Spyropoulos, G.D.; Jastrzebska-Perfect, P.; Ferrero, J.J.; Gelinas, J.N.; Khodagholy, D. Enhancement-mode ion-based transistor as a comprehensive interface and real-time processing unit for in vivo electrophysiology. Nat. Mater. 2020, 19, 679-686. [CrossRef]

119. Hai, W.; Goda, T.; Takeuchi, H.; Yamaoka, S.; Horiguchi, Y.; Matsumoto, A.; Miyahara, Y. Human influenza virus detection using sialyllactose-functionalized organic electrochemical transistors. Sens. Actuators B Chem. 2018, 260, 635-641. [CrossRef]

120. Chakrabarti, M.H.; Low, C.T.J.; Brandon, N.P.; Yufit, V.; Hashim, M.A.; Irfan, M.F.; Akhtar, J.; Ruiz-Trejo, E.; Hussain, M.A. Progress in the electrochemical modification of graphene-based materials and their applications. Electrochim. Acta 2013, 107, 425-440. [CrossRef]

121. Buth, F.; Donner, A.; Sachsenhauser, M.; Stutzmann, M.; Garrido, J.A. Biofunctional electrolyte-gated organic field-effect transistors. Adv. Mater. 2012, 24, 4511-4517. [CrossRef]

122. Wustoni, S.; Hidalgo, T.C.; Hama, A.; Ohayon, D.; Savva, A.; Wei, N.; Wehbe, N.; Inal, S. In Situ Electrochemical Synthesis of a Conducting Polymer Composite for Multimetabolite Sensing. Adv. Mater. Technol. 2020, 5, 1-9. [CrossRef]

123. Carli, S.; Di Lauro, M.; Bianchi, M.; Murgia, M.; De Salvo, A.; Prato, M.; Fadiga, L.; Biscarini, F. Water-Based PEDOT:Nafion Dispersion for Organic Bioelectronics. ACS Appl. Mater. Interfaces 2020, 12, 29807-29817. [CrossRef]

124. Ronkainen, N.J.; Halsall, H.B.; Heineman, W.R. Electrochemical biosensors. Chem. Soc. Rev. 2010, 39, 1747. [CrossRef]

125. Iost, R.M.; Crespilho, F.N. Layer-by-layer self-assembly and electrochemistry: Applications in biosensing and bioelectronics. Biosens. Bioelectron. 2012, 31, 1-10. [CrossRef] [PubMed]

126. Dhand, C.; Das, M.; Datta, M.; Malhotra, B.D. Recent advances in polyaniline based biosensors. Biosens. Bioelectron. 2011, 26, 2811-2821. [CrossRef] [PubMed]

127. Wolfart, F.; Hryniewicz, B.M.; Góes, M.S.; Corrêa, C.M.; Torresi, R.; Minadeo, M.A.O.S.; Córdoba de Torresi, S.I.; Oliveira, R.D.; Marchesi, L.F.; Vidotti, M. Conducting polymers revisited: Applications in energy, electrochromism and molecular recognition. J. Solid State Electrochem. 2017, 21, 2489-2515. [CrossRef]

128. Scouten, W.H.; Luong, J.H.T.; Stephen Brown, R. Enzyme or protein immobilization techniques for applications in biosensor design. Trends Biotechnol. 1995, 13, 178-185. [CrossRef]

129. Meng, L.; Turner, A.P.F.; Mak, W.C. Tunable 3D nanofibrous and bio-functionalised PEDOT network explored as a conducting polymer-based biosensor. Biosens. Bioelectron. 2020, 159, 112181. [CrossRef]

130. Jayathilaka, W.A.D.M.; Qi, K.; Qin, Y.; Chinnappan, A.; Serrano-García, W.; Baskar, C.; Wang, H.; He, J.; Cui, S.; Thomas, S.W.; et al. Significance of Nanomaterials in Wearables: A Review on Wearable Actuators and Sensors. Adv. Mater. 2019, 31, 1-21. [CrossRef] [PubMed]

131. Yang, Y.; Gao, W. Wearable and flexible electronics for continuous molecular monitoring. Chem. Soc. Rev. 2019, 48, 1465-1491. [CrossRef] 
132. Scotto, J.; Piccinini, E.; von Bilderling, C.; Coria-Oriundo, L.L.; Battaglini, F.; Knoll, W.; Marmisolle, W.A.; Azzaroni, O. Flexible conducting platforms based on PEDOT and graphite nanosheets for electrochemical biosensing applications. Appl. Surf. Sci. 2020, 525, 146440. [CrossRef]

133. Ha, J.; Chung, S.; Pei, M.; Cho, K.; Yang, H.; Hong, Y. One-Step Interface Engineering for All-Inkjet-Printed, All-Organic Components in Transparent, Flexible Transistors and Inverters: Polymer Binding. ACS Appl. Mater. Interfaces 2017, 9, 8819-8829. [CrossRef]

134. Yu, Y.; Nyein, H.Y.Y.; Gao, W.; Javey, A. Flexible Electrochemical Bioelectronics: The Rise of In Situ Bioanalysis. Adv. Mater. 2020, 32, 1-25. [CrossRef] [PubMed]

135. Xu, M.; Yadavalli, V.K. Flexible Biosensors for the Impedimetric Detection of Protein Targets Using Silk-Conductive Polymer Biocomposites. ACS Sens. 2019, 4, 1040-1047. [CrossRef] [PubMed]

136. Pal, R.K.; Farghaly, A.A.; Wang, C.; Collinson, M.M.; Kundu, S.C.; Yadavalli, V.K. Conducting polymer-silk biocomposites for flexible and biodegradable electrochemical sensors. Biosens. Bioelectron. 2016, 81, 294-302. [CrossRef]

137. Del Agua, I.; Mantione, D.; Ismailov, U.; Sanchez-Sanchez, A.; Aramburu, N.; Malliaras, G.G.; Mecerreyes, D.; Ismailova, E. DVS-Crosslinked PEDOT:PSS Free-Standing and Textile Electrodes toward Wearable Health Monitoring. Adv. Mater. Technol. 2018, 3, 1-8. [CrossRef]

138. Covey, E.; Carter, M. Basic Electrophysiological Methods; Oxford University Press: Oxford, UK, 2015; ISBN 9780199939862.

139. Walz, W. Electrophysiological Recording Techniques; Neuromethods; Vertes, R.P., Stackman, R.W., Eds.; Humana Press: Totowa, NJ, USA, 2011; Volume 54, ISBN 978-1-60327-201-8.

140. Liang, Y.; Offenhäusser, A.; Ingebrandt, S.; Mayer, D. PEDOT:PSS-Based Bioelectronic Devices for Recording and Modulation of Electrophysiological and Biochemical Cell Signals. Adv. Healthc. Mater. 2021, 1-24. [CrossRef]

141. Dijk, G.; Rutz, A.L.; Malliaras, G.G. Stability of PEDOT:PSS-Coated Gold Electrodes in Cell Culture Conditions. Adv. Mater. Technol. 2020, 5. [CrossRef]

142. Jones, P.D.; Moskalyuk, A.; Barthold, C.; Gutöhrlein, K.; Heusel, G.; Schröppel, B.; Samba, R.; Giugliano, M. Low-Impedance 3D PEDOT:PSS Ultramicroelectrodes. Front. Neurosci. 2020, 14, 1-12. [CrossRef] [PubMed]

143. Taccola, S.; Poliziani, A.; Santonocito, D.; Mondini, A.; Denk, C.; Ide, A.N.; Oberparleiter, M.; Greco, F.; Mattoli, V. Toward the use of temporary tattoo electrodes for impedancemetric respiration monitoring and other electrophysiological recordings on skin. Sensors 2021, 21, 1197. [CrossRef]

144. Ferrari, L.M.; Sudha, S.; Tarantino, S.; Esposti, R.; Bolzoni, F.; Cavallari, P.; Cipriani, C.; Mattoli, V.; Greco, F. Ultraconformable Temporary Tattoo Electrodes for Electrophysiology. Adv. Sci. 2018, 5, 1-11. [CrossRef]

145. Velasco-Bosom, S.; Karam, N.; Carnicer-Lombarte, A.; Gurke, J.; Casado, N.; Tomé, L.C.; Mecerreyes, D.; Malliaras, G.G. Conducting Polymer-Ionic Liquid Electrode Arrays for High-Density Surface Electromyography. Adv. Healthc. Mater. 2021, 2100374. [CrossRef]

146. Carli, S.; Bianchi, M.; Zucchini, E.; Di Lauro, M.; Prato, M.; Murgia, M.; Fadiga, L.; Biscarini, F. Electrodeposited PEDOT:Nafion Composite for Neural Recording and Stimulation. Adv. Healthc. Mater. 2019, 8, 1-10. [CrossRef] [PubMed]

147. Vreeland, R.F.; Atcherley, C.W.; Russell, W.S.; Xie, J.Y.; Lu, D.; Laude, N.D.; Porreca, F.; Heien, M.L. Biocompatible PEDOT:Nafion composite electrode coatings for selective detection of neurotransmitters in vivo. Anal. Chem. 2015, 87, 2600-2607. [CrossRef]

148. Paulsen, B.D.; Tybrandt, K.; Stavrinidou, E.; Rivnay, J. Organic mixed ionic-electronic conductors. Nat. Mater. 2020, 19, 13-26. [CrossRef] [PubMed]

149. Fahlman, M.; Fabiano, S.; Gueskine, V.; Simon, D.; Berggren, M.; Crispin, X. Interfaces in organic electronics. Nat. Rev. Mater. 2019, 4, 627-650. [CrossRef]

150. Marzocchi, M.; Gualandi, I.; Calienni, M.; Zironi, I.; Scavetta, E.; Castellani, G.; Fraboni, B. Physical and Electrochemical Properties of PEDOT:PSS as a Tool for Controlling Cell Growth. ACS Appl. Mater. Interfaces 2015, 7, 17993-18003. [CrossRef]

151. Hardy, J.G.; Lee, J.Y.; Schmidt, C.E. Biomimetic conducting polymer-based tissue scaffolds. Curr. Opin. Biotechnol. 2013, 24, 847-854. [CrossRef]

152. Svennersten, K.; Bolin, M.H.; Jager, E.W.H.; Berggren, M.; Richter-Dahlfors, A. Electrochemical modulation of epithelia formation using conducting polymers. Biomaterials 2009, 30, 6257-6264. [CrossRef]

153. Maione, S.; Gil, A.M.; Fabregat, G.; Del Valle, L.J.; Triguero, J.; Laurent, A.; Jacquemin, D.; Estrany, F.; Jiménez, A.I.; Zanuy, D.; et al. Electroactive polymer-peptide conjugates for adhesive biointerfaces. Biomater. Sci. 2015, 3, 1395-1405. [CrossRef]

154. Sekine, J.; Luo, S.C.; Wang, S.; Zhu, B.; Tseng, H.R.; Yu, H.H. Functionalized conducting polymer nanodots for enhanced cell capturing: The synergistic effect of capture agents and nanostructures. Adv. Mater. 2011, 23, 4788-4792. [CrossRef]

155. Someya, T.; Bao, Z.; Malliaras, G.G. The rise of plastic bioelectronics. Nature 2016, 540, 379-385. [CrossRef]

156. Huang, L.; Yang, X.; Deng, L.; Ying, D.; Lu, A.; Zhang, L.; Yu, A.; Duan, B. Biocompatible Chitin Hydrogel Incorporated with PEDOT Nanoparticles for Peripheral Nerve Repair. ACS Appl. Mater. Interfaces 2021. [CrossRef]

157. Xu, Y.; Yang, X.; Thomas, A.K.; Patsis, P.A.; Kurth, T.; Kräter, M.; Eckert, K.; Bornhäuser, M.; Zhang, Y. Noncovalently Assembled Electroconductive Hydrogel. ACS Appl. Mater. Interfaces 2018, 10, 14418-14425. [CrossRef] [PubMed]

158. Spencer, A.R.; Primbetova, A.; Koppes, A.N.; Koppes, R.A.; Fenniri, H.; Annabi, N. Electroconductive Gelatin MethacryloylPEDOT:PSS Composite Hydrogels: Design, Synthesis, and Properties. ACS Biomater. Sci. Eng. 2018, 4, 1558-1567. [CrossRef] [PubMed] 